\title{
SHP-2 and canonical PD-1: SHP-2 axis regulate myeloid cell differentiation, anti-tumor responses and innate immune memory
}

Vassiliki Boussiotis ( $\sim$ vboussio@bidmc.harvard.edu)

Beth Israel Deaconess Medical Center https://orcid.org/0000-0002-3963-1518

Anthos Christofides

Beth Israel Deaconess Medical Center

Xanthi-Lida Katopodi

Beth Israel Deaconess Medical Center

Carol Cao

Beth Israel Deaconess Medical Center

Halil-Ibrahim Aksoylar

Beth Israel Deaconess Medical Center https://orcid.org/0000-0002-0527-6124

Mohamed Mahmoud

Beth Israel Deaconess Medical Center

\section{Laura Strauss}

Humanitas Clinical and Research Center, Via Manzoni 56, 20089 Rozzano, Milan, Italy

Natalia Tijaro-Ovalle

Beth Israel Deaconess Medical Center

Rinku Pal

Beth Israel Deaconess Medical Center

Louis Boon

Polpharma Biologics

John Asara

Harvard Medical School

loannis Vlachos

Beth Israel Deaconess Medical Center

Nikolaos Patsoukis

Beth Israel Deaconess Medical Center, Harvard Medical School

\section{Article}

Keywords: 
Posted Date: January 7th, 2022

DOI: https://doi.org/10.21203/rs.3.rs-1198158/v1

License: (c) (i) This work is licensed under a Creative Commons Attribution 4.0 International License. Read Full License 


\section{SHP-2 and canonical PD-1: SHP-2 axis regulate myeloid cell differentiation, anti-tumor responses and innate immune memory}

Anthos Christofides ${ }^{1,2,3}$, Xanthi-Lida Katopodi ${ }^{4,5}$, Carol Cao ${ }^{1,2,6}$, Ibrahim Aksoylar ${ }^{1,2,3}$, Mohamed AA Mahmoud $^{1,2, \#}$, Laura Strauss ${ }^{1,2,3^{\wedge}}$, Natalia M. Tijaro-Ovalle ${ }^{1,2 \&}$, Rinku Pal ${ }^{1,2,3}$, Louis Boon ${ }^{7}$, John Asara ${ }^{3}$, Ioannis S. Vlachos ${ }^{4,5,8,9}$, Nikolaos Patsoukis ${ }^{1,2,3}$, Vassiliki A. Boussiotis ${ }^{1,2,3}$

${ }^{1}$ Division of Hematology-Oncology; ${ }^{2}$ Department of Medicine; ${ }^{3}$ Cancer Center, Beth Israel Deaconess Medical Center; ${ }^{4}$ Department of Pathology, Beth Israel Deaconess Medical Center, Harvard Medical School, Boston, MA 02215; ${ }^{5}$ Harvard Medical School Initiative for RNA Medicine, Beth Israel Deaconess Medical Center, Boston, MA 02215, ${ }^{6}$ Harvard College, Cambridge, MA 02138; ${ }^{7}$ Polpharma Biologics, $3584 \mathrm{CM}$, Utrecht, The Netherlands. ${ }^{8}$ Cancer Research Institute, Beth Israel Deaconess Medical Center, Boston, MA 02215; ${ }^{9}$ Broad Institute of MIT and Harvard, Cambridge, MA 02142.

${ }^{\#}$ Current affiliation: Heidelberg University, German Cancer Research Center (DKFZ)

${ }^{\wedge}$ Current affiliation: EMD-Serono RND Institute, ImmunoPharmacology ImmunoOncology TIP, EMD Serono Billerica MA

${ }^{\&}$ Current affiliation: Department of Medicine, Yale University, Hew Haven Connecticut, CT 06510

*Correspondance :

Vassiliki A. Boussiotis, MD, PhD.

Beth Israel Deaconess Medical Center

330 Brookline Avenue

Dana 513

Boston MA 02215

Phone: 617-667-8563

FAX: 617-667-9922

Email:vboussio@bidmc.harvard.edu

One sentence summary: SHP-2 ablation induces inflammatory differentiation of myeloid cells and antitumor immunity 


\section{Abstract}

PD-1 checkpoint inhibitor induces T cell inactivation by recruiting SHP-2. However, T cell-specific SHP2-deficient mice do not have improved anti-tumor immunity. We generated mice with conditional targeting of the Ptpn11 gene (encoding for Shp-2) in $\mathrm{T}$ cells $\left(\mathrm{Shp} 2^{\mathrm{f} / \mathrm{L}} \mathrm{LckCre}\right)$ or myeloid cells (Shp2 ${ }^{\mathrm{f} / \mathrm{L}} \mathrm{Ly} \mathrm{MCre}$ ), and found that Shp $2^{\mathrm{ff}}$ LysMCre mice had diminished tumor growth. As determined by RNA-seq, this was paralleled by the presence of inflammatory neutrophils and tumor-associated macrophages (TAMs) with molecular signatures of enhanced differentiation, phagocytosis and antigenprocessing and presentation. SHP-2 deficient TAMs also had increased monocyte and dendritic cell (DC) specification transcription factors, chemokine and cytokine production, and expression of immunostimulatory molecules that promote $\mathrm{T}$ cell recruitment and activation. Monocytes from tumorbearing $\operatorname{Shp} 2{ }^{\mathrm{ff} / \mathrm{L}} \mathrm{L}$ sMCre mice suppressed tumor growth after transfer to naïve recipients indicating development of innate immune memory. In bone marrow myelocytes, GM-CSF, induced PD-1 expression, phosphorylation and interaction with SHP-2, the Src family kinase Lyn, and GM-CSF receptor beta chain, indicating that the PD-1:SHP-2 axis targets a key pathway of myelocyte differentiation. In contrast, SHP-2 deletion or antibody-mediated blockade of the PD-1:PD-L1 pathway enhanced phosphorylation of the transcription factors HOXA10 and IRF8 that regulate myeloid differentiation and monocytic/moDC lineage commitment, respectively. Thus, SHP-2 and the PD-1:SHP-2 axis pose a signaling restrain to myeloid differentiation and monocyte lineage commitment resulting in a myeloid landscape that suppresses anti-tumor immunity. 


\section{Introduction}

The immune system plays an important protective function against tumor development and progression ${ }^{1}$. To escape immunosurveillance, cancer cells have developed mechanisms that mask their immunogenic features, such as expression of ligands for inhibitory receptors that directly inhibit $\mathrm{T}$ cell responses by engaging immune inhibitory receptors such as programmed death-1 (PD-1) ${ }^{2}$. Tumors also alter myeloid cells, which constitute a significant cellular fraction of the microenvironment and can employ multiple mechanisms to actively suppress anti-tumor $\mathrm{T}$ cell responses ${ }^{3}$. Recent studies have shown that tumor-infiltrating myeloid cells also include immunostimulatory subsets ${ }^{4-7}$. Immunostimulatory myeloid cells include tumor-associated macrophages (TAMs) producing proinflammatory cytokines, conventionally named M1-like macrophages, as well as type 1 dendritic cells (DC1s). Conversely, immunosuppressive myeloid cells include conventional M2-like TAMs, as well as immature myeloid cells generated during cancer-driven emergency myelopoiesis from bone marrow common myeloid progenitors (CMPs) and granulocyte/macrophage progenitors (GMPs), defined as myeloid-derived suppressor cells $(\mathrm{MDSCs})^{8}$. MDSCs negatively correlate with anti-tumor immune responses and compromise the outcome of chemotherapy, cancer vaccines and checkpoint immunotherapy 9-11. Thus, depletion of immunosuppressive tumor infiltrating myeloid cells, conversion to immunostimulatory effector myeloid cells or blockade of their functions, might improve the outcome of chemoimmunotherapy ${ }^{7,12-15}$.

PD-1 is a $\mathrm{T}$ cell inhibitor and the most extensively exploited therapeutic target of cancer immunotherapy ${ }^{16}$. PD-1-mediated T cell inactivation is attributed to the function of SHP-2 phosphatase, which is activated by recruitment to PD-1 cytoplasmic tail ${ }^{17-20}$. Based on these established data, it was expected that SHP-2 ablation would abrogate the inhibitory pathway activated downstream of PD-1 receptor in T cells, and mice with SHP-2 ablation in T cells would neither be subjected to PD-1-mediated inhibition of anti-tumor immunity nor would they be benefitted from treatment with anti-PD-1 antibody. 
However, it was found that T cell-specific SHP-2 deletion did not improve anti-tumor immunity and did not alter responses to anti-PD-1 treatment ${ }^{21}$, but might rather have a detrimental effect in melanoma progression and metastasis ${ }^{22}$. PD-1 is also expressed in common myeloid progenitors (CMPs) and granulocyte/macrophage progenitors (GMPs), which accumulate during cancer-driven emergency myelopoiesis and give rise to immunosuppressive MDSC and TAMs. In tumor-bearing mice with myeloid-specific PD-1 ablation, accumulation of MDSCs is prevented, while output of differentiated effector myeloid cells with monocytic lineage dominance is increased ${ }^{23}$. It is currently unknown whether a canonical PD-1:SHP-2 axis is operative in myeloid cells.

Temporal activation of SHP-2 is critical for myeloid cell fate ${ }^{24}$. Gain of function SHP-2 mutations, resulting in constitutive phosphatase activation, prevent myeloid differentiation and lead to the accumulation of immature myelocytes and development of leukemia ${ }^{25}$. HOXA proteins, members of the HOX family of transcription factors involved in normal hematopoiesis ${ }^{26}$, are targets of SHP-2 ${ }^{27}$ HOXA genes are maximally expressed in committed myeloid progenitors and are critical for normal myeloid development, whereas their aberrations are associated with leukemia ${ }^{28,29}$. During growth factor-induced myelopoiesis, tyrosine phosphorylation of HOXA10 in two tyrosine residues in the HOXA10 homeodomain decreases its binding affinity for target gene promoters. This abrogates HOXA10-induced transcriptional repression and differentiation proceeds ${ }^{30}$. HOXA10 is a substrate of activated SHP-2, which dephosphorylates HOXA and prevents derepressing of HOXA target genes and myeloid cell differentiation ${ }^{27,31}$. In addition, SHP-2 regulates the function of IFN regulatory factor (IRF)-8, a transcription factor that has a decisive role in myeloid progenitor differentiation toward monocytes and DCs, while limiting granulocyte development ${ }^{32,33}$. Failure of IRF8 expression or function leads to the generation of MDSCs ${ }^{34}$. IRF8 phosphorylation on Tyr-95, localized in the conserved IRF domain, is mandatory for its nuclear translocation and initiation of gene transcription ${ }^{35}$. The cytoplasmic-nuclear 
shuttling of IRF8 is regulated by SHP-2, which dephosphorylates IRF8 and prevents its nuclear localization and transcriptional activation ${ }^{35,36}$.

In the present study we examined how SHP-2 regulates the output of cancer-driven emergency myelopoiesis and investigated whether a canonical PD-1:SHP-2 axis is operative in myeloid cells. We used mice with conditional targeting of the Ptpn11 gene (encoding for SHP-2) that leads to significant

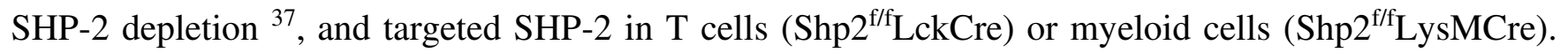
Although no difference in tumor growth was observed between Shp2 $2^{\mathrm{f} / \mathrm{L}}$ LckCre and control mice, Shp2 $2^{\mathrm{f} / \mathrm{L}}$ LysMCre mice had significantly reduced tumor growth that was not further decreased by anti-PD1 treatment. At a cellular level, myeloid-specific SHP-2 targeting skewed the myeloid cell fate in tumorbearing mice from MDSCs and converted them to differentiated myeloid cells that did not induce immunosuppression. As determined by RNA-seq, this was paralleled by the presence of inflammatory neutrophils and TAMs with molecular signatures of enhanced differentiation, phagocytosis, and antigen processing and presentation. SHP-2 deficient TAMs had increased expression of the monocyte specification transcription factors IRF8, Klf4 and Zeb2, enhanced molecular features of DC-producing monocytes (moDCs), chemokine and cytokine production, and expression of immunostimulatory molecules that promoted $\mathrm{T}$ cell recruitment and activation. Moreover, monocytes from tumor-bearing Shp2 $2^{\mathrm{f} / \mathrm{L}}$ LysMCre mice suppressed tumor growth after transfer to naïve recipients indicating development of innate anti-tumor immune memory. In bone marrow myelocytes, GM-CSF, a mediator of cancer-driven emergency myelopoiesis, induced PD-1 expression, phosphorylation and interaction with SHP-2, the Src family kinase Lyn, and GM-CSF receptor beta chain $(\beta c)$, indicating that the PD-1:SHP-2 axis targets a key pathway of myelocyte differentiation. In contrast, SHP-2 ablation or antibody blockade of the PD1/PD-L1 pathway enhanced GM-CSF-mediated phosphorylation of the transcription factors HOXA10 and IRF8 that regulate leukocyte differentiation and monocytic/moDC lineage commitment, respectively. Thus, SHP-2 and the PD-1:SHP-2 axis pose a signaling restrain in myeloid differentiation and 
monocyte/DC lineage commitment thereby preventing the generation of innate anti-tumor immune memory and resulting in a myeloid landscape that suppresses anti-tumor immunity. 


\section{Results}

\section{Myeloid-specific SHP-2 targeting suppresses tumor growth and modifies the immunosuppressive properties of MDSC.}

To dissect the role of PD-1:SHP-2 axis in anti-tumor immune responses, we used mice with conditional targeting of the Ptpn11 gene (encoding for SHP-2), thereafter named Shp2 $2^{\mathrm{f} / \mathrm{f}}$, that significantly depletes SHP-2 expression ${ }^{37}$. We crossed Shp2 $2^{\mathrm{f} / \mathrm{f}}$ mice with mice expressing Cre recombinase under the control of the lysozyme (LysM) promoter to induce selective depletion of the Ptpn11 gene in myeloid cells (Shp2 $\left.2^{\mathrm{f} / \mathrm{f}} \mathrm{LysMCre}\right)$ or with mice expressing Cre recombinase under the control of the distal Lck promoter to induce selective depletion of the Ptpn11 gene in mature T cells (Shp2 $2^{\mathrm{f} / \mathrm{f}} \mathrm{LkCre}$ ). For our studies, first, we used the murine B16-F10 melanoma tumor model because it has been informative in dissecting mechanisms of resistance to checkpoint immunotherapy ${ }^{38}$ and also induces cancer-driven emergency myelopoiesis and output of MDSC ${ }^{23}$. Although no difference in tumor growth was observed between Shp $2^{\mathrm{f} / \mathrm{f}}$ LckCre and control Shp $2^{\mathrm{f} / \mathrm{f}}$ mice, Shp $2^{\mathrm{f} / \mathrm{f}}$ LysMCre mice had significantly reduced tumor growth

(Fig. 1A). Because $\mathrm{CD}^{+} \mathrm{T}$ cells are the mediators of anti-tumor responses, we assessed activation of $\mathrm{CD}^{+} \mathrm{T}$ cells in tumor draining lymph nodes (TDLN) that have been previously determined to be the targets of PD-1-mediated inhibition of anti-tumor $\mathrm{T}$ cell function ${ }^{39,40} \mathrm{CD}^{+} \mathrm{T}$ cells in $\mathrm{TDLN}$ of Shp2 $2^{\mathrm{f} / \mathrm{L}}$ LysMCre mice displayed a more activated state compared to their counterparts in $\operatorname{Shp} 2^{\mathrm{f} / \mathrm{f}} \mathrm{LckCre}$ and control Shp2 $2^{\mathrm{f} / \mathrm{f}}$ mice (Fig. 1B, C; gating strategy, Supplementary Fig. S1). In contrast, no difference was observed in the activation of $\mathrm{TDLN} \mathrm{CD} 8^{+} \mathrm{T}$ cells from $\mathrm{Shp} 2^{\mathrm{f} / \mathrm{L}} \mathrm{LckCre}$ mice compared to control Shp $2^{\text {f/f }}$ tumor-bearing mice (Fig. 1B, C).

We examined the functional properties of MDSCs isolated from mice bearing B16-F10 tumors and found that MDSCs isolated from Shp2/f LysMCre mice had significantly diminished immunosuppressive capacity compared to their counterparts isolated from Shp $2^{\mathrm{f} / \mathrm{f}} \mathrm{LckCre}$ and $\operatorname{Shp} 2^{\mathrm{f} / \mathrm{f}}$ mice 
(Fig. 1D). Consistent with this finding, these cells also had lower expression of CD38 (Fig. 1E, F, G), a hallmark of immunosuppressive MDSC ${ }^{41}$. Thus, SHP-2 ablation switches the fate and function of myeloid cells away from immunosuppressive MDSC in mice bearing B16-F10 tumors.

\section{Improved anti-tumor function induced by myeloid-specific SHP-2 deficiency is not further enhanced by PD-1-blocking immunotherapy.}

Previously, we determined that PD-1 is expressed in myeloid progenitors and immature myeloid cells in tumor bearing mice, whereas PD-1 ablation switches the fate of myeloid cells away from immunosuppressive MDSC toward inflammatory monocytes and DC ${ }^{23}$. Based on these previous observations and our present findings, we sought to examine whether a PD-1:SHP-2 axis might be operative in myeloid cells, and whether it might be involved in the suppression of anti-tumor immunity. To investigate this, first, we examined the therapeutic effect of PD-1 blocking immunotherapy in our experimental mice bearing B16-F10 tumors. Compared to $\operatorname{Shp} 2^{\mathrm{f} / \mathrm{f}}$ mice receiving control $\operatorname{IgG} 2 \mathrm{a}, \operatorname{Shp} 2^{\mathrm{f} / \mathrm{f}}$ mice treated with anti-PD-1 antibody displayed a significant reduction of tumor growth (Fig. 2A, C). In contrast, Shp $2^{\mathrm{f} / \mathrm{f}}$ LysMCre mice that had diminished tumor growth compared to $\mathrm{Shp} 2^{\mathrm{f} / \mathrm{f}}$ control tumor bearing mice (Fig. 2C), did not benefit from treatment with anti-PD-1 antibody compared to treatment with control IgG2a (Fig. 2B, C). In addition, expression of CD38 in MDSC was diminished by anti-PD-1 antibody treatment in tumor-bearing $\mathrm{Shp} 2^{\mathrm{f} / \mathrm{f}}$ control mice but not in Shp $2^{\mathrm{f} / \mathrm{L}} \mathrm{Ly}$ SMCre mice, which expressed lower levels of CD38 than Shp2 $2^{\mathrm{f} / \mathrm{f}}$ tumor-bearing mice, in both IgG2a and anti-PD-1 treatment groups (Fig. 2D, E, F, G). Compared to the Shp $2^{\mathrm{f} / \mathrm{f}}$ tumor-bearing group, Shp $2^{\mathrm{f} / \mathrm{f}}$ LysMCre tumor-bearing mice exhibited enhanced activation and increased recruitment of $\mathrm{T}$ effector $\left(\mathrm{T}_{\mathrm{EF}}\right) \mathrm{CD} 4^{+}$and $\mathrm{CD} 8^{+} \mathrm{T}$ cells (Fig. 2H, I, J, K). Treatment with anti-PD-1 antibody increased the activation of CD8 ${ }^{+}$T cells (Fig. 2H, I) and expansion of T effector cells $\left(\mathrm{T}_{\mathrm{EF}}\right) \mathrm{CD} 4^{+}$and $\mathrm{CD} 8^{+} \mathrm{T}$ cells in Shp2 $2^{\mathrm{f} / \mathrm{f}}$ but not in Shp2 ${ }^{\mathrm{f} / \mathrm{f}} \mathrm{LysMCre}$ tumorbearing mice, which had higher $\mathrm{T}_{\mathrm{EF}}$ cells in TDLN in both IgG2a and anti-PD-1 treatment groups (Fig. 
2J, K). These results suggest that SHP-2 in myeloid cells has an important role in mediating effects of PD1 blockade and, for this reason, myeloid-specific SHP-2 ablation improves anti-tumor immunity which is not further improved by PD-1 blocking immunotherapy. Moreover, SHP-2 expression in myeloid cells has dominant role over SHP-2 expression in T cells in regulating systemic anti-tumor immunity.

\section{Myeloid-specific SHP-2 deficiency alters the differentiation fate of tumor infiltrating myeloid cells, and induces $\mathbf{T}$ cell recruitment and activation.}

To study the effects of SHP-2 ablation in myeloid cells in more detail, we employed the MC17-51 fibrosarcoma mouse tumor model that is well-established to induce robust cancer-mediated emergency myelopoiesis, leading to significant output of bone-marrow-derived MDSCs and TAMs ${ }^{42}$. Consistent with our findings in mice bearing B16-F10 tumors (Fig. 1A and Fig. 2C), we observed significantly diminished tumor growth in Shp2 $2^{\mathrm{f} / \mathrm{f}} \mathrm{LysMcCe}$ mice bearing MC17-51 tumors, compared to control Shp $2^{\mathrm{f} / \mathrm{f}}$ mice (Fig. 3A, B, C, D, Supplementary Fig. S2), but not in Shp2 $2^{\mathrm{f} / \mathrm{L}}$ LckCre mice (Supplementary Fig. S3).

In the mouse, MDSCs consist of two major subsets, CD11b+Ly6C ${ }^{\text {hi }}$ Ly6G $^{-}$monocytic (M-MDSC) and $\mathrm{CD} 11 \mathrm{~b}^{+} \mathrm{Ly}_{6 \mathrm{C}^{\mathrm{lo}}} \mathrm{Ly}_{6 \mathrm{G}^{+}}$polymorphonuclear (PMN-MDSC) ${ }^{8}$. These cells have similar morphology and phenotype to normal monocytes and neutrophils but distinct genomic and biochemical profiles ${ }^{43}$. In humans, in addition to M-MDSC and PMN-MDSC, a small subset of poorly characterized early stage MDSC (eMDSC) has been identified ${ }^{8}$. We did not observe quantitative differences in tumor-infiltrating myeloid cells (Fig. 3E), but Shp2 ${ }^{\mathrm{f} / \mathrm{f}}$ LysMCre mice had an increased fraction of M-MDSC in tumors (Fig. 3F, G) and an increased ratio of M-MDSC: PMN-MDSC (Fig. 3H; gating strategy, Supplementary Figure S3). A similar increase of M-MDSC tumor-infiltrating myeloid cells in B16-F10 tumor-bearing Shp 2 f/f LysMcre mice, which also developed smaller tumors (Supplementary Fig. S4A-E). In the TDLN of MC17-51 tumor-bearing Shp2 $2^{\mathrm{f} / \mathrm{L}} \mathrm{Ly}$ sMCre mice, there was a significant increase of CD4 ${ }^{+}$and $\mathrm{CD} 8^{+} \mathrm{T}_{\mathrm{EF}}$ 
cells (Fig. 3I) and higher activation of $\mathrm{CD}^{+}$T cells (Fig. 3J, K). Additionally, we also observed a systemic increase of $\mathrm{T}_{\mathrm{EF}} \mathrm{CD}^{+}$cells, increase of $\mathrm{T}_{\mathrm{EF}}$ and $\mathrm{T}$ central memory-like $\left(\mathrm{T}_{\mathrm{CM}}\right) \mathrm{CD} 8^{+} \mathrm{T}$ cells (Fig. $\left.3 \mathrm{~L}\right)$ and enhanced activation of $\mathrm{CD}^{+}$and $\mathrm{CD}^{+} \mathrm{T}$ cells (Fig. 3M, N). No differences were noted in the expression of checkpoint receptors including PD-1, PD-L1, CTLA-4, TIGIT or the inducible costimulator (ICOS) in $\mathrm{CD}^{+}$and $\mathrm{CD}^{+} \mathrm{T}$ cells (Supplementary Fig. S5) or in the numbers and activation of Treg in TDLNs (Supplementary Fig. S6). Thus, myeloid-specific Shp2 ablation leads to an increased tumor infiltration by Ly6 $\mathrm{C}^{\text {hi }}$ monocytes and concomitant recruitment and activation of $\mathrm{T}_{\mathrm{EF}}$ and $\mathrm{T}_{\mathrm{CM}}$ cells.

\section{SHP-2 deficiency converts MDSC to differentiated and activated leukocytes.}

Next, we sought to investigate further the properties of MDSC isolated from Shp $2^{\mathrm{f} / \mathrm{L}}$ LysMcre mice. Using mice bearing MC17-51 tumors, we observed that PMN-MDSC from MC17-51 tumor-bearing Shp2 ${ }^{\mathrm{f} / \mathrm{f}}$ LysMCre mice had diminished immunosuppressive function (Fig. 4A) and lower expression of CD38 (Fig. 4B, C). M-MDSC from tumor-bearing Shp2 ${ }^{\mathrm{f} / \mathrm{f}}$ LysMCre also had significantly lower immunosuppressive capacity compared to M-MDSC isolated from Shp2 $2^{\mathrm{f} / \mathrm{f}}$ control tumor-bearing mice (Supplementary Fig. S7). PMN- and M-MDSC subsets are counterparts of granulocytes and monocytes, respectively ${ }^{3}$. Analysis of each of these subsets of MDSC showed that CD11b+Ly6C ${ }^{\text {hi }}$ Ly6G $^{-}$monocytic cells in tumors of Shp2 ${ }^{\mathrm{f} / \mathrm{f}}$ LysMCre mice expressed higher levels of MHC II, CD86 and Ifn- $\gamma$ than their counterparts isolated from Shp2 $2^{\mathrm{f} / \mathrm{f}}$ mice (Fig. 4D, E, F, G), consistent with an activated and proinflammatory phenotype with features of improved antigen presentation and costimulation capacity.

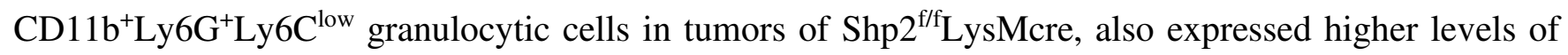
Ifn- $\gamma$ than their counterparts isolated from Shp2 $2^{\mathrm{f} / \mathrm{f}}$ mice (Fig. $\left.4 \mathrm{~F}, \mathrm{G}\right)$. These findings suggest that in the context of cancer, SHP-2 ablation switches the fate of myeloid cells away from immunosuppressive MDSC and promotes the generation of proinflammatory neutrophils, and proinflammatory monocytes with features of enhanced antigen-presentation and $\mathrm{T}$ cell costimulation capacity. 
M-MDSC enter the tumor and convert into TAMs that promote tumor progression ${ }^{3}$. To determine if key myelosuppressive myeloid compartments in tumor-bearing $\operatorname{Shp} 2^{\mathrm{f} / \mathrm{f}}$ LysMcre mice have distinct molecular features, we examined PMN-MDSC and TAMs. We isolated splenic PMN-MDSC from Shp2 $2^{\mathrm{f} / \mathrm{f}}$ and Shp2 $2^{\mathrm{f} / \mathrm{L}}$ LysMCre mice bearing MC17-51 tumors and analyzed gene expression profile by RNA sequencing. Differential gene expression analysis showed that among 17,746 expressed genes, a total of 1,240 genes (842 upregulated; 286 downregulated) were differentially expressed between SHP-2 deficient and control MDSC (Fig. 4H; Supplementary Table 1) indicating that SHP-2 targeting resulted in transcriptionally distinct MDSC populations in tumor bearing mice. Differentially expressed genes from each group are graphically displayed as a heatmap (Fig. 4I). Examination of individual genes showed that among the top differentially expressed genes in MDSC from Shp2 $2^{\mathrm{f} / \mathrm{L}}$ LysMCre mice were genes with important roles in leukocyte differentiation and function ${ }^{44}$. These included multilineage hematopoietic progenitor genes such as Klfl, Gata2 and Egrl and the neutrophil transcription factors Cebpe (c/EBPe) and Gfil (Fig. 4I, J, K; Supplementary Table 1). These findings are consistent with the GMP origin of PMN-MDSC ${ }^{3}$ and the knowledge that progenitor transcription factors are expressed in their progeny ${ }^{45}$. Strikingly, a number of granule genes typically expressed by mature neutrophils, in particular the primary granule proteases neutrophil elastase (Elane), proteinase 3 (Prtn3), cathepsin G (Ctsg), and myeloperoxidase $(\mathrm{Mpo})^{44}$, were highly expressed in myeloid cells isolated from tumor-bearing Shp $2^{\mathrm{f} / \mathrm{L}}$ LysMCre mice (Fig. 4I, L, M, N). In addition, genes expressed in mature, fully differentiated granulocytes such as $S 100 a 8$ and Camp ${ }^{44}$, were elevated in SHP-2 deficient myeloid cells (Fig. 4I; Supplementary Table 1).

Over-representation and Gene Set Enrichment Analysis (GSEA) revealed that relative to those isolated from control tumor-bearing hosts, gene expression profiles of myeloid cells from Shp2 $2^{\mathrm{f} / \mathrm{L}} \mathrm{Ly}$ sMCre tumor-bearing mice were enriched for processes involved in positive regulation of cytokine production, PRC2-EZH2 targets, phagosome formation, myeloid cell differentiation, and neutrophil-mediated immunity, as well as Notch signaling and HOXA targets, and were dominated by functions of antigen 
presentation, myeloid cell migration, mitosis and autophagy (Fig. 4O, P, Q, and Supplementary Fig. S8). Consistent with these, transcripts of the inflammatory mediators S100a8/9, IFN I-inducible genes Rsad2 and Ifit2 ${ }^{46,47}$ as well as Cmpk2 that is required for NLRP3 inflammasome activation downstream of IFNR1 signaling ${ }^{48}$, were significantly elevated in SHP-2 deficient cells (Fig. 4I; Supplementary Table 1). Conversely, expression of Trem2, a myeloid receptor that transmits intracellular signals promoting immunosuppression function in myeloid cells ${ }^{7}$ was significantly decreased (Fig. 4I, R; Supplementary Table 1). SHP-2 deficient MDSC also exhibited lower expression of unfavorable regulators of myeloid function such as $M s r l$ that promotes neutrophil netosis ${ }^{49}$, a protumorigenic process ${ }^{50}$, and $X b p 1$, a classical marker of the unfolded protein response (UPR) that polarizes tumor infiltrating myeloid cells to highly immunosuppressive MDSC ${ }^{51,52}$ (Fig. 4I, S, T; Supplementary Table 1). Together, these results indicate that myeloid cells in tumor-bearing $\mathrm{Shp} 2^{\mathrm{f} / \mathrm{L}} \mathrm{LysMCre}$ mice are skewed away from immunosuppressive MDSC and display features of differentiated neutrophils together with proinflammatory properties of neutrophil-mediated immunity.

\section{SHP-2 deficiency reshapes the intratumoral macrophage infiltrate.}

Next, we examined the effect of SHP-2 targeting on the transcriptional properties of TAMs that are generated from M-MDSC, which convert to macrophages in tumor and have a decisive role in shaping anti-tumor immunity. Differential gene expression analysis showed that among 16,749 expressed genes, a total of 7,307 genes (3,650 upregulated; 3,657 downregulated) were differentially expressed between SHP-2 deficient and control TAMs (Fig. 5A; Supplementary Table 2) indicating that SHP-2 ablation

resulted in transcriptionally distinct TAM populations in tumor bearing mice Shp $2^{\mathrm{f} / \mathrm{f}} \mathrm{LysMCre}$ and $\operatorname{Shp} 2^{\mathrm{f} / \mathrm{f}}$ mice. Differentially expressed genes from each group were graphically displayed in a heatmap (Fig. 5B). TAMs from Shp2 $2^{\mathrm{f} / \mathrm{f}}$ LysMCre mice displayed enhanced expression of genes with important roles in monocyte, macrophage, and DC differentiation and function. These included monocyte specification 
transcription factors $I R F 8$, Klf4 and Zeb2 (Fig. 5B, C, D, E, F, G; Supplementary Table 2) and monocyte signature genes such as $C f p, L y 86$ and $C s f 1 R$ (Supplementary Table 2). There was also increase in gene transcripts of DC specification such as the transcription factor Baft3 (Fig. 5B, H; Supplementary Table 2), which together with $\operatorname{Irf} 8$ and $K l f 4$ is essential for DC differentiation ${ }^{33,53-55}$. TAMs from Shp2 $2^{\mathrm{fl} / \mathrm{fl}}$ LysMCre mice also had increased expression of the immunostimulatory molecules CD86 and CD83 (Fig. 5B, I; Supplementary Table 2), which are associated with moDC differentiation ${ }^{45,56}$. These findings suggest that TAMs in Shp2 $2^{\mathrm{fl} / \mathrm{fl}}$ LysMCre mice have enhanced gene expression programs identifying monocytes differentiated from MDPs that can give rise to Ly6C $\mathrm{C}^{\text {hi }}$ classical and moDC-producing monocytes ${ }^{45}$. This is also supported by the finding that signature transcripts of moDCs such as Csfl, Siglecl, Clec10 and Slamf $7^{57}$ were increased in SHP-2 deficient TAMs (Fig. 5B; Supplementary Table 2). There was elevated expression of Nos2 (Fig. 5B), which characterizes inflammatory macrophages differentiated from Ly6C ${ }^{\text {hi }}$ monocytes ${ }^{58}$. There was also a significant elevation of STING (encoded by Tmem173) (Supplementary Table 2), a pattern recognition receptor that transmits signals activating IFN type I responses ${ }^{59}$, and IFN type I-induced proinflammatory genes Ifit2, Ifit3 and Cmpk2 (Fig. 5B) as well as TLRs and TLR downstream signaling mediators such as $U n c 93 b 1$ and $W d f y l^{60}$ (Fig. 5B, J, K, S; Supplementary Table 2), indicating an enhanced proinflammatory program. Conversely, there was diminished expression of inhibitory genes such as Havcr2 (encoding for Tim3), Prdm1, Trem2 and Wnt (Fig. 5B, L, M, N) all of which have detrimental roles in macrophage and DC function ${ }^{7,61-63}$.

Pathway enrichment analysis, showed that SHP-2 deficient TAMs were enriched for genes of macrophage differentiation and activation, phagocytosis, TLR and NF-kB signaling, cytokine and chemokine activity, IL-1 response, cell killing, antigen presenting function and autophagy (Fig. 5O, P, Q, R, S, Supplementary Fig. S9), all of which are associated with anti-tumor properties of TAMs ${ }^{64-68}$. The differentially expressed cytokines and cytokine production pathways involved the key proinflammatory cytokines IL-1 $\alpha$ IL-1 $\beta$ and IL-18, as well as IL-6, IL-17, IL-10, type I IFN and IFN- $\gamma$ (Fig. 5T, U, V, W, 
Supplementary Fig. S8). In addition, several chemokines were upregulated in SHP-2 deficient TAMs (Supplementary Fig. S8; Supplementary Table 2) including Pf4 (CXCL4) that, besides inducing platelet aggregation, acts as chemoattractant for neutrophils and monocytes, and enhances $\mathrm{T}$ memory cell responses ${ }^{69,70}$; CCL2, CCL3, CCL4, CCL6, CCL7, CCL8, CCL9, CXL2 and CXL3 which collectively promote monocyte, DC, NK and T cell recruitment, and macrophage activation, thereby promoting a proinflammatory immune response ${ }^{71-75}$. TAMs from Shp2 $2^{\mathrm{f} / \mathrm{L}}$ LysMCre mice had an enhanced signature of multiple metabolic pathways including lipid, carbohydrate and amino acid transport, cholesterol metabolism, and energy metabolism (Fig. 5X; Supplementary Table 2). Enhanced metabolic activity, characterized of glucose and glutamine metabolism, increased levels of amino acids, and anabolic lipid metabolism was also observed in phagocytes generated from bone marrow progenitors of Shp $2^{\mathrm{f} / \mathrm{f}} \mathrm{LysMCre}$ mice (Supplementary Fig. S10). Consistent with these findings, GSEA using Gene Ontology Biological Processes Pathways gene sets showed that TAMs from Shp2 $2^{\mathrm{f} / \mathrm{L}}$ LysMCre mice were characterized by highly expressed signatures of leukocyte activation, chemotaxis, migration, cytokine production, inflammatory response, and tissue remodeling (Supplementary Fig. S11).

\section{SHP-2 ablation induces innate immune memory and long-lasting anti-tumor properties in monocytes.}

Our RNA-seq studies showed that SHP-2 deficient neutrophils in tumor-bearing mice have a gene program of effector myeloid cells enriched in gene transcripts involved in neutrophil differentiation and specification (Fig. 4; Supplementary Table 1) indicating that they are originated from GMPs ${ }^{44,45}$. In parallel, our RNA-seq studies of SHP-2 deficient TAMs showed enhanced expression of genes characteristic of monocytes differentiated from MDPs that can give rise to Ly6C ${ }^{\text {hi }}$ classical and moDCproducing monocytes ${ }^{45}$. These findings suggest that altered tumor-driven emergency myelopoiesis in 
Shp $2^{\mathrm{f} / \mathrm{L}}$ LysMCre mice results in increased output from two different progenitor stages, GMPs and MDPs (Fig. 6A).

Metabolism-driven changes, including glucose, glutamine and cholesterol metabolism, in monocytes and macrophages, lead to a state of long-lasting epigenetic imprinting of functional immune memory named innate immune memory or trained immunity ${ }^{76}$. Other studies have reported that neutrophils may also develop trained immunity, characterized by epigenetic rewiring of gene activity, and can provide long-lasting protection against infections and cancer ${ }^{77-79}$. Our results showed that TAMs from tumor-bearing Shp $2^{\mathrm{f} / \mathrm{f}}$ LysMCre mice have enhanced metabolic activity characterized by upregulation of pathways related to glucose and lipid transport, and cholesterol metabolism (Fig. 5X) that are involved in training ${ }^{80-82}$. In addition, in tumor-bearing $\operatorname{Shp} 2^{\mathrm{f} / \mathrm{f}}$ LysMCre mice, neutrophils exhibited increased expression of Ezh2 methyltransferase, and EZH2 targets (Fig. 4O; Supplementary Table 1), whereas TAMs, which are generated from bone marrow-derived monocytes, exhibited increased expression of Ezh1, as well as Kdm5 family demethylases (Supplementary Table 2), all of which regulate histone and DNA methylation status ${ }^{83,84}$. These findings are indicative of enhanced epigenetic activity in both neutrophils and TAMs in tumor-bearing Shp2 ${ }^{\mathrm{f} / \mathrm{L}}$ LysMCre mice. For these reasons, we examined whether neutrophils or monocytes could mediate long-lasting anti-tumor protection indicating development of trained immunity.

We implanted MC17-51 tumors in Shp2 ${ }^{\mathrm{fl} / \mathrm{fl}}$ LysMCre mice and, nine days later, collected

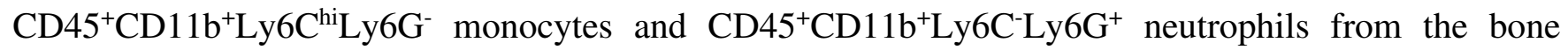
marrow of tumor-bearing mice and adoptively transferred them into naïve WT tumor bearing hosts implanted with MC17-51 tumors (Fig 6B), as previously described ${ }^{79}$. Compared to control tumor-bearing mice, no difference in tumor growth was observed in mice that received neutrophils from $\operatorname{Shp} 2^{\mathrm{f} / \mathrm{L}} \mathrm{LysMCre}$ tumor bearers whereas the monocyte recipient group had significantly reduced tumor growth (Fig. 6C, D). These results show that the enhanced anti-tumor immunity in Shp2 $2^{\mathrm{f} / \mathrm{f}}$ LysMCre is mediated by Ly6C ${ }^{\text {hi }}$ 
monocytes generated in the bone marrow during tumor-driven emergency myelopoiesis. Moreover, the anti-tumor properties of SHP-2 deficient Ly6C ${ }^{\text {hi }}$ monocytes are long-lasting and can be transferred to naïve tumor-bearing hosts.

\section{SHP-2 and canonical PD-1:SHP-2 signaling impede phosphorylation of HOXA10 and IRF8 transcription factors that promote myeloid cell differentiation and monocyte lineage commitment.}

Next, we sought to determine the mechanistic basis of the immunological outcomes induced by myeloid-specific SHP-2 targeting. Activating SHP-2 mutations prevent myeloid cell differentiation and lead to the accumulation of immature myelocytes and development of leukemia ${ }^{25}$. Established SHP-2 targets in myeloid progenitors include the transcription factors HOXA and IRF8. During growth factorinduced myelopoiesis, as differentiation proceeds, tyrosine phosphorylation of HOXA10 decreases its binding affinity for target gene promoters that are repressed by HOXA10 binding. SHP-2-mediated dephosphorylation of HOXA prevents derepressing of HOXA target genes and myeloid cell differentiation 27,31. SHP-2 also regulates the function of IRF8 because SHP-2-mediated dephosphorylation of IRF8 prevents its nuclear localization and transcriptional activation, which is required for monocyte lineage differentiation ${ }^{35,36}$. Our RNA-seq studies a showed that myeloid cells isolated from tumor-bearing $\operatorname{Shp} 2^{\mathrm{fl} / \mathrm{fl}}$ LysMCre mice had enhanced neutrophil differentiation and increased HOXA targets (Fig. 4), whereas TAMs exhibited highly elevated expression of transcripts relevant to monocyte and mo/DC differentiation including Irf8, Kl4, Zeb2 and Batf3 (Fig. 5B). Based on these findings, we examined whether phosphorylation of HOXA10 and IRF8 might be altered in SHP-2 deficient bone marrow myeloid cells cultured with GM-CSF+IL-3 27,31. Flow cytometric analysis confirmed that by $72 \mathrm{hr}$ of culture $\geq 95 \%$ of the Lin $^{\text {pos }}$ cells were myelocytes (Supplementary Fig. S12A). HOXA10 immunoprecipitation followed by immunoblot with anti-phosphotyrosine antibody showed enhanced HOXA10 phosphorylation in SHP-2 deficient myelocytes (Fig. 7A). Similarly, IRF8 
immunoprecipitation followed by anti-phosphotyrosine immunoblot showed that SHP-2 deficient cells had increased IRF8 phosphorylation (Fig. 7B). These results provide a mechanistic basis explaining how SHP-2 ablation promotes the generation of differentiated myeloid cells that is mediated by enhanced HOXA10 phosphorylation, and enhances monocytic lineage output that is regulated by phosphorylation of IRF8, which drives the transcriptional program of monocyte and DC lineage differentiation. Because as we observed previously, after culture with GM-CSF myeloid progenitors and their progeny express PD1 23, we examined whether PD-1:SHP-2 interaction might be detected in myelocytes. PD-1 immunoprecipitation followed by SHP-2 immunoblot detected a robust PD-1:SHP-2 interaction in control cells that was abrogated in SHP-2 deficient myelocytes (Fig. 7C).

Our previous studies showed that GM-CSF-mediated activation of Erk and mTOR is enhanced in PD-1 deficient CMPs and GMPs during in vitro culture ${ }^{23}$. Because Erk and mTOR are well-established targets of PD-1 in T cells ${ }^{85}$, we examined whether a canonical PD-1:SHP-2 signaling might impact GMCSF signaling in myelocytes. We used primary bone marrow cells and cultured them with GM-CSF and IL-3. During culture, there was a decrease of Lin $^{\text {neg }}$ and increase of Lin ${ }^{\text {pos }}$ cells (Fig. 7D), as expected. In both Lin $^{\text {neg }}$ and Lin ${ }^{\text {pos }}$ cells, PD-1 (Fig. 7E, F, I, J) and PD-L1 (Fig. 7G, H, K, L; Supplementary Fig. S12B, C) were expressed at low levels before treatment and were upregulated after culture. In T cells, SHP-2 is recruited to PD-1 cytoplasmic tail after tyrosine phosphorylation by TCR-mediated activation of Src family kinases Fyn and Lck ${ }^{20,86}$. In the myeloid lineage, the $\beta c$ subunit of the GM-CSF receptor (GM-CSFR), shared also by the IL-3 and IL-5 receptors, is the major signaling subunit and is tyrosine phosphorylated in response to cytokine stimulation ${ }^{87}$. The Src family member, Lyn, can directly associate with GM-CSFR $\beta$ c subunit ${ }^{88}$. We examined if PD-1 is phosphorylated by GM-CSF at tyrosine Y248 within the conserved ITSM motif of PD-1 cytoplasmic tail, a site known to be phosphorylated by Src family kinases leading to PD-1 interaction with SHP-2 in T cells ${ }^{20,86}$. Immunoprecipitation with anti-PD1 antibody followed by immunoblot with phospho-specific PD-1 antibody that recognizes pY248 ${ }^{20,89}$, 
showed that GM-CSF induced PD-1 phosphorylation and interaction with SHP-2 (Fig. 7M). Sequential immunoblot with Lyn-specific antibody and GM-CSFR $\beta \mathrm{c}$-specific antibody showed that both proteins were detected in PD-1 immunoprecipitations (Fig. 7M). Thus, a canonical PD-1:SHP-2 axis is activated in myeloid cells during GM-CSF-mediated signaling, resulting in the recruitment of PD-1 inhibitory machinery to a major signaling receptor involved in myeloid cell activation, proliferation and differentiation.

Because our results showed PD-1 and PD-L1 upregulation and PD-1:SHP-2 interaction in myeloid cells, we examined whether activation of PD-1:SHP-2 axis by PD-1 and PD-L1 receptor-ligand interaction during culture with GM-CSF might pose a signaling restrain to GM-CSF-mediated phosphorylation of HOXA10 and/or IRF8 and whether this might be prevented by PD-1:PD-L1 blockade, similarly to SHP2 depletion. Addition of a PD-L1 blocking antibody during culture enhanced HOXA10 phosphorylation (Fig. 7N) but more potently enhanced IRF8 phosphorylation (Fig. 7O). Thus, SHP-2 and, to a lesser extent, the PD-1:SHP-2 axis pose a signaling restrain in myeloid cell differentiation and lineage fate commitment to monocytes and DCs that depends on IRF8 expression and phosphorylation ${ }^{33,55}$. 


\section{Discussion}

Our present studies reveal a previously unidentified mechanistic role of SHP-2 and the PD-1:SHP2 axis in regulating myeloid cell differentiation, and lineage fate commitment and function in the context of cancer. We found that SHP-2 poses a restrain in GM-CSF-mediated phosphorylation of HOXA10 and IRF8 transcription factors that induce myeloid differentiation and monocyte/DC lineage commitment, respectively ${ }^{90,91}$. In the myeloid lineage, the $\beta c$ subunit of the GM-CSFR, shared also by the IL-3 and IL5 receptors, is the major signaling subunit that is tyrosine phosphorylated by cytokine stimulation and mediates myelocyte differentiation, expansion and activation in response to growth factors ${ }^{87}$. Although JAK2 is the primary kinase activated downstream of GM-CSFR, the Src family member Lyn, can directly interact with GM-CSFR $\beta$ c subunit ${ }^{88}$. We found that PD-1 is phosphorylated by GM-CSF at tyrosine Y248 within the conserved ITSM motif of its cytoplasmic tail, a site known to be phosphorylated by Src family kinases leading to PD-1 interaction with SHP-2 in T cells ${ }^{20,86}$. Moreover, co-engagement of the PD-1:PD-L1 pathway during growth factor engagement of GM-CSFR restrains GM-CSF-mediated phosphorylation of HOXA10 and IRF8, which is reversed by a PD-L1 blocking antibody. These results explain why PD-1 ${ }^{23}$ or SHP-2 ablation in myeloid cells, as shown in the present study, lead to enhanced monocyte and moDC differentiation.

Using cell-specific targeting of SHP-2 in T cells or myeloid cells, we determined that myeloidspecific PD-1:SHP-2 signaling has a dominant role over $\mathrm{T}$ cell-specific PD-1:SHP-2 signaling in regulating anti-tumor immunity in vivo explaining why mice with T cell-specific SHP-2 deficiency do not have improved anti-tumor protection, and retain unaltered therapeutic responses to anti-PD-1 treatment 21. Our data reveal that a canonical PD-1:SHP-2 signaling axis is operative in myeloid cells, similarly to what has been previously established for B and T lymphocytes ${ }^{18,20,92}$. This myeloid-specific PD-1:SHP-2 axis becomes particularly important in the context of cancer, where growth factors released by cancer cells, such as GM-CSF and IL-3 that induce emergency myelopoiesis, directly upregulate PD-1 and PD- 
L1 expression in myeloid progenitors and their progeny, as determined in our present studies, thereby posing a signaling restrain to their effector differentiation and function. This might be the earliest critical signaling target of the PD-1:PD-L1 pathway in the context of cancer, because soluble factors produced by cancer cells can act systemically at early stages of the cancer immunity cycle ${ }^{1}$, before local tumor growth and infiltration by immune cells that are subject to inhibitory signals in the immunosuppressive tumor microenvironment.

We found that targeting SHP-2 in myeloid cells resulted in enhanced differentiation of neutrophils, and TAMs that are generated from bone marrow-derived monocytes in tumors. Neutrophils were skewed away from immunosuppressive MDSC, and tumor-infiltrating macrophages were skewed away from protumorigenic TAMs that promote tumor growth. Instead, both cell types were characterized by gene signatures and activation of pathways linked to effector differentiation and activation, and by biological properties of enhanced proinflammatory function, cytokine production, phagocytosis, antigen presentation, and autophagy, an integral component of the antigen presentation process ${ }^{93}$. This differentiation program of myeloid cells was associated with potent anti-tumor function, diminished tumor growth, and enhanced recruitment, activation and effector differentiation of $\mathrm{T}$ cells, despite unaltered expression of SHP-2 and multiple checkpoint inhibitors in T cells. Several chemokines, such as CCL3, CCL4, CCL6, CCL7, CCL8, CCL9, CXCL2 and CXCL3 that displayed increased expression in SHP-2 deficient TAMs, can induce chemotaxis, transendothelial migration, extravasation and recruitment of monocytes, macrophages, and neutrophils to tumors ${ }^{71-74,94}$. Although previous studies have reported that increased production of such chemokines by TAMs might have a tumor-promoting effect by recruiting immunosuppressive myeloid cells to tumors ${ }^{95,96}$, increase of these chemokines in Shp2 ${ }^{\mathrm{f} / \mathrm{L}} \mathrm{LysMCre}$ tumorbearing mice was associated with diminished tumor growth. This outcome can be explained by the differentiated and activated features of myelocytes in Shp $2^{\mathrm{f} / \mathrm{L}} \mathrm{LysMCre}$ tumor-bearing mice that are skewed away from immunosuppressive MDSCs and TAMs and, instead, have enhanced antigen presenting 
functions, phagocytotic and cell killing properties thereby further promoting anti-tumor immune responses instead of tumor growth.

$\mathrm{T}$ cells are responsive to chemokines whose production is controlled by type I IFN signaling produced by innate immune cells ${ }^{97}$. In most cases, this is initiated by viral infection of a cell and detection of pathogenic nucleic acids by intracellular DNA- and RNA-sensing systems such as cGAS/STING, AIM2, and TLR $3{ }^{98}$. IFN type I is also produced by DCs following stimulation of TLR7 and TLR9, which detect viral RNA and DNA molecules, respectively, that have been endocytosed or sequestered by autophagy. The production of type I IFNs in DC can be further amplified by a positive feedback loop that involves the transactivation of IRF7 and IRF8 in response to IFN $\alpha / \beta$ receptor (IFNAR) signaling ${ }^{99}$. Although it was initially thought that type I IFNs exert direct anticancer effects by activating IFNAR signaling in malignant cells hence inhibiting cell cycle progression ${ }^{100}$, promoting terminal differentiation 101 or inducing apoptosis ${ }^{102}$, it is becoming increasingly clear that type I IFNs mainly function by stimulating anticancer immune responses via autocrine or paracrine signaling circuits by IFN-stimulated genes ${ }^{103,104}$. These have an instrumental role in the production of IFN-dependent chemokines that lead to preferential recruitment and retention of systemic $\mathrm{CD}^{+} \mathrm{T}$ cells in the tumor, an outcome that can be recapitulated by treatment with type I IFN or DNA damaging chemo- and radiotherapies ${ }^{105}$. In our work, we found that SHP-2 deficient TAMs displayed a significant increase of STING (Supplementary Table 2), TLR signaling (Fig. 5S), and type I IFN (Fig. 5T), and these were paralleled by increase of chemokines that attract and retain activated T cells, such as CCL2, CCL7 and CCL8, leading to systemic recruitment of $\mathrm{CD}^{+} \mathrm{T}$ cells in tumor bearing mice (Supplementary Fig. S9; Supplementary Table 2; Fig. 3I, L).

Previous studies used allosteric inhibitors of SHP-2 such as SHP099, TNO155 106-108 and RMC4550 ${ }^{109}$ with the purpose to target cancer cells, in which SHP-2 is activated downstream of RTK/Ras signaling and functions as an oncogene promoting cancer cell growth ${ }^{106}$, or to target both cancer and immune cells ${ }^{108,109}$. These studies observed upregulation of three CXCR3 ligands, specifically CXCL9, 
CXCL10, CXCL11 in tumor cells thereby leading to $\mathrm{T}$ cell recruitment ${ }^{108}$. Other investigators reported that SHP-2 ablation in macrophages potentiated production of CXCL9 in response to IFN- $\gamma$, thereby facilitating tumor infiltration by $\mathrm{T}$ cells ${ }^{110}$. Combined approaches of SHP-2 allosteric inhibitors together with pharmacologic RTK inhibitors or $\mathrm{KRAS}^{\mathrm{G} 12 \mathrm{C}}-\mathrm{GDP}$ inhibitors have been employed with the main purpose of targeting signaling vulnerabilities of cancer ${ }^{108,111,112}$. These studies have reported that such treatments, by changing the properties of cancer, also alter immune cells of the TME, including increase of $\mathrm{CD}^{+} \mathrm{T}$ cells, depletion of pro-tumorigenic M2 TAMs via attenuation of Csf1 signaling ${ }^{109}$, increase of M1 TAMs and reduced immunosuppression function of MDSCs ${ }^{107,108,112}$, but the mechanisms remained elusive. Our present studies employing a genetic approach to dissect the effects of SHP-2 targeting at multiple levels, showed that myeloid-intrinsic but not T cell-intrinsic depletion of SHP-2 has a dominant anti-tumor effect by altering the myeloid lineage fate, thereby promoting the generation of differentiated granulocytes, and monocytes that became TAMs with signatures of activated antigen presenting cells. In SHP-2 deficient TAMs, we observed enhanced expression of Csf1, together with Slamf7, Siglec1, and Clec10 that form a signature of moDCs ${ }^{57}$. Notably, our results showed that classical M1 but also M2 genes, such as PPAR $\gamma$ and CD206, were increased in SHP-2 deficient TAMs (Supplementary Table 2). Because M2 macrophages are required for resolution of inflammation and tissue remodeling ${ }^{12,113-115}$, the combined actions of M1 and M2 macrophages induced by SHP-2 depletion might be involved in the enhanced anti-tumor function by promoting proinflammatory but also tissue remodeling properties of tumor infiltrating macrophages that induce a pro-resolving-mediated suppression of tumor growth ${ }^{115}$.

An important finding of our studies was the SHP-2 targeting in myeloid cells resulted in longlasting anti-tumor properties of monocytes isolated from the bone marrow of $\operatorname{Shp} 2^{\mathrm{f} / \mathrm{L}} \mathrm{LysMCre}$ tumorbearing mice that could be transferred to naïve tumor-bearing hosts, consistent with the development of trained immunity. Until recently, it was assumed that the innate immune system cannot build immunological memory. The observations that innate immune cells can exert adaptive characteristics and 
can build a non-specific memory, challenged this paradigm ${ }^{116-118}$. Cells of the innate immune system, such as monocytes, can build memory during their differentiation to macrophages, a process named trained (innate) immunity in which signaling, metabolic and epigenetic changes represent the molecular substrate $^{119}$. Neutrophils may also develop trained immunity and provide long-lasting protection against cancer ${ }^{79}$. Emergency myelopoiesis is an integral part of trained immunity because this process develops during the differentiation of myeloid progenitors in the bone marrow ${ }^{120,121}$. Our results showed that SHP2 deficiency resulted in enhanced signaling of bone marrow myeloid progenitors in response to factors of emergency myelopoiesis (Fig. 7), altered metabolic programs of TAMs and bone marrow derived phagocytes (Fig. 5X; Supplementary Fig. S10), and led to increased expression of Ezh1 and Kdm5 epigenetic regulators in TAMs, findings consistent with signaling, metabolic and epigenetic processes associated with trained immunity ${ }^{76,119,122}$. By transferring individually neutrophils and monocytes from the bone marrow of $\mathrm{Shp} 2^{\mathrm{f} / \mathrm{f}}$ LysMCre tumor-bearing mice subjected to cancer-mediated emergency myelopoiesis in vivo, we found that monocytes but not neutrophils displayed properties of long-lasting innate immune memory against tumor transferrable to naïve tumor-bearing hosts. Of note, IL- $1 \beta$ that drives long-term training of monocyte precursors ${ }^{121}$, was highly increased in SHP-2 deficient TAMs, providing evidence for an additional mechanism responsible for the development of trained immunity in monocytes. Together with our findings that SHP-2 and the PD-1:SHP-2 axis restrain the phosphorylation of HOXA10 that regulates myelocyte differentiation, but also the phosphorylation of IRF8 that guides monocyte/DC lineage fate commitment, these data indicate that SHP-2 and PD-1:SHP-2 axis might preferentially have a long-lasting functional impact in the monocytic compartment. It is tempting to speculate that the long-lasting effects of PD-1-based immunotherapy in some, but not all, patients might be related to the development of immunotherapy-mediated monocyte differentiation and trained immunity vs. generation of immunosuppressive MDSCs and TAMs during cancer-driven emergency myelopoiesis. Further studies will investigate these new directions of central regulation of anti-tumor responses to cancer immunotherapy in patients. 
In conclusion, our results provide multiple levels of evidence that SHP-2 and the PD-1:SHP-2 axis pose a signaling restrain to the differentiation and monocyte/DC lineage commitment of myeloid progenitors by suppressing the phosphorylation of the transcription factors HOXA10 and IRF8, resulting in a myeloid landscape that compromises anti-tumor immunity. SHP-2 depletion promotes myelocyte differentiation, activation and antigen presenting function leading to T cell recruitment and activation, and potent anti-tumor immunity. 


\section{Material and Methods}

\section{Animals}

All animal procedures were approved by the Institutional Animal Care and Use Committee (IACUC) at Beth Israel Deaconess Medical Center (Boston MA), and were in accordance with National Institutes of Health Guidelines for the Care and Use of Animals. Mice with conditional targeting of the Ptpn11 gene (encoding for Shp-2), thereafter named Shp2 $2^{\mathrm{f} / \mathrm{f}}$, were kindly provided by Dr. Gen-Sheng Feng (University of California, San Diego) and have been previously described ${ }^{37}$. We crossed Shp $2^{\mathrm{f} / \mathrm{f}}$ mice with mice expressing Cre recombinase under the control of the lysozyme (LysM) promoter to induce selective ablation of the Ptpn11 gene in myeloid cells (Shp2 $\left.{ }^{\mathrm{f} / \mathrm{f}} \mathrm{LysMCre}\right)$ or with mice expressing Cre recombinase under the control of the distal Lck promoter to induce selective ablation of the Ptpn11 gene in mature T cells (Shp2 $\left.2^{\mathrm{f} / \mathrm{f}} \mathrm{LckCre}\right)$. C57BL/6 mice and OTI-TCR transgenic mice (C57BL/6-Tg(TcraTcrb)1100Mjb/J) were purchased from Jackson Laboratory (Bar Harbor, Maine).

\section{Tumor cell lines and tumor experiments}

B16-F10 melanoma and MC17-51 fibrosarcoma cell lines were purchased from ATCC. B16-F10 cell line was subcloned and subclones with intermediate growth rate were selected for use. Eight to 12 weeks old mice were used for tumor implantation, and B16-F10 melanoma $\left(1 \times 10^{5}\right.$ or $3 \times 10^{5}$ cells per mouse $)$ or MC17-51 fibrosacroma ( $1 \times 10^{5}$ cells per mouse) were injected subcutaneously in the left flank under isoflurane anesthesia. For adoptive transfer of monocytes or neutrophils into naïve hosts,

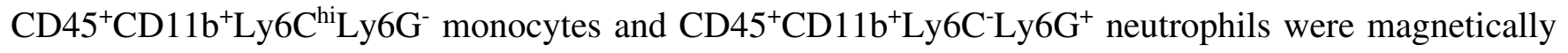
collected from the bone marrow tumor bearing Shp2 ${ }^{\mathrm{f} / \mathrm{f}} \mathrm{LysMCre}$; $0.75 \times 10^{5} \mathrm{MC} 17-51$ tumor cells were mixed with $0.75 \times 10^{5}$ monocytes or $0.75 \times 10^{5}$ neutrophils and were injected subcutaneously in wild type C57BL/6 mice. Starting from day 9, tumor size was monitored every 2 days by a caliper fitted with a Vernier scale. The tumor volume was calculated by the formula tumor volume $=0.5 * \mathrm{width}^{2} *$ length. Mice were euthanized on day 14-16 after tumor implantation and bone marrows, spleens, tumors and tumor- 
draining lymph nodes were harvested. At termination, tumor weight was also measured. For antibody treatment experiments, $250 \mu \mathrm{g}$ of either anti-PD-1 (clone RMPI-14, BioXCELL) or IgG2a control (clone 2A3, BioXCell) diluted in sterile PBS were injected intraperitoneally in a volume of $100 \mu 1$ per mouse on days 9,11 , and 13 after tumor inoculation and the mice were euthanized at day 15 .

\section{Cell purification and processing}

Single cell suspensions were made from spleens, tumor draining lymph nodes, and tumors as previously described ${ }^{23}$. Briefly, tumors were digested by $1 \mathrm{mg} / \mathrm{ml}$ of Collagenase I in incomplete RPMI and then filtered through $70 \mu \mathrm{l}$ strainers, while the spleens and the tumor draining lymph nodes were directly filtered through $70 \mu \mathrm{l}$ strainers. For flow cytometry studies, $1 \times 10^{6}$ were resuspended in PBS $1 \mathrm{X}$ supplemented

with $2.5 \%$ FBS, and plated in 96-well round bottom plates. Surface staining was performed at $4^{0} \mathrm{C}$ for 25 30 minutes with the flow antibodies listed in Supplementary Table 3. For intracellular staining, Foxp3 / Transcription Factor permeabilization/staining Buffer set (Thermo Fischer) was used according to the manufacturer's instructions. Intracellular staining was performed at $4^{0} \mathrm{C}$ for $35-45$ minutes with flow antibodies listed in Supplementary Table 3. Cells were acquired using Becton Dickinson LSR Fortsessa or Beckman-Coulter Cytoflex Flow cytometer and analyzed with Flowjo Software.

\section{Suppression assay}

MDSC-mediated suppression was assessed using previously established methodology ${ }^{23}$. Briefly, splenic MDSCs were isolated from the spleens of mice bearing B16-F10 melanoma or MC17-51 fibrosarcoma, by using either the EasySep ${ }^{\mathrm{TM}}$ Mouse MDSC $\left(\mathrm{CD} 11 \mathrm{~b}^{+} \mathrm{GR} 1^{+}\right)$Isolation Kit (StemCell Technologies) or with MDSC isolation kit (Miltenyi Biotech) to separate GR1 ${ }^{\text {hi }} \mathrm{Ly}^{+} \mathrm{G}^{+} \mathrm{Ly}_{6} \mathrm{C}^{-}$(PMN-MDSC) and GR1 ${ }^{\operatorname{dim}}{ }^{2 y 6 G}{ }^{-} \mathrm{Ly}^{+}(\mathrm{M}-\mathrm{MDSC})$ cells. Serial dilutions of MDSCs $\left(2 \times 10^{5}, 1 \times 10^{5}, 0.5 \times 10^{5}, 0.25 \times 10^{5}\right.$, $0.125 \times 10^{5}$ ) were plated in flat bottom 96-well plates with $2 \times 10^{5}$ splenocytes per well isolated from OTI- 
TCR transgenic mice and $250 \mathrm{ng} / \mathrm{ml}$ of ovalbumin peptide $\left(\mathrm{OVA}_{257-264}\right)$ for 72 hours. As control, OTI splenocytes were incubated with OVA peptide (OVA257-264) without MDSC. ${ }^{3} \mathrm{H}$-thymidine was added for the last 16 hours of a $72 \mathrm{hr}$ culture, and thymidine incorporation was measured by MicroBeta plate counter (Perkin Elmer).

\section{Cell culture and signaling}

For signaling experiments, bone marrow cells from C57BL/6 WT mice or Shp2 $2^{\mathrm{f} / \mathrm{f}}$ and Shp $2^{\mathrm{f} / \mathrm{f}}$ LysMCre mice were cultured for $48 \mathrm{hr}$ in Iscove's media supplemented with 10\% fetal bovine serum, $2 \mathrm{mmol} / \mathrm{L}$ glutamine, 100 units/mL penicillin-streptomycin, $10 \mathrm{mM}$ Hepes and $20 \mu \mathrm{M}$ beta-mercaptoethanol, in the presence of GM-CSF (10 ng/ml) and IL-3 (5 ng/ml) (both purchased from Peprotech). Where indicated, a PD-L1 blocking antibody (MIH5) $(10 \mu \mathrm{g} / \mathrm{ml})$ was added in the cultures. For studies of GM-CSF-mediated short-term signaling activation, $48 \mathrm{hr}$ after culture as described above, cells were rested for $3 \mathrm{hr}$ at $37^{\circ} \mathrm{C}$ in RPMI-1640 containing $10 \mathrm{mM}$ HEPES. Cells were then either left unstimulated or were resuspended at $10 \times 10^{6}$ cells/ml in pre-warmed RPMI 1640 containing $10 \mathrm{mM}$ HEPES and were stimulated with GMCSF (40 ng/ml). At the indicated time points, the reaction was stopped by adding cold PBS and placement on ice.

\section{Immunoprecipitation and immunoblotting}

To prepare lysates, cells were washed in PBS and lysed as previously described ${ }^{123}$. Briefly, cells were resuspended and lysed in lysis buffer containing $50 \mathrm{mM}$ Tris- $\mathrm{HCl}, \mathrm{pH}$ 7.4, $150 \mathrm{mM} \mathrm{NaCl,} 2 \mathrm{mM} \mathrm{MgCl}_{2}$, $10 \%$ glycerol and 1\% NP-40 supplemented with $2 \mathrm{mM}$ sodium orthovanadate, $1 \mathrm{mM}$ sodium fluoride, 1 mM phenylmethylsulfonyl fluoride (PMSF), and protease Inhibitor Cocktail (Thermo Scientific). Cell lysates were resolved by SDS-PAGE, transferred on nitrocellulose membrane, and analyzed by Western 
blotting with the indicated antibodies. The following antibodies were used for Western blotting: SHP-2 (D50F2) cat\# 3397T, Cell Signaling Technology; Lyn (H-6) Cat\# sc-7274 AF790, Santa Cruz Biotechnology; HoxA10 (E-11) Cat\# sc-271428 AF680, Santa Cruz Biotechnology; ICSBP (IRF8) (E-9) Cat\# sc-365042 AF680, Santa Cruz Biotechnology; Pdcd-1 (RMP1-30) Cat\# sc-56200 AF680, Santa Cruz Biotechnology; IL-3/IL-5/GM-CSFRß (A-3) Cat\# sc-398246, Santa Cruz Biotechnology; p-Tyr (PY99) Cat\# sc-7020 AF790, Santa Cruz Biotechnology. The rabbit polyclonal anti-phospho-Y248 (ITSM) PD1 (pPD-1) antibody was developed in our laboratory ${ }^{89}$. For conjugation of pPD-1 Ab the Li-COR IRDye 800CW protein labeling kit for high molecular weight and microscale reactions (Cat\# 829-08881) was used. Immunoprecipitations were performed with agarose-conjugated antibodies HoxA10 (E-11) Cat\# sc271428 AC, Santa Cruz Biotechnology; ICSBP (IRF8) (E-9) Cat\# sc-365042 AC, Santa Cruz Biotechnology; Pdcd-1 (RMP1-30) Cat\# sc-56200 AC, Santa Cruz Biotechnology. Briefly, $20 \mu 1$ of agarose slurry/sample were first washed three times with lysis buffer and then were resuspended in $40 \mu 1$ of buffer. For each IP sample, $40 \mu \mathrm{l}$ of washed agarose-conjugated Ab were mixed with 500-1000 $\mu \mathrm{g}$ of cell lysates and incubated overnight at $4^{\circ} \mathrm{C}$ with gentle rotation. The agarose slurry was then washed three times with lysis buffer and boiled 5 min in denaturing sample buffer followed by quick spin. The supernatant was analyzed by SDS-PAGE, transferred to a nitrocellulose membrane and blotted with the indicated antibodies. Images were visualized, acquired and quantified with Li-COR Odyssey CLx imaging system.

\section{RNA sequencing and analysis}

For RNA sequencing, PMN-MDSCs $\left(\mathrm{CD} 11 \mathrm{~b}^{+} \mathrm{GR} 1^{\mathrm{hi}} \mathrm{Ly} 6 \mathrm{G}^{+}\right)$were isolated from the spleens of MC17-51 fibrosarcoma bearing and $\operatorname{Shp} 2^{\mathrm{f} / \mathrm{f}}$ LysMCre and $\mathrm{Shp} 2^{\mathrm{f} / \mathrm{f}}$ mice by magnetic bead isolation. TAMs were isolated from the same mice by cell sorting after using the Live/Dead Fixable Far Read Dead Cell Stain kit (ThermoFisher; L34973) to identify live cells, staining with antibodies specific for CD45, CD11b, F4/80 and Ly6G and gating on $\mathrm{CD}_{4} 5^{+} \mathrm{CD} 11 \mathrm{~b}^{+} \mathrm{F} 4 / 80^{+} \mathrm{Ly} 6 \mathrm{G}^{-}$live cells. Total RNA was isolated from the 
cells using the Qiagen RNeasy Mini Kit (Qiagen, cat\# 74104). For each sample, 400 ng of total RNA was then used in Illumina's TruSeq Stranded mRNA Library kit (Cat\# 20020594) for polyA mRNA isolation and library construction. Libraries were sequenced on Illumina NextSeq 500 as paired-end 42-nt reads (Active Motif). Raw sequencing reads were quality-checked using FastQC (v0.11.5) ${ }^{124}$ and data were pre-processed with Cutadapt (v2.5) ${ }^{125}$ for adapter removal following best practices ${ }^{126}$. Gene expression quantification was performed by aligning against the GRCm38 genome using STAR (v2.7.3a) ${ }^{127}$ and quantifying reads against Ensembl v98 ${ }^{128}$ annotated gene loci with featureCounts (Subread 1.6.2) ${ }^{129}$. Differential gene expression analysis was performed using DESeq2 (v1.24.0) ${ }^{130}$, while ClusterProfiler (v3.12.0) ${ }^{131}$ was utilized for downstream functional investigations. Plots were generated in $\mathrm{R}$ using ggplot2 (v3.3.3) ${ }^{132}$, EnhancedVolcano (v1.8.0) ${ }^{133}$, and ComplexHeatmap (v2.6.2) ${ }^{134}$. Storey's q value was utilized to control family-wise error rate ${ }^{135}$. Gene sets used for Gene Set Enrichment Analysis (GSEA) are listed in Supplementary Datasets 1 and 2. Sequencing data have been deposited at the Gene Expression Omnibus database under the GSE187394 accession.

\section{Metabolite analysis}

Polar metabolites were quantitatively profiled by a positive/negative ion-switching, targeted liquid chromatography tandem mass spectrometry (LC-MS/MS) based metabolomics platform using a 5500 QTRAP hybrid triple quadruple mass spectrometer (AB/SCIEX) via selected reaction monitoring (SRM) as described previously ${ }^{136}$. Briefly, Lin ${ }^{\text {neg }}$ bone marrow cells cultured with G-CSF and GM-CSF (40 $\mathrm{ng} / \mathrm{ml}$ each) for 48 hours using triplicate samples for each condition and sample type. After methanol extraction using $80 \%(\mathrm{v} / \mathrm{v})$ methanol $\left(-80^{\circ} \mathrm{C}\right)$ was carried out, pellets were lyophilized using a SpeedVac concentrator using no heat. $20 \mu \mathrm{L}$ of LC/MS grade water were added to resuspend each sample just before LC-MS/MS analysis and $5 \mu \mathrm{L}$ of sample were injected onto the autosampler of the LC system (Shimadzu) using an amide HILIC column (Waters). Once the SRM data for $~ 285$ metabolites were acquired, peaks 
were integrated using a software platform for peak area integration MultiQuant 2.1 (AB/SCIEX). Data analysis was performed using online MetaboAnalyst 3.0 software.

\section{Statistics}

Statistical analysis for comparison between two groups was determined by Student's $t$ test, while for comparison between three or more groups, ANOVA $\left(* \mathrm{p}\right.$ value $<0.05$, **p value $<0.01,{ }^{* * *}$ p value $<0.001$, **** p value $<0.0001)$ calculated with Prism software (GraphPad Software).

\section{Supplementary Materials}

Fig. S1. Gating strategy of $\mathrm{CD}^{+}$and $\mathrm{CD} 8^{+} \mathrm{T}$ cells in tumor draining lymph nodes (TDLN).

Fig. S2. Myeloid-specific but not T cell-specific SHP-2 depletion diminished MC17-51 tumor growth.

Fig. S3. Gating strategy of myeloid subsets in the spleen and tumor.

Fig. S4. Myeloid-specific SHP-2 depletion diminishes B16-F10 tumor growth and induces increase of M-MDSC.

Fig. S5. Myeloid-specific SHP-2 deletion does not affect the expression of co-inhibitory receptors in $\mathrm{CD}^{+}$or $\mathrm{CD} 8^{+} \mathrm{T}$ cells in tumor-bearing mice.

Fig. S6. Myeloid-specific SHP-2 deletion does not affect the expansion or activation of Treg cells.

Fig. S7. Diminished suppressive activity of M-MDSC isolated from Shp2 ${ }^{\mathrm{f} / \mathrm{f}}$ LysMCre tumor-bearing mice

Fig. S8. Heatmaps of pathway enrichment analysis genes in PMN-MDSC.

Fig. S9. Heatmaps of pathway enrichment analysis genes in TAMs. 
Fig. S10. Phagocytes generated from Shp $2^{\mathrm{f} / \mathrm{f}}$ LysMcre mice have enhanced metabolic activity.

Fig. S11. Pathway enrichment analysis on the top 500 up-regulated biological features in TAMs.

Fig. S12. Culture of bone marrow cells with GM-CSF+IL-3 induces upregulation of PD-1 and PD-L1 in $\operatorname{Lin}^{\text {neg }}$ and $\operatorname{Lin}^{\text {pos }}$ myelocytes.

Supplementary Table S1. List of DE genes in MDSCs identified by RNA-seq.

Supplementary Table S2. List of DE genes in TAMs identified by RNA-seq.

Supplementary Table S3. List of metabolites in bone marrow derived phagocytes.

Supplementary Table S4. List of antibodies used for flow cytometry.

Supplementary Dataset 1 . Gene sets used for GSEA in MDSCs.

Supplementary Dataset 2. Gene sets used for GSEA in TAMs. 


\section{Figure Legends}

Figure 1. SHP-2 deletion in the myeloid compartment diminishes B16-F10 melanoma tumor progression and suppressive function of MDSC. (A) Shp $2^{\mathrm{f} / \mathrm{f}}, \mathrm{Shp} 2^{\mathrm{f} / \mathrm{L}} \mathrm{LckCre}$ and $\mathrm{Shp} 2^{\mathrm{f} / \mathrm{f}} \mathrm{LysMCre}$ mice were inoculated with B16-F10 melanoma cells (1x10 cells/mouse) and tumor size was monitored. (B-C) Expression of CD44 was assessed in $\mathrm{CD}^{+} \mathrm{T}$ cells isolated from TDLNs. MFI $\pm \mathrm{SD}(\mathrm{B})$ and representative FACS histograms (C) are shown. (D) Diminished suppressive activity of MDSC isolated from Shp2 ${ }^{\mathrm{f} / \mathrm{L}}$ LysMCre tumor-bearing mice. GR ${ }^{+}$MDSCs cells were isolated from the spleens of Shp2 ${ }^{\mathrm{f} / \mathrm{f}}$, $\operatorname{Shp} 2{ }^{\mathrm{f} / \mathrm{L}}$ LckCre and $\mathrm{Shp} 2{ }^{\mathrm{f} / \mathrm{L}}$ LysMCre tumor-bearing mice and cultured at various ratios with OTI splenocytes $\left(2 \times 10^{5}\right.$ cells/well) stimulated with OVA257-264. Mean \pm SEM of cpm values are shown. (E-G) MDSC from Shp2 ${ }^{\mathrm{f} / \mathrm{f}}$ LysMCre tumor-bearing mice have diminished CD38 expression. Mean percentage \pm $\mathrm{SD}, \mathrm{MFI} \pm \mathrm{SD}(\mathrm{E})$, representative FACS histograms (F) and contour plots (G) of splenic MDSC are shown. Results are representative of two independent experiments with four to six mice per group. $(* \mathrm{p}<0.05, * *$ $\mathrm{p}<0.01, * * * \mathrm{p}<0.005)$

Figure 2. PD-1 blockade induces anti-tumor responses in Shp2 ${ }^{\mathrm{f} / \mathrm{f}}$ mice but does not further enhance anti-tumor responses of Shp2 $2^{\mathrm{f} / \mathrm{L}} \mathbf{L y s M C r e}$ mice. (A-C) Shp $2^{\mathrm{f} / \mathrm{f}}$ and Shp $2^{\mathrm{f} / \mathrm{f}} \mathrm{LysMCre}$ mice were inoculated with B16-F10 melanoma ( $3 \times 10^{5}$ cells/mouse) and were treated with anti-PD-1 blocking antibody or IgG2a control on days 9,11 and 13 after tumor inoculation. Tumor size was monitored starting on day 7 (A). Mice were euthanized on day 15 and tumor weight was measured (B). (D-G) Expression of CD38 is decreased by anti-PD-1 antibody treatment in tumor-bearing Shp2 $2^{\mathrm{fl} / \mathrm{fl}}$ but not Shp2 $2^{\mathrm{f} / \mathrm{f}}$ LysMCre mice. Mean percentage $\pm \mathrm{SD}(\mathrm{D}), \mathrm{MFI} \pm \mathrm{SD}(\mathrm{E})$, contour plots (F) and FACS histograms plots (G) of CD38 expression in splenic $\mathrm{CD} 11 \mathrm{~b}^{+} \mathrm{GR}-1^{+}$cells of tumor-bearing mice treated with anti-PD-1 blocking antibody or IgG2a. (H-I) CD44 expression is increased by anti-PD-1 antibody treatment in tumor-bearing Shp $2^{\text {fl/fl }}$ but not in 
Shp $2{ }^{\mathrm{f} / \mathrm{L}}$ LysMCre mice. MFI $\pm \mathrm{SD}$ of $\mathrm{CD} 44$ expression in $\mathrm{CD}^{+}$and $\mathrm{CD}^{+} \mathrm{T}$ cells of $\mathrm{TDLN}(\mathrm{H})$ and representative histograms of FACS analysis of $\mathrm{CD}^{+} \mathrm{T}$ cells $(\mathrm{I})$. (J-K) Increase of $\mathrm{CD}^{+}$and $\mathrm{CD} 8^{+} \mathrm{T}_{\mathrm{EF}}$ cells in TDLN of tumor-bearing $\operatorname{Shp} 2^{\mathrm{f} / \mathrm{f}}$ but not $\mathrm{Shp} 2^{\mathrm{f} / \mathrm{f}}$ LysMCre mice by anti-PD-1 antibody. Mean percentage $\pm \mathrm{SD}$ of $\mathrm{T}_{\mathrm{EF}}(\mathrm{J})$ and $\mathrm{T}_{\mathrm{CM}}(\mathrm{K}) \mathrm{CD} 4^{+}$and $\mathrm{CD} 8^{+}$cells in TDLNs of Shp2 $2^{\mathrm{f} / \mathrm{f}}$ and Shp $2^{\mathrm{f} / \mathrm{L}}$ LysMCre treated with anti-PD-1 blocking antibody or IgG2a. Results are representative of three separate experiments with four to six mice per group. $(* \mathrm{p}<0.05, * * \mathrm{p}<0.01, * * * \mathrm{p}<0.005)$.

Figure 3. Myeloid-specific SHP-2 deletion diminishes growth of MC17-51 tumor and alters monocytic tumor infiltration and $\mathbf{T}$ cell activation. (A-M) Shp $2^{\mathrm{f} / \mathrm{f}}$ and $\operatorname{Shp} 2^{\mathrm{f} / \mathrm{f}} \mathrm{LysMCre}$ mice were inoculated with MC17-51 cells and tumor size was monitored every two days starting on day 8 (A). Mice were euthanized on day 14 and tumor weight was measured (B). (C-D) Representative images of tumor bearing mice (C) and harvested tumors (D). (E-H) Increased monocytic cells in tumors of Shp2 $2^{\mathrm{f} / \mathrm{f}}$ LysMCre

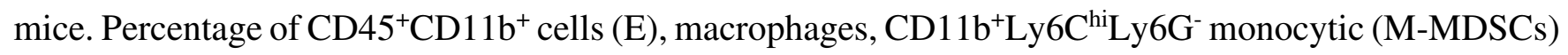
and $\mathrm{CD} 11 \mathrm{~b}^{+} \mathrm{Ly}_{6 \mathrm{C}}{ }^{\mathrm{lo}} \mathrm{Ly}_{6 \mathrm{G}}{ }^{+}$(PMN-MDSCs) (F) and representative pseudocolor plots of FACS analysis (G). The Ratio of M-MDSC:PMN-MDSC in the tumor site in Shp2 ${ }^{\mathrm{f} / \mathrm{f}}$ and $\mathrm{Shp} 2^{\mathrm{f} / \mathrm{f}}$ LysMCre tumor-bearing mice was calculated $(\mathrm{H})$. (I-N) Increased $\mathrm{CD}^{+}$and $\mathrm{CD}^{+} \mathrm{T}_{\mathrm{EF}}$ cell fractions and activation in TDLN and spleen of Shp2 $2^{\mathrm{f} / \mathrm{L}}$ LysMCre tumor-bearing mice. Mean percentage $\pm \mathrm{SD}$ of $\mathrm{T}_{\mathrm{EF}}$ and $\mathrm{T}_{\mathrm{CM}} \mathrm{CD} 4^{+}$and $\mathrm{CD} 8^{+} \mathrm{T}$ cells in TDLNs (I) and spleen (L) of Shp $2^{\mathrm{f} / \mathrm{f}}$ and Shp2 $2^{\mathrm{f} / \mathrm{f}}$ LysMCre tumor-bearing mice. Mean percentage \pm SD and $\mathrm{MFI} \pm \mathrm{SD}$ of $\mathrm{CD} 44$ expression in $\mathrm{CD}^{+}$and $\mathrm{CD}^{+}$cells in TDLN $(\mathrm{J})$ and spleen $(\mathrm{M})$ and representative histograms $(\mathrm{K}, \mathrm{N})$. Results are representative of four independent experiments with four to six mice per group. $(* \mathrm{p}<0.05, * * \mathrm{p}<0.01, * * * \mathrm{p}<0.005)$

Figure 4. SHP-2 deletion promotes myeloid cell differentiation to mature leukocytes with enhanced neutrophil-mediated immunity. (A) CD $11 b^{+} G R 1^{\text {hi }}$ cells were isolated from Shp $2^{\mathrm{f} / \mathrm{f}}$ and $\operatorname{Shp} 2^{\mathrm{f} / \mathrm{f}}$ LysMCre 
MC17-51 tumor-bearing mice and cultured at various ratios with OTI splenocytes stimulated with OVA257-264. Mean \pm SEM of cpm values is shown. (B-C) Mean percentage \pm SD, MFI \pm SD (B) and

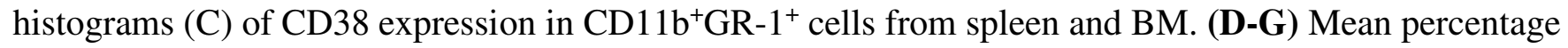
$\pm \mathrm{SD}$ and MFI \pm SD of MHC-II (D) and CD86 (E) expression in tumor infiltrating CD11b ${ }^{+} \mathrm{Ly}^{+} \mathrm{G}^{+}$and

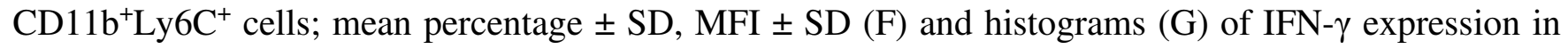
tumor infiltrating CD11b $\mathrm{Ly}_{6 \mathrm{G}}^{+}$and $\mathrm{CD} 11 \mathrm{~b}^{+} \mathrm{Ly} 6 \mathrm{C}^{+}$cells. Results are representative of three experiments with four to six mice per group. $(* \mathrm{p}<0.05, * * \mathrm{p}<0.01, * * * \mathrm{p}<0.005)$. (H-I) Differential expression of gene transcripts assessed by RNA-seq. Volcano plot $(\mathrm{H})$ of differentially expressed (DE) genes and heat map (I) of top 600 DE genes in PMN-MDSC cells isolated from spleens of Shp- $2^{\mathrm{f} / \mathrm{f}}$ and Shp- $2^{\mathrm{f} / \mathrm{f}} \mathrm{LysMCre}$ tumor-bearing mice (qValue $<0.05$ for all DEGs, $\log 2 \mathrm{FC}>0$ and $\log 2 \mathrm{FC}<0$ for up-regulated and downregulated genes, respectively). (J, K, L, M, N, R, S, T) Expression of the indicated genes by PMN-MDSC from Shp $2^{\mathrm{f} / \mathrm{f}}$ and Shp $2^{\mathrm{fl} / \mathrm{fl}}$ LysMCre tumor-bearing mice (data from RNA-seq dataset). (O) Bubble plot of significantly enriched pathways $(\mathrm{qValue}<0.1)$ in cells from Shp2 $2^{\mathrm{fl} / \mathrm{fl}}$ LysMCre mice sorted by GeneRatio. (P, Q) Heat maps of differentially expressed genes involved in myeloid cell differentiation (P) and neutrophil-mediated immunity $(\mathrm{Q})$ (qValue < 0.05$)$.

Figure 5. SHP-2 deletion increases monocyte and DC specification gene transcripts and imprints an effector differentiation program in tumor associated macrophages (TAMs). TAMs were isolated from Shp2 $2^{\mathrm{f} / \mathrm{f}}$ and $\mathrm{Shp} 2^{\mathrm{f} / \mathrm{f}}$ LysMCre MC17-51 tumor-bearing mice and differential expression of gene transcripts was assessed by RNA-seq. (A, B) Volcano plot (A) of DE genes and heat map (B) of the top $6500 \mathrm{DE}$ genes are shown (qValue $<0.05$ for all DEGs, $\log 2 \mathrm{FC}>0$ and $\log 2 \mathrm{FC}<0$ for up-regulated and down-regulated genes, respectively). (C, F, G, H, J, K, L, M, N) Expression of the IRF8 (C), Klf4 (F), Zeb2 (G), Batf3 (H), Tlr4 (J), Wdfy1 (K), Trem2 (L), Havrc2 (M) and Prdm1 (N) by TAMs from Shp2 $2^{\mathrm{f} / \mathrm{f}}$ and Shp $2^{\mathrm{f} / \mathrm{f}}$ LysMCre tumor-bearing mice (data from RNA-seq dataset). (D-E) Mean percentage \pm SD, $\mathrm{MFI} \pm \mathrm{SD}(\mathrm{D})$ and representative contour plots (E) of IRF8 expression; (U, V) Mean percentage \pm SD, 
$\mathrm{MFI} \pm \mathrm{SD}(\mathrm{U})$ and representative contour plots (V) of Ifn- $\gamma$ expression; (I, W) Mean percentage \pm SD and MFI \pm SD of CD86 (I) and IL-10 (W) in TAMs of tumor-bearing Shp2 $2^{\mathrm{f} / \mathrm{f}}$ and Shp2 ${ }^{\mathrm{f} / \mathrm{f}}$ LysMCre mice. (O) Bubble plot of significantly enriched pathways (qValue $<0.1$ ) in TAMs of Shp2 ${ }^{\mathrm{f} / \mathrm{f}}$ LysMCre mice sorted by GeneRatio. (P-S) Heat maps of differentially expressed genes related to macrophage differentiation (P), macrophage activation (Q), phagocytosis $(\mathrm{R})$ and TLR signaling $(\mathrm{S})$ are shown $(\mathrm{qValue}<0.05)$. $(\mathrm{T}$ X) Bubble plot of cytokine pathways (T) and metabolism pathways (X) significantly enriched in TAMs (qValue $<0.1)$ from Shp2 ${ }^{\mathrm{f} / \mathrm{f}}$ LysMCre mice sorted by GeneRatio.

Figure 6. Monocytes from tumor-bearing Shp2/f LysMCre obtain long-standing anti-tumor properties and transfer anti-tumor protection in naïve hosts. (A) GMPs and MDPs independently produce functionally distinct Ly6C hi inflammatory monocytes. GMPs produce classical and "neutrophillike' inflammatory monocytes, whereas MDPs produce classical and moDC-producing inflammatory monocytes. (B) Experimental schema: Shp2 ${ }^{\mathrm{f} / \mathrm{f}}$ LysMCre mice were implanted with MC17-51 tumors and nine days later, $\mathrm{CD} 45^{+} \mathrm{CD} 11 \mathrm{~b}^{+} \mathrm{Ly} 6 \mathrm{C}^{\mathrm{hi}} \mathrm{Ly}_{6 \mathrm{G}^{-}}$monocytes and $\mathrm{CD} 45^{+} \mathrm{CD} 11 \mathrm{~b}^{+} \mathrm{Ly} 6 \mathrm{C}^{-} \mathrm{Ly}_{6 \mathrm{G}^{+}}$neutrophils were collected from the bone marrow and implanted into naïve WT tumor bearing hosts together with MC1751 tumors. In parallel, a separate group of WT mice received only MC17-51 tumors without myeloid cells. (C-D) In all three groups tumor size was monitored every two days starting on day 7 (C). Mice were euthanized on day 15 and at termination tumor weight was measured; $n=10$ per group (D).

Figure 7. (A-C) Bone marrow cells from SHP-2 $2^{\mathrm{f} / \mathrm{f}}$ and SHP-2 $2^{\mathrm{f} / \mathrm{f}}$ LysMCre mice were cultured for $48 \mathrm{~h}$ in the presence of GM-CSF (10 ng/ml) and IL-3 (5 ng/ml). Cells were lysed and immunoprecipitation was performed with the indicated agarose-conjugated antibodies followed by SDS-PAGE and immunoblot with the indicated antibodies. (A) The abundance of phosphorylated HOXA10 (assessed by anti-pY blot) 
was normalized to immunoprecipitated HOXA10 and was expressed as fold change over the value obtained in SHP-2 ${ }^{\mathrm{f} / \mathrm{f}}$ cells, defined as 1 . Expression of actin in whole cell lysates was used as input. (B) The abundance of phosphorylated IRF8 (assessed by anti-pY blot) was normalized to immunoprecipitated IRF8 and was expressed as fold change over the value obtained in SHP-2 $2^{\mathrm{f} / \mathrm{f}}$ cells, defined as 1 . Expression of actin in whole cell lysates was examined as input. (C) The abundance of SHP-2 coprecipitated with PD-1 was normalized to immunoprecipitated PD-1 and was expressed as fold change over the value obtained in SHP-2 ${ }^{\mathrm{f} / \mathrm{f}}$ cells (defined as 1). (D-L) Bone marrow cells from wild type C57BL/6 WT mice were cultured for $48 \mathrm{~h}$ in media alone or in the presence of GM-CSF (10 ng/ml) and IL-3 (5 ng/ml). The fractions of Lin ${ }^{\text {neg }}$ and Lin $^{\text {pos }}$ cells were assessed (D) and expression of PD-1 and PD-L1 in Lin ${ }^{\text {neg }}$ and Lin $^{\text {pos }}$ cells was examined. MFI \pm SD and representative histograms of PD-1 and PD-L1 expression in $\operatorname{Lin}^{\text {neg }}(\mathrm{E}-\mathrm{H})$ and Lin ${ }^{\text {pos }}$ cells (I-L) are shown. (M) Bone marrow cells from wild type C57BL/6 WT mice cultured for $48 \mathrm{~h}$ in the presence of GM-CSF and IL-3 were rested for $3 \mathrm{~h}$ at $37^{\circ} \mathrm{C}$ in RPMI-1640 containing $10 \mathrm{mM}$ HEPES and then were either left untreated or stimulated with GM-CSF (40 ng/ml). At the indicated time points the reaction was stopped and lysates were prepared as described in Materials and Methods. Immunoprecipitation was performed with agarose-conjugated anti-PD-1 antibody followed by SDSPAGE and immunoblot with the indicated antibodies. The abundance of GMCSFR, Lyn, SHP-2 and PD1 phosphorylated at Y248 (pPD-1) coprecipitated with PD-1 (left panels) was normalized to immunoprecipitated PD-1 and was expressed as fold change over the value obtained in non-stimulated cells at the zero timepoint (defined as 1). Expression of indicated proteins in whole cell lysates was also examined (right panels). (N-O) Bone marrow cells from wild type C57BL/6 mice were cultured for 48h in the presence of GM-CSF (10 ng/ml) and IL-3 $(5 \mathrm{ng} / \mathrm{ml})$, alone or with anti-PD-L1 blocking antibody (MIH5) $(10 \mu \mathrm{g} / \mathrm{ml})$. Cells were lysed and immunoprecipitation was performed with indicated agaroseconjugated antibodies followed by SDS-PAGE and immunoblot with the indicated antibodies. (N) The abundance of phosphorylated HOXA10 (assessed by anti-pY blot) was normalized to immunoprecipitated HOXA10 and was expressed as fold change over the value obtained in cells cultured without PD-L1 
blocking antibody (defined as 1). Expression of actin in whole cell lysates was also examined as input. (O) The abundance of phosphorylated IRF8 (assessed by anti-pY blot) was normalized to immunoprecipitated IRF8 and was expressed as fold change over the value obtained in cells cultured without PD-L1 blocking antibody (defined as 1). Expression of actin in whole cell lysates was also examined as input. 


\section{Acknowledgments}

This work was supported by NIH/NCI grants CA238263-01, CA212605 and CA229784-01 (VAB).

\section{Author contribution}

AC, did the main body of the experiments and wrote sections of the manuscript; CC, IA, MAAM, LS, NMT, RP, did experiments; XLK and ISV did bioinformatics analysis; LB provided reagents: JA did metabolomics; NP did experiments, participated in the experimental design and wore sections of the manuscript; VAB designed the project, did experiments and wrote the manuscript. All authors read, edited and approved the manuscript.

\section{Competing interests}

VAB has patents on the PD-1 pathway licensed by Bristol-Myers Squibb, Roche, Merck, EMD-Serono, Boehringer Ingelheim, AstraZeneca, Novartis and Dako. The authors declare no other competing interests. 


\section{References}

1 Chen, D. S. \& Mellman, I. Elements of cancer immunity and the cancer-immune set point. Nature 541, 321-330, doi:10.1038/nature21349 (2017).

2 Baumeister, S. H., Freeman, G. J., Dranoff, G. \& Sharpe, A. H. Coinhibitory Pathways in Immunotherapy for Cancer. Annual review of immunology 34, 539-573, doi:10.1146/annurev-immunol-032414-112049 (2016).

3 Veglia, F., Sanseviero, E. \& Gabrilovich, D. I. Myeloid-derived suppressor cells in the era of increasing myeloid cell diversity. Nature reviews, doi:10.1038/s41577-020-00490-y (2021).

4 Broz, M. L. \& Krummel, M. F. The emerging understanding of myeloid cells as partners and targets in tumor rejection. Cancer immunology research 3, 313-319, doi:10.1158/2326-6066.CIR-15-0041 (2015).

5 Cassetta, L. \& Pollard, J. W. Tumor-associated macrophages. Curr Bio/ 30, R246-R248, doi:10.1016/j.cub.2020.01.031 (2020).

6 Lavin, Y. et al. Innate Immune Landscape in Early Lung Adenocarcinoma by Paired Single-Cell Analyses. Cell 169, 750-765 e717, doi:10.1016/j.cell.2017.04.014 (2017).

7 Molgora, M. et al. TREM2 Modulation Remodels the Tumor Myeloid Landscape Enhancing Anti-PD-1 Immunotherapy. Cell 182, 886-900 e817, doi:10.1016/j.cell.2020.07.013 (2020).

8 Bronte, V. et al. Recommendations for myeloid-derived suppressor cell nomenclature and characterization standards. Nature communications 7, 12150 (2016).

9 Iclozan, C., Antonia, S., Chiappori, A., Chen, D. T. \& Gabrilovich, D. Therapeutic regulation of myeloidderived suppressor cells and immune response to cancer vaccine in patients with extensive stage small cell lung cancer. Cancer Immunol Immunother 62, 909-918 (2013).

10 Meyer, C. et al. Frequencies of circulating MDSC correlate with clinical outcome of melanoma patients treated with ipilimumab. Cancer Immunol Immunother 63, 247-257 (2014).

11 Weber, R. et al. Myeloid-Derived Suppressor Cells Hinder the Anti-Cancer Activity of Immune Checkpoint Inhibitors. Frontiers in immunology 9, 1310, doi:10.3389/fimmu.2018.01310 (2018).

12 Jaynes, J. M. et al. Mannose receptor (CD206) activation in tumor-associated macrophages enhances adaptive and innate antitumor immune responses. Science translational medicine 12, doi:10.1126/scitranslmed.aax6337 (2020).

13 Iribarren, K. et al. Anticancer effects of anti-CD47 immunotherapy in vivo. Oncoimmunology 8, 1550619, doi:10.1080/2162402X.2018.1550619 (2019).

14 Hume, D. A. \& MacDonald, K. P. Therapeutic applications of macrophage colony-stimulating factor-1 (CSF-1) and antagonists of CSF-1 receptor (CSF-1R) signaling. Blood 119, 1810-1820, doi:10.1182/blood2011-09-379214 (2012).

15 Mantovani, A., Marchesi, F., Malesci, A., Laghi, L. \& Allavena, P. Tumour-associated macrophages as treatment targets in oncology. Nat Rev Clin Oncol 14, 399-416, doi:10.1038/nrclinonc.2016.217 (2017).

16 Bardhan, K., Anagnostou, T. \& Boussiotis, V. A. The PD1:PD-L1/2 Pathway from Discovery to Clinical Implementation. Frontiers in immunology 7, 550, doi:10.3389/fimmu.2016.00550 (2016).

17 Chemnitz, J. M., Parry, R. V., Nichols, K. E., June, C. H. \& Riley, J. L. SHP-1 and SHP-2 associate with immunoreceptor tyrosine-based switch motif of programmed death 1 upon primary human $\mathrm{T}$ cell stimulation, but only receptor ligation prevents T cell activation. J Immunol 173, 945-954 (2004).

18 Yokosuka, T. et al. Programmed cell death 1 forms negative costimulatory microclusters that directly inhibit T cell receptor signaling by recruiting phosphatase SHP2. The Journal of experimental medicine 209, 1201-1217 (2012).

19 Marasco, M. et al. Molecular mechanism of SHP2 activation by PD-1 stimulation. Sci Adv 6, eaay4458, doi:10.1126/sciadv.aay4458 (2020).

20 Patsoukis, N. et al. Interaction of SHP-2 SH2 domains with PD-1 ITSM induces PD-1 dimerization and SHP-2 activation. Commun Biol 3, 128, doi:10.1038/s42003-020-0845-0 (2020). 
21 Rota, G. et al. Shp-2 Is Dispensable for Establishing T Cell Exhaustion and for PD-1 Signaling In Vivo. Cell reports 23, 39-49, doi:10.1016/j.celrep.2018.03.026 (2018).

22 Zhang, T. et al. Loss of SHP-2 activity in CD4+ T cells promotes melanoma progression and metastasis. Sci Rep 3, 2845, doi:10.1038/srep02845 (2013).

23 Strauss, L. et al. Targeted deletion of PD-1 in myeloid cells induces antitumor immunity. Sci Immunol 5, doi:10.1126/sciimmunol.aay1863 (2020).

24 Liu, X. \& Qu, C. K. Protein Tyrosine Phosphatase SHP-2 (PTPN11) in Hematopoiesis and Leukemogenesis. J Signal Transduct 2011, 195239, doi:10.1155/2011/195239 (2011).

25 Tartaglia, M. et al. Somatic mutations in PTPN11 in juvenile myelomonocytic leukemia, myelodysplastic syndromes and acute myeloid leukemia. Nature genetics 34, 148-150, doi:10.1038/ng1156 (2003).

26 Lawrence, H. J., Sauvageau, G., Humphries, R. K. \& Largman, C. The role of HOX homeobox genes in normal and leukemic hematopoiesis. Stem Cells 14, 281-291, doi:10.1002/stem.140281 (1996).

27 Lindsey, S. et al. Activation of SHP2 protein-tyrosine phosphatase increases HoxA10-induced repression of the genes encoding gp91(PHOX) and p67(PHOX). The Journal of biological chemistry 282, 2237-2249, doi:10.1074/jbc.M608642200 (2007).

28 Lebert-Ghali, C. E. et al. Hoxa cluster genes determine the proliferative activity of adult mouse hematopoietic stem and progenitor cells. Blood 127, 87-90, doi:10.1182/blood-2015-02-626390 (2016).

29 Eklund, E. A. The role of HOX genes in malignant myeloid disease. Current opinion in hematology 14, 8589, doi:10.1097/MOH.0b013e32801684b6 (2007).

30 Eklund, E. A., Jalava, A. \& Kakar, R. Tyrosine phosphorylation of HoxA10 decreases DNA binding and transcriptional repression during interferon gamma -induced differentiation of myeloid leukemia cell lines. The Journal of biological chemistry 275, 20117-20126, doi:10.1074/jbc.M907915199 (2000).

31 Lindsey, S., Zhu, C., Lu, Y. F. \& Eklund, E. A. HoxA10 represses transcription of the gene encoding p67phox in phagocytic cells. J Immunol 175, 5269-5279, doi:10.4049/jimmunol.175.8.5269 (2005).

32 Becker, A. M. et al. IRF-8 extinguishes neutrophil production and promotes dendritic cell lineage commitment in both myeloid and lymphoid mouse progenitors. Blood 119, 2003-2012, doi:10.1182/blood-2011-06-364976 (2012).

33 Yanez, A., Ng, M. Y., Hassanzadeh-Kiabi, N. \& Goodridge, H. S. IRF8 acts in lineage-committed rather than oligopotent progenitors to control neutrophil vs monocyte production. Blood 125, 1452-1459, doi:10.1182/blood-2014-09-600833 (2015).

34 Netherby, C. S. et al. The Granulocyte Progenitor Stage Is a Key Target of IRF8-Mediated Regulation of Myeloid-Derived Suppressor Cell Production. J Immunol 198, 4129-4139, doi:10.4049/jimmunol.1601722 (2017).

35 Huang, W. et al. Leukemia-associated, constitutively active mutants of SHP2 protein tyrosine phosphatase inhibit NF1 transcriptional activation by the interferon consensus sequence binding protein. Molecular and cellular biology 26, 6311-6332, doi:10.1128/MCB.00036-06 (2006).

36 Zhu, C., Lindsey, S., Konieczna, I. \& Eklund, E. A. Constitutive activation of SHP2 protein tyrosine phosphatase inhibits ICSBP-induced transcription of the gene encoding gp91PHOX during myeloid differentiation. Journal of leukocyte biology 83, 680-691, doi:10.1189/jlb.0807514 (2008).

37 Zhang, E. E., Chapeau, E., Hagihara, K. \& Feng, G. S. Neuronal Shp2 tyrosine phosphatase controls energy balance and metabolism. Proceedings of the National Academy of Sciences of the United States of America 101, 16064-16069, doi:10.1073/pnas.0405041101 (2004).

38 Pan, D. et al. A major chromatin regulator determines resistance of tumor cells to T cell-mediated killing. Science (New York, N.Y 359, 770-775, doi:10.1126/science.aao1710 (2018).

39 Chamoto, K. et al. Mitochondrial activation chemicals synergize with surface receptor PD-1 blockade for T cell-dependent antitumor activity. Proceedings of the National Academy of Sciences of the United States of America 114, E761-E770 (2016).

40 Dammeijer, F. et al. The PD-1/PD-L1-Checkpoint Restrains T cell Immunity in Tumor-Draining Lymph Nodes. Cancer cell 38, 685-700 e688, doi:10.1016/j.ccell.2020.09.001 (2020). 
41 Karakasheva, T. A. et al. CD38+ M-MDSC expansion characterizes a subset of advanced colorectal cancer patients. JCl Insight 3, doi:10.1172/jci.insight.97022 (2018).

42 Strauss, L. et al. RORC1 Regulates Tumor-Promoting "Emergency" Granulo-Monocytopoiesis. Cancer cell 28, 253-269, doi:10.1016/j.ccell.2015.07.006 (2015).

43 Gabrilovich, D. I., Ostrand-Rosenberg, S. \& Bronte, V. Coordinated regulation of myeloid cells by tumours. Nature reviews 12, 253-268, doi:10.1038/nri3175 (2012).

44 Olsson, A. et al. Single-cell analysis of mixed-lineage states leading to a binary cell fate choice. Nature 537, 698-702, doi:10.1038/nature19348 (2016).

45 Yanez, A. et al. Granulocyte-Monocyte Progenitors and Monocyte-Dendritic Cell Progenitors Independently Produce Functionally Distinct Monocytes. Immunity 47, 890-902 e894, doi:10.1016/j.immuni.2017.10.021 (2017).

46 Fleith, R. C. et al. IFIT3 and IFIT2/3 promote IFIT1-mediated translation inhibition by enhancing binding to non-self RNA. Nucleic Acids Res 46, 5269-5285, doi:10.1093/nar/gky191 (2018).

47 Yuan, Y. et al. Targeting UBE4A Revives Viperin Protein in Epithelium to Enhance Host Antiviral Defense. Molecular cell 77, 734-747 e737, doi:10.1016/j.molcel.2019.11.003 (2020).

48 Zhong, Z. et al. New mitochondrial DNA synthesis enables NLRP3 inflammasome activation. Nature 560, 198-203, doi:10.1038/s41586-018-0372-z (2018).

49 Tang, Y. et al. Macrophage scavenger receptor 1 contributes to pathogenesis of fulminant hepatitis via neutrophil-mediated complement activation. J Hepatol 68, 733-743, doi:10.1016/j.jhep.2017.11.010 (2018).

50 Masucci, M. T., Minopoli, M., Del Vecchio, S. \& Carriero, M. V. The Emerging Role of Neutrophil Extracellular Traps (NETs) in Tumor Progression and Metastasis. Frontiers in immunology 11, 1749, doi:10.3389/fimmu.2020.01749 (2020).

51 Condamine, T. et al. Lectin-type oxidized LDL receptor-1 distinguishes population of human polymorphonuclear myeloid-derived suppressor cells in cancer patients. Sci Immunol 1, doi:10.1126/sciimmunol.aaf8943 (2016).

52 Yan, D., Wang, H. W., Bowman, R. L. \& Joyce, J. A. STAT3 and STAT6 Signaling Pathways Synergize to Promote Cathepsin Secretion from Macrophages via IRE1alpha Activation. Cell reports 16, 2914-2927, doi:10.1016/j.celrep.2016.08.035 (2016).

53 Bosteels, C. \& Scott, C. L. Transcriptional regulation of DC fate specification. Molecular immunology 121, 38-46, doi:10.1016/j.molimm.2020.02.021 (2020).

54 Sichien, D. et al. IRF8 Transcription Factor Controls Survival and Function of Terminally Differentiated Conventional and Plasmacytoid Dendritic Cells, Respectively. Immunity 45, 626-640, doi:10.1016/j.immuni.2016.08.013 (2016).

55 Grajales-Reyes, G. E. et al. Batf3 maintains autoactivation of Irf8 for commitment of a CD8alpha(+) conventional DC clonogenic progenitor. Nature immunology 16, 708-717, doi:10.1038/ni.3197 (2015).

56 Briseno, C. G. et al. Distinct Transcriptional Programs Control Cross-Priming in Classical and MonocyteDerived Dendritic Cells. Cell reports 15, 2462-2474, doi:10.1016/j.celrep.2016.05.025 (2016).

57 Gao, Y. et al. Single-Cell Analysis Reveals the Heterogeneity of Monocyte-Derived and Peripheral Type-2 Conventional Dendritic Cells. J Immunol 207, 837-848, doi:10.4049/jimmunol.2100094 (2021).

58 Menezes, S. et al. The Heterogeneity of Ly6C(hi) Monocytes Controls Their Differentiation into iNOS(+) Macrophages or Monocyte-Derived Dendritic Cells. Immunity 45, 1205-1218, doi:10.1016/j.immuni.2016.12.001 (2016).

59 Gajewski, T. F., Schreiber, H. \& Fu, Y. X. Innate and adaptive immune cells in the tumor microenvironment. Nature immunology 14, 1014-1022, doi:10.1038/ni.2703 (2013).

$60 \mathrm{Hu}, \mathrm{Y}$. H. et al. WDFY1 mediates TLR3/4 signaling by recruiting TRIF. EMBO reports 16, 447-455, doi:10.15252/embr.201439637 (2015).

61 Dixon, K. O. et al. TIM-3 restrains anti-tumour immunity by regulating inflammasome activation. Nature 595, 101-106, doi:10.1038/s41586-021-03626-9 (2021). 
62 Ulmert, I., Henriques-Oliveira, L., Pereira, C. F. \& Lahl, K. Mononuclear phagocyte regulation by the transcription factor Blimp-1 in health and disease. Immunology 161, 303-313, doi:10.1111/imm.13249 (2020).

63 Spranger, S., Bao, R. \& Gajewski, T. F. Melanoma-intrinsic beta-catenin signalling prevents anti-tumour immunity. Nature 523, 231-235, doi:10.1038/nature14404 (2015).

64 Urban-Wojciuk, Z. et al. The Role of TLRs in Anti-cancer Immunity and Tumor Rejection. Frontiers in immunology 10, 2388, doi:10.3389/fimmu.2019.02388 (2019).

65 Shime, H. et al. Toll-like receptor 3 signaling converts tumor-supporting myeloid cells to tumoricidal effectors. Proceedings of the National Academy of Sciences of the United States of America 109, 20662071, doi:10.1073/pnas.1113099109 (2012).

66 Han, C. et al. The AIM2 and NLRP3 inflammasomes trigger IL-1-mediated antitumor effects during radiation. Sci Immunol 6, doi:10.1126/sciimmunol.abc6998 (2021).

67 Kaneda, M. M. et al. PI3Kgamma is a molecular switch that controls immune suppression. Nature 539, 437-442, doi:10.1038/nature19834 (2016).

68 Guiducci, C., Vicari, A. P., Sangaletti, S., Trinchieri, G. \& Colombo, M. P. Redirecting in vivo elicited tumor infiltrating macrophages and dendritic cells towards tumor rejection. Cancer Res 65, 3437-3446, doi:10.1158/0008-5472.CAN-04-4262 (2005).

69 Lasagni, L. et al. An alternatively spliced variant of CXCR3 mediates the inhibition of endothelial cell growth induced by IP-10, Mig, and I-TAC, and acts as functional receptor for platelet factor 4 . The Journal of experimental medicine 197, 1537-1549, doi:10.1084/jem.20021897 (2003).

70 Tan, S. et al. Platelets enhance CD4+ central memory T cell responses via platelet factor 4-dependent mitochondrial biogenesis and cell proliferation. Platelets, 1-11, doi:10.1080/09537104.2021.1936479 (2021).

71 Xu, L. L., Warren, M. K., Rose, W. L., Gong, W. \& Wang, J. M. Human recombinant monocyte chemotactic protein and other $\mathrm{C}-\mathrm{C}$ chemokines bind and induce directional migration of dendritic cells in vitro. Journal of leukocyte biology 60, 365-371, doi:10.1002/jlb.60.3.365 (1996).

72 Carr, M. W., Roth, S. J., Luther, E., Rose, S. S. \& Springer, T. A. Monocyte chemoattractant protein 1 acts as a T-lymphocyte chemoattractant. Proceedings of the National Academy of Sciences of the United States of America 91, 3652-3656, doi:10.1073/pnas.91.9.3652 (1994).

73 Ramos, C. D. et al. MIP-1alpha[CCL3] acting on the CCR1 receptor mediates neutrophil migration in immune inflammation via sequential release of TNF-alpha and LTB4. Journal of leukocyte biology 78, 167-177, doi:10.1189/jlb.0404237 (2005).

74 Bystry, R. S., Aluvihare, V., Welch, K. A., Kallikourdis, M. \& Betz, A. G. B cells and professional APCs recruit regulatory T cells via CCL4. Nature immunology 2, 1126-1132, doi:10.1038/ni735 (2001).

75 Smith, D. F., Galkina, E., Ley, K. \& Huo, Y. GRO family chemokines are specialized for monocyte arrest from flow. Am J Physiol Heart Circ Physiol 289, H1976-1984, doi:10.1152/ajpheart.00153.2005 (2005).

76 Saeed, S. et al. Epigenetic programming of monocyte-to-macrophage differentiation and trained innate immunity. Science (New York, N.Y 345, 1251086, doi:10.1126/science.1251086 (2014).

77 Eruslanov, E. B. et al. Tumor-associated neutrophils stimulate T cell responses in early-stage human lung cancer. The Journal of clinical investigation 124, 5466-5480, doi:10.1172/JCI77053 (2014).

78 Singhal, S. et al. Origin and Role of a Subset of Tumor-Associated Neutrophils with Antigen-Presenting Cell Features in Early-Stage Human Lung Cancer. Cancer cell 30, 120-135, doi:10.1016/j.ccell.2016.06.001 (2016).

79 Kalafati, L. et al. Innate Immune Training of Granulopoiesis Promotes Anti-tumor Activity. Cell 183, 771785 e712, doi:10.1016/j.cell.2020.09.058 (2020).

80 Bekkering, S. et al. Metabolic Induction of Trained Immunity through the Mevalonate Pathway. Cell 172, 135-146 e139, doi:10.1016/j.cell.2017.11.025 (2018).

81 Westerterp, M. et al. Cholesterol Accumulation in Dendritic Cells Links the Inflammasome to Acquired Immunity. Cell metabolism 25, 1294-1304 e1296, doi:10.1016/j.cmet.2017.04.005 (2017). 
82 Edgar, L. et al. Hyperglycemia Induces Trained Immunity in Macrophages and Their Precursors and Promotes Atherosclerosis. Circulation 144, 961-982, doi:10.1161/CIRCULATIONAHA.120.046464 (2021).

83 Margueron, R. \& Reinberg, D. The Polycomb complex PRC2 and its mark in life. Nature 469, 343-349, doi:10.1038/nature09784 (2011).

84 Upadhyay, A. K., Horton, J. R., Zhang, X. \& Cheng, X. Coordinated methyl-lysine erasure: structural and functional linkage of a Jumonji demethylase domain and a reader domain. Curr Opin Struct Biol 21, 750760, doi:10.1016/j.sbi.2011.08.003 (2011).

85 Boussiotis, V. A. Molecular and Biochemical Aspects of the PD-1 Checkpoint Pathway. The New England journal of medicine 375, 1767-1778 (2016).

86 Hui, E. et al. T cell costimulatory receptor CD28 is a primary target for PD-1-mediated inhibition. Science (New York, N.Y 355, 1428-1433, doi:10.1126/science.aaf1292 (2017).

87 Hansen, G. et al. The structure of the GM-CSF receptor complex reveals a distinct mode of cytokine receptor activation. Cell 134, 496-507, doi:10.1016/j.cell.2008.05.053 (2008).

$88 \mathrm{Li}, \mathrm{Y}$., Shen, B. F., Karanes, C., Sensenbrenner, L. \& Chen, B. Association between Lyn protein tyrosine kinase (p53/56lyn) and the beta subunit of the granulocyte-macrophage colony-stimulating factor (GMCSF) receptors in a GM-CSF-dependent human megakaryocytic leukemia cell line (M-07e). J Immunol 155, 2165-2174 (1995).

89 Bardhan, K. et al. Phosphorylation of PD-1-Y248 is a marker of PD-1-mediated inhibitory function in human T cells. Sci Rep 9, 17252, doi:10.1038/s41598-019-53463-0 (2019).

90 Eklund, E. A. The role of HOX genes in myeloid leukemogenesis. Current opinion in hematology 13, 6773, doi:10.1097/01.moh.0000208467.63861.d6 (2006).

91 Yanez, A. \& Goodridge, H. S. Interferon regulatory factor 8 and the regulation of neutrophil, monocyte, and dendritic cell production. Current opinion in hematology 23, 11-17, doi:10.1097/MOH.0000000000000196 (2016).

92 Okazaki, T., Maeda, A., Nishimura, H., Kurosaki, T. \& Honjo, T. PD-1 immunoreceptor inhibits B cell receptor-mediated signaling by recruiting src homology 2-domain-containing tyrosine phosphatase 2 to phosphotyrosine. Proceedings of the National Academy of Sciences of the United States of America 98 , 13866-13871 (2001).

93 Ireland, J. M. \& Unanue, E. R. Autophagy in antigen-presenting cells results in presentation of citrullinated peptides to CD4 T cells. The Journal of experimental medicine 208, 2625-2632, doi:10.1084/jem.20110640 (2011).

94 Wolpe, S. D. et al. Identification and characterization of macrophage inflammatory protein 2. Proceedings of the National Academy of Sciences of the United States of America 86, 612-616, doi:10.1073/pnas.86.2.612 (1989).

95 Halama, N. et al. Tumoral Immune Cell Exploitation in Colorectal Cancer Metastases Can Be Targeted Effectively by Anti-CCR5 Therapy in Cancer Patients. Cancer cell 29, 587-601, doi:10.1016/j.ccell.2016.03.005 (2016).

96 Lim, S. Y., Yuzhalin, A. E., Gordon-Weeks, A. N. \& Muschel, R. J. Targeting the CCL2-CCR2 signaling axis in cancer metastasis. Oncotarget 7, 28697-28710, doi:10.18632/oncotarget.7376 (2016).

97 Hubel, P. et al. A protein-interaction network of interferon-stimulated genes extends the innate immune system landscape. Nature immunology 20, 493-502, doi:10.1038/s41590-019-0323-3 (2019).

98 Schlee, M. \& Hartmann, G. Discriminating self from non-self in nucleic acid sensing. Nature reviews 16, 566-580, doi:10.1038/nri.2016.78 (2016).

99 Reizis, B., Bunin, A., Ghosh, H. S., Lewis, K. L. \& Sisirak, V. Plasmacytoid dendritic cells: recent progress and open questions. Annual review of immunology 29, 163-183, doi:10.1146/annurev-immunol-031210101345 (2011).

100 Maeda, S. et al. Interferon-alpha acts on the S/G2/M phases to induce apoptosis in the G1 phase of an IFNAR2-expressing hepatocellular carcinoma cell line. The Journal of biological chemistry 289, 2378623795, doi:10.1074/jbc.M114.551879 (2014). 
101 Mullally, A. et al. Depletion of Jak2V617F myeloproliferative neoplasm-propagating stem cells by interferon-alpha in a murine model of polycythemia vera. Blood 121, 3692-3702, doi:10.1182/blood2012-05-432989 (2013).

102 Yang, Y. et al. Exploiting synthetic lethality for the therapy of ABC diffuse large B cell lymphoma. Cancer cell 21, 723-737, doi:10.1016/j.ccr.2012.05.024 (2012).

103 Vanpouille-Box, C., Demaria, S., Formenti, S. C. \& Galluzzi, L. Cytosolic DNA Sensing in Organismal Tumor Control. Cancer cell 34, 361-378, doi:10.1016/j.ccell.2018.05.013 (2018).

104 Lu, C. et al. DNA Sensing in Mismatch Repair-Deficient Tumor Cells Is Essential for Anti-tumor Immunity. Cancer cell 39, 96-108 e106, doi:10.1016/j.ccell.2020.11.006 (2021).

105 Mowat, C., Mosley, S. R., Namdar, A., Schiller, D. \& Baker, K. Anti-tumor immunity in mismatch repairdeficient colorectal cancers requires type I IFN-driven CCL5 and CXCL10. The Journal of experimental medicine 218, doi:10.1084/jem.20210108 (2021).

106 Chen, Y. N. et al. Allosteric inhibition of SHP2 phosphatase inhibits cancers driven by receptor tyrosine kinases. Nature 535, 148-152, doi:10.1038/nature18621 (2016).

107 Chen, D. et al. SHP-2 and PD-L1 Inhibition Combined with Radiotherapy Enhances Systemic Antitumor Effects in an Anti-PD-1-Resistant Model of Non-Small Cell Lung Cancer. Cancer immunology research 8, 883-894, doi:10.1158/2326-6066.CIR-19-0744 (2020).

108 Wang, Y. et al. SHP2 blockade enhances anti-tumor immunity via tumor cell intrinsic and extrinsic mechanisms. Sci Rep 11, 1399, doi:10.1038/s41598-021-80999-x (2021).

109 Quintana, E. et al. Allosteric Inhibition of SHP2 Stimulates Antitumor Immunity by Transforming the Immunosuppressive Environment. Cancer Res 80, 2889-2902, doi:10.1158/0008-5472.CAN-19-3038 (2020).

110 Xiao, P. et al. Myeloid-restricted ablation of Shp2 restrains melanoma growth by amplifying the reciprocal promotion of CXCL9 and IFN-gamma production in tumor microenvironment. Oncogene 37, 5088-5100, doi:10.1038/s41388-018-0337-6 (2018).

111 Hao, H. X. et al. Tumor Intrinsic Efficacy by SHP2 and RTK Inhibitors in KRAS-Mutant Cancers. Mol Cancer Ther 18, 2368-2380, doi:10.1158/1535-7163.MCT-19-0170 (2019).

112 Fedele, C. et al. SHP2 inhibition diminishes KRASG12C cycling and promotes tumor microenvironment remodeling. The Journal of experimental medicine 218, doi:10.1084/jem.20201414 (2021).

113 Chawla, A. et al. PPAR-gamma dependent and independent effects on macrophage-gene expression in lipid metabolism and inflammation. Nature medicine 7, 48-52 (2001).

114 Yoon, Y. S. et al. PPARgamma activation following apoptotic cell instillation promotes resolution of lung inflammation and fibrosis via regulation of efferocytosis and proresolving cytokines. Mucosal Immuno/ 8 , 1031-1046, doi:10.1038/mi.2014.130 (2015).

115 Sulciner, M. L. et al. Resolvins suppress tumor growth and enhance cancer therapy. The Journal of experimental medicine 215, 115-140, doi:10.1084/jem.20170681 (2018).

116 Cheng, S. C. et al. mTOR- and HIF-1alpha-mediated aerobic glycolysis as metabolic basis for trained immunity. Science (New York, N.Y 345, 1250684, doi:10.1126/science.1250684 (2014).

117 Bekkering, S. et al. In Vitro Experimental Model of Trained Innate Immunity in Human Primary Monocytes. Clin Vaccine Immunol 23, 926-933, doi:10.1128/CVI.00349-16 (2016).

118 Arts, R. J. W. et al. Immunometabolic Pathways in BCG-Induced Trained Immunity. Cell reports 17, 25622571, doi:10.1016/j.celrep.2016.11.011 (2016).

119 Stienstra, R., Netea-Maier, R. T., Riksen, N. P., Joosten, L. A. B. \& Netea, M. G. Specific and Complex Reprogramming of Cellular Metabolism in Myeloid Cells during Innate Immune Responses. Cell metabolism 26, 142-156 (2017).

120 Mitroulis, I. et al. Modulation of myelopoiesis progenitors is an integral component of trained immunity. Cell 172, 147-161 (2018).

121 Christ, A. et al. Western Diet Triggers NLRP3-Dependent Innate Immune Reprogramming. Cell 172, 162175 e114, doi:10.1016/j.cell.2017.12.013 (2018). 
122 Arts, R. j., Joosten, L. A. B. \& Netea, M. G. Immunometabolic cirtuits in trained immunity. Semin Immunol 28, 425-430 (2016).

123 Patsoukis, N. et al. Interaction of both SH2 domains of SHP-2 with a PD-1 homodimer is required for PD1-mediated inhibition of T cell responses. . J Immunol May 2017, 198 (1 Supplement) 124.11 (2017).

124 Martin, M. Cutadapt removes adapter sequences from high-throughput sequencing reads. EMBnet j. . EMBnet j. 17 (2011).

125 Andrews, S. A Quality Control Tool for High Throughput Sequence Data Available online at: http://www.bioinformatics.babraham.ac.uk/projects/fastac/. (2010).

126 Conesa, A. et al. A survey of best practices for RNA-seq data analysis. Genome Biol 17, 13, doi:10.1186/s13059-016-0881-8 (2016).

127 Dobin, A. et al. STAR: ultrafast universal RNA-seq aligner. Bioinformatics 29, 15-21, doi:10.1093/bioinformatics/bts635 (2013).

128 Yates, A. D. et al. Ensembl 2020. Nucleic Acids Res 48, D682-D688, doi:10.1093/nar/gkz966 (2020).

129 Liao, Y., Smyth, G. K. \& Shi, W. featureCounts: an efficient general purpose program for assigning sequence reads to genomic features. Bioinformatics 30, 923-930, doi:10.1093/bioinformatics/btt656 (2014).

130 Love, M. I., Huber, W. \& Anders, S. Moderated estimation of fold change and dispersion for RNA-seq data with DESeq2. Genome Biol 15, 550, doi:10.1186/s13059-014-0550-8 (2014).

131 Yu, G., Wang, L. G., Han, Y. \& He, Q. Y. clusterProfiler: an R package for comparing biological themes among gene clusters. OMICS 16, 284-287, doi:10.1089/omi.2011.0118 (2012).

132 Wickham, H. ggplot2: Elegant Graphics for Data Analysis. Springer-Verlag New York Available online at: https://ggplot2.tidyverse.org.

133 Blighe, K., Rana, S. \& Myles, L. EnhancedVolcano: Publication-ready volcano plots with enhanced colouring and labeling. $R$ package version 1.8.0. Available online at: https://github.com/kevinblighe/EnhancedVolcano. (2020).

$134 \mathrm{Gu}, \mathrm{Z}$., Eils, R. \& Schlesner, M. Complex heatmaps reveal patterns and correlations in multidimensional genomic data. Bioinformatics 32, 2847-2849, doi:10.1093/bioinformatics/btw313 (2016).

135 Storey, J. D. \& Tibshirani, R. Statistical significance for genomewide studies. Proceedings of the National Academy of Sciences of the United States of America 100, 9440-9445, doi:10.1073/pnas.1530509100 (2003).

136 Yuan, M., Breitkopf, S. B., Yang, X. \& Asara, J. M. A positive/negative ion-switching, targeted mass spectrometry-based metabolomics platform for bodily fluids, cells, and fresh and fixed tissue. Nat Protoc 7, 872-881, doi:10.1038/nprot.2012.024 (2012). 
A

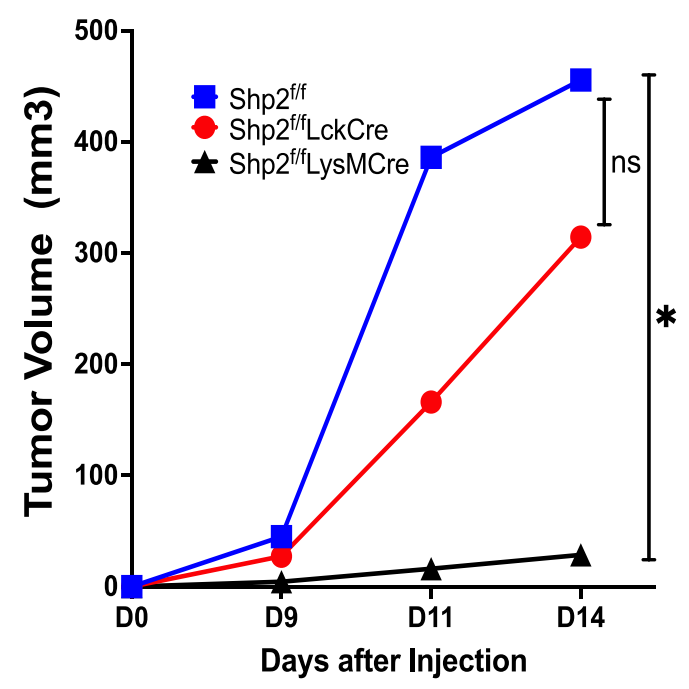

D

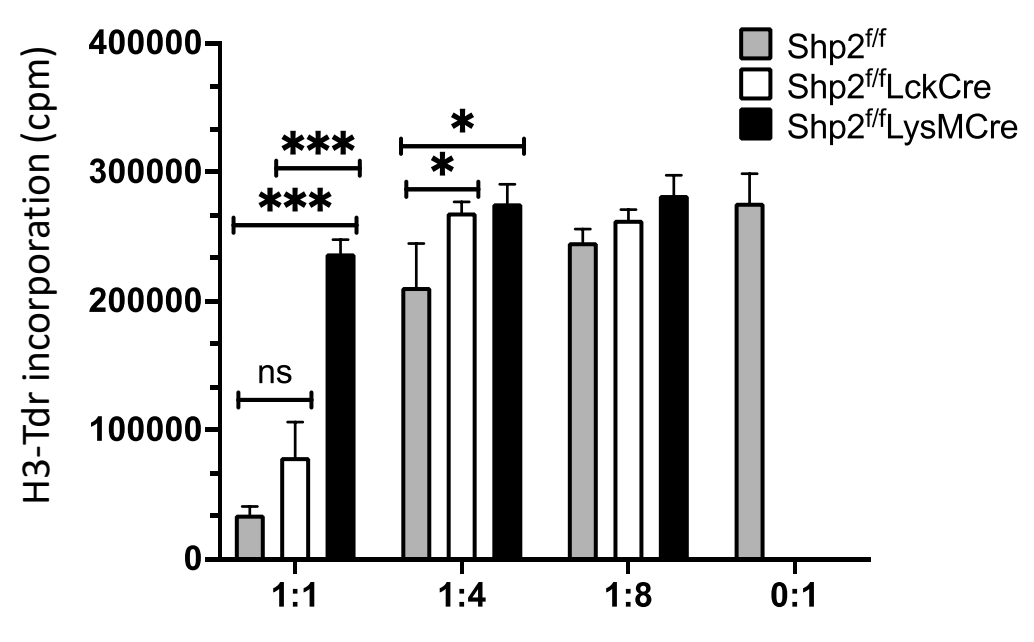

B

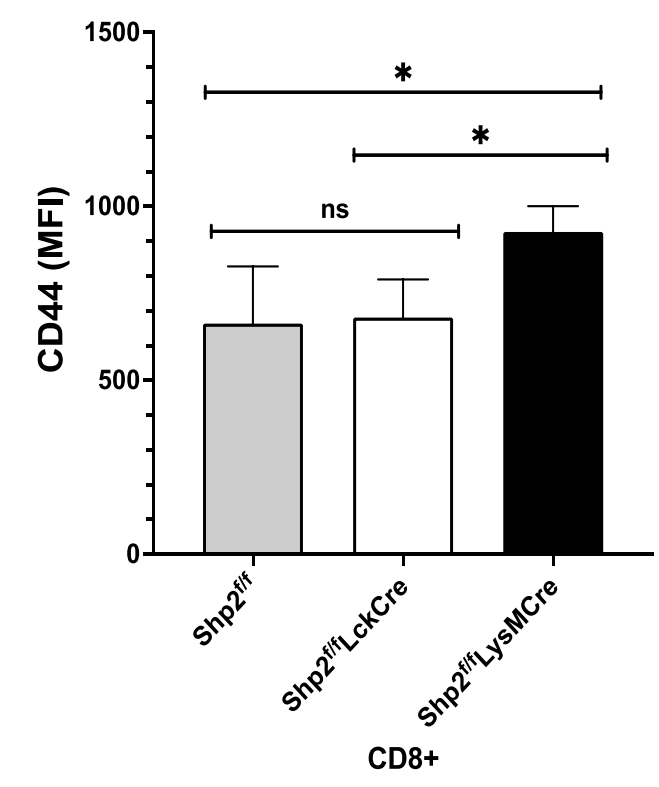

E

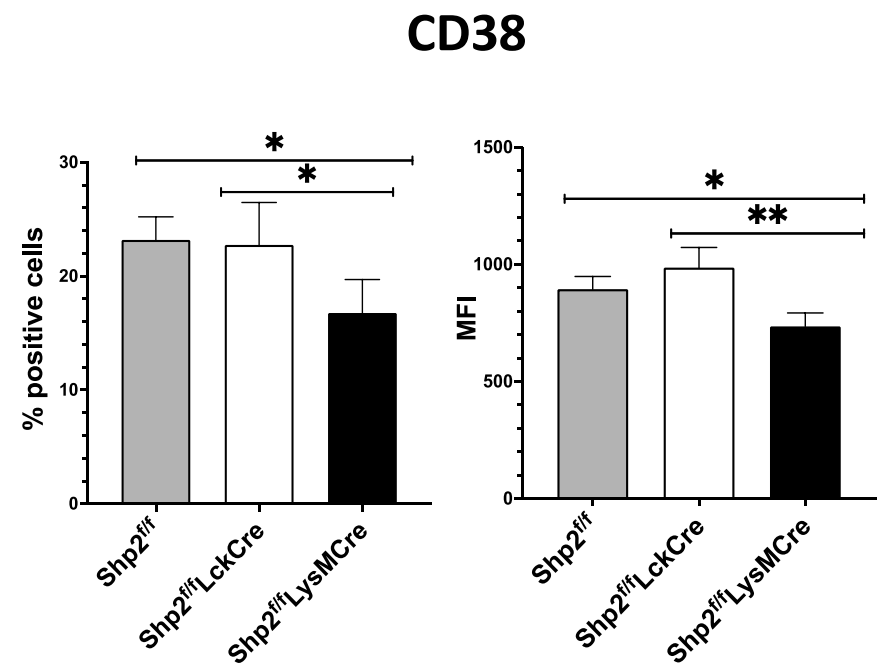

C

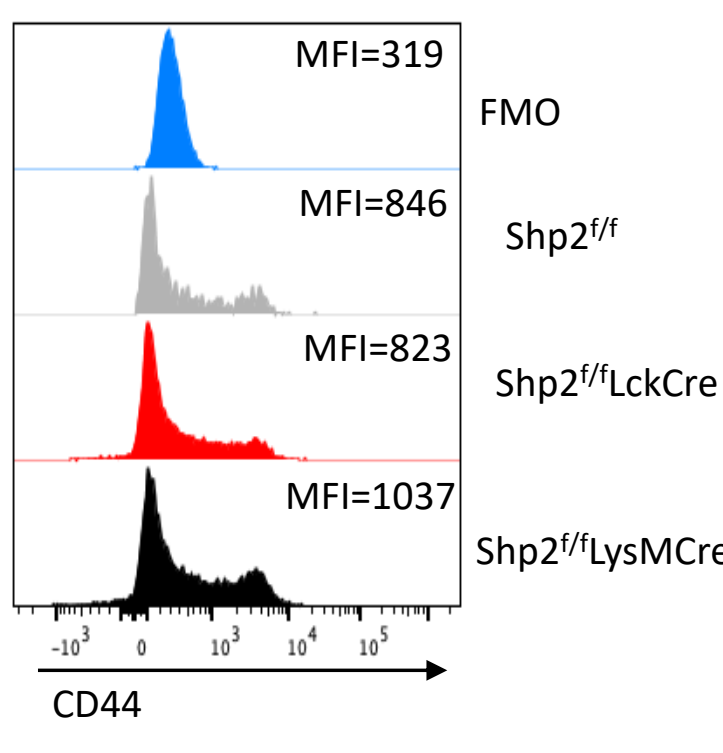

F

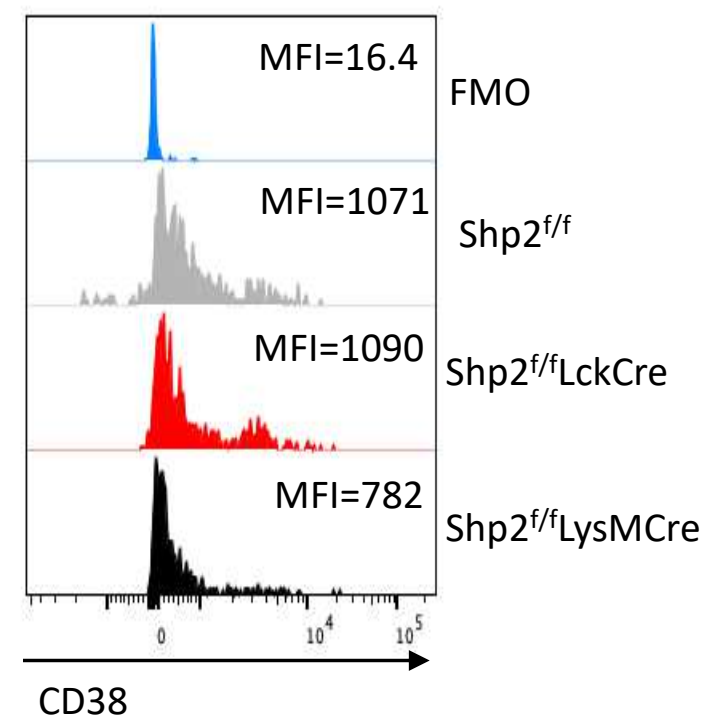

G
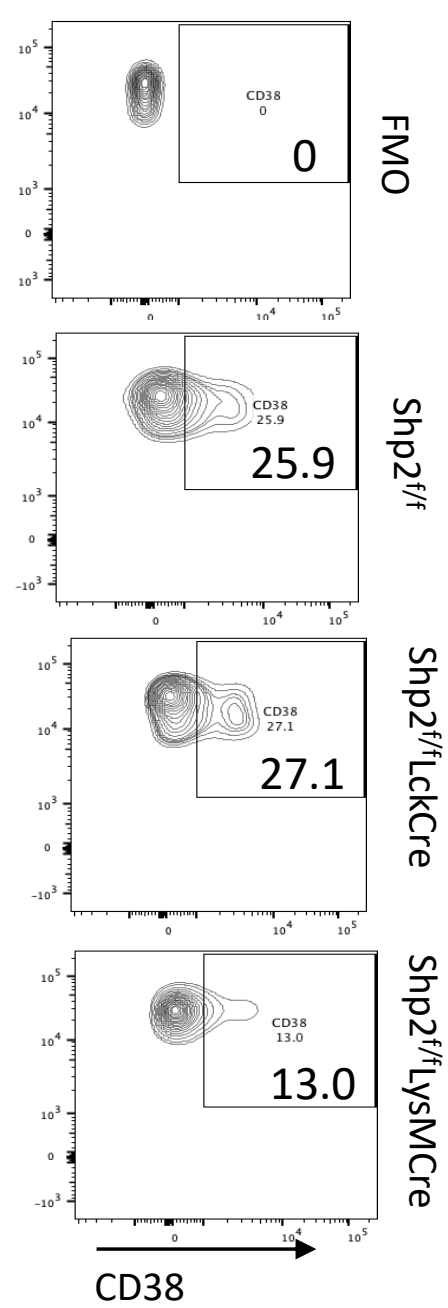

\section{Figure 1}


MC17-51
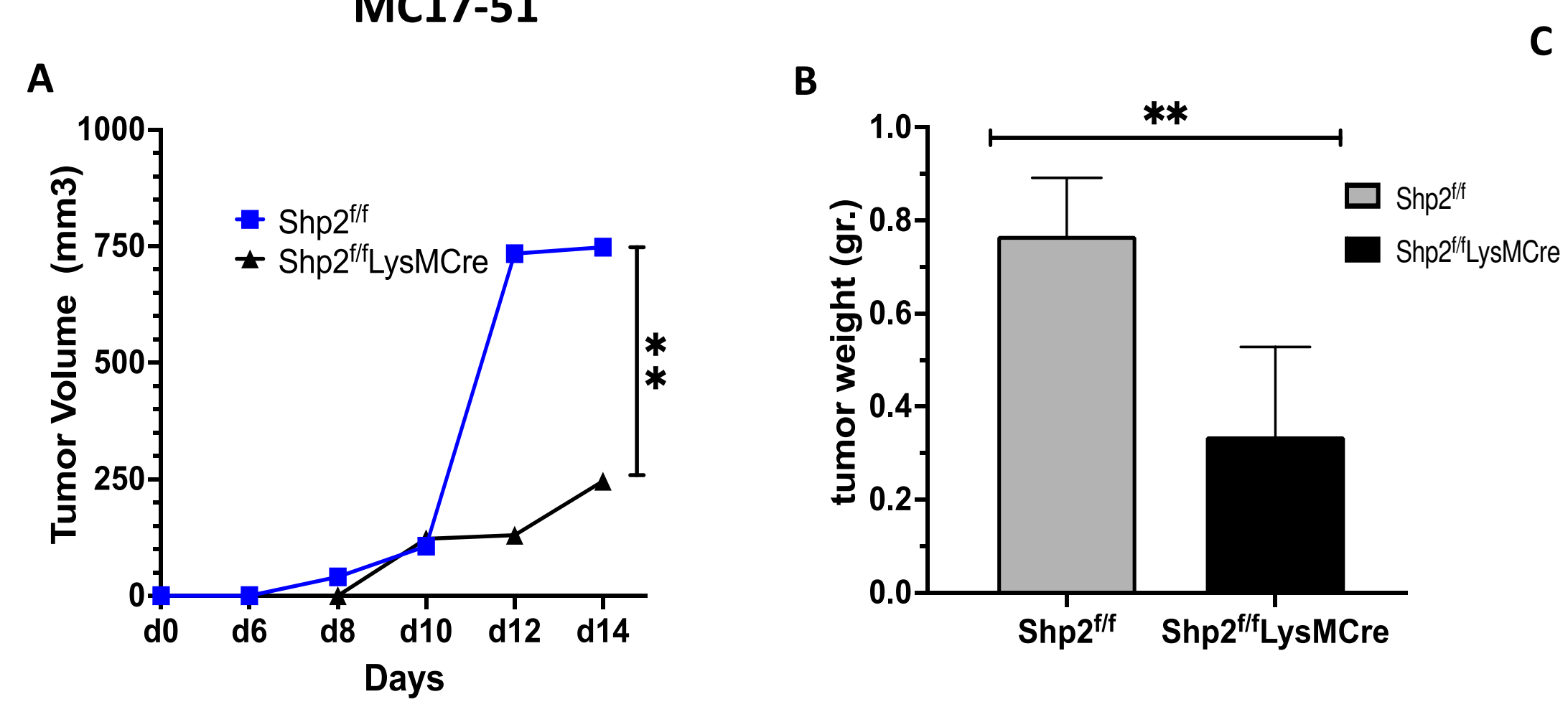

Tumor site

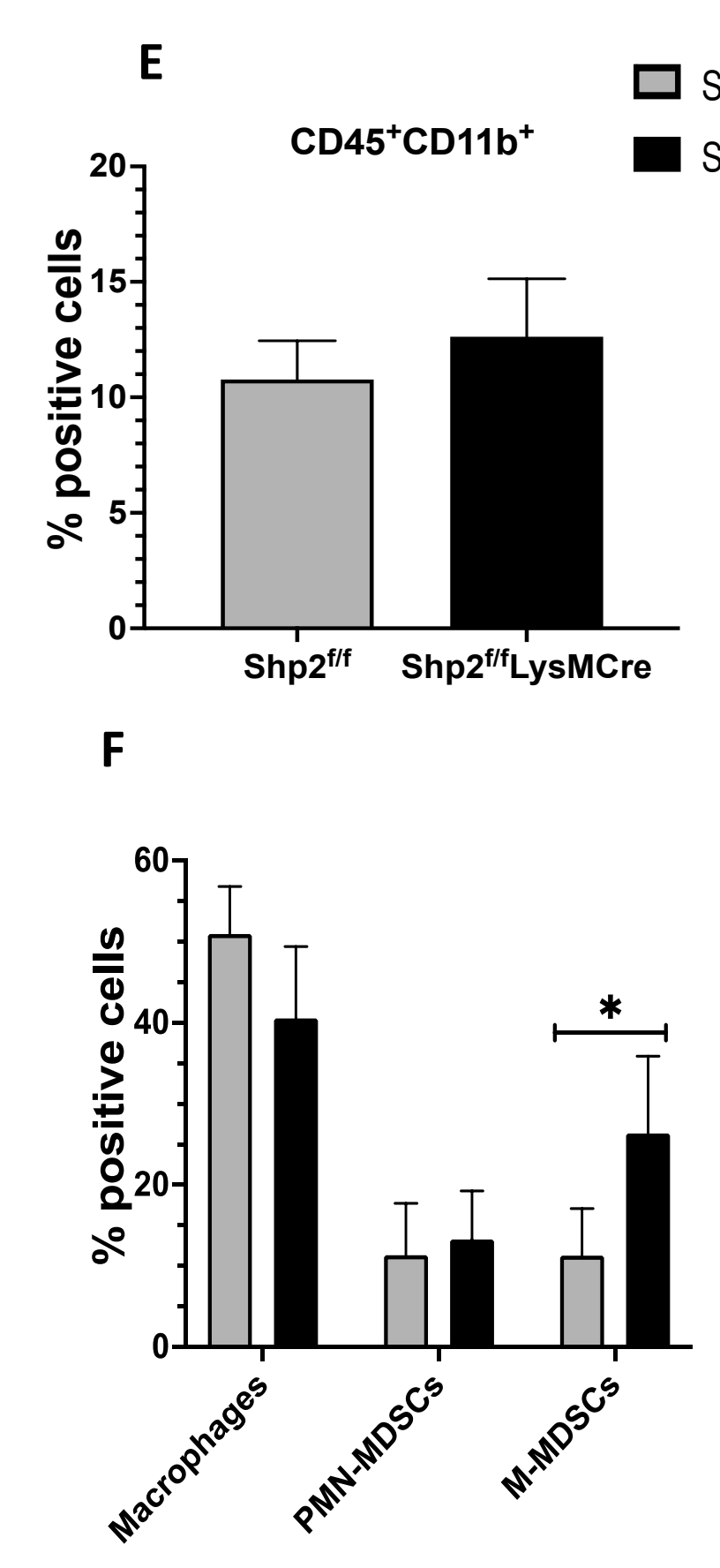

G

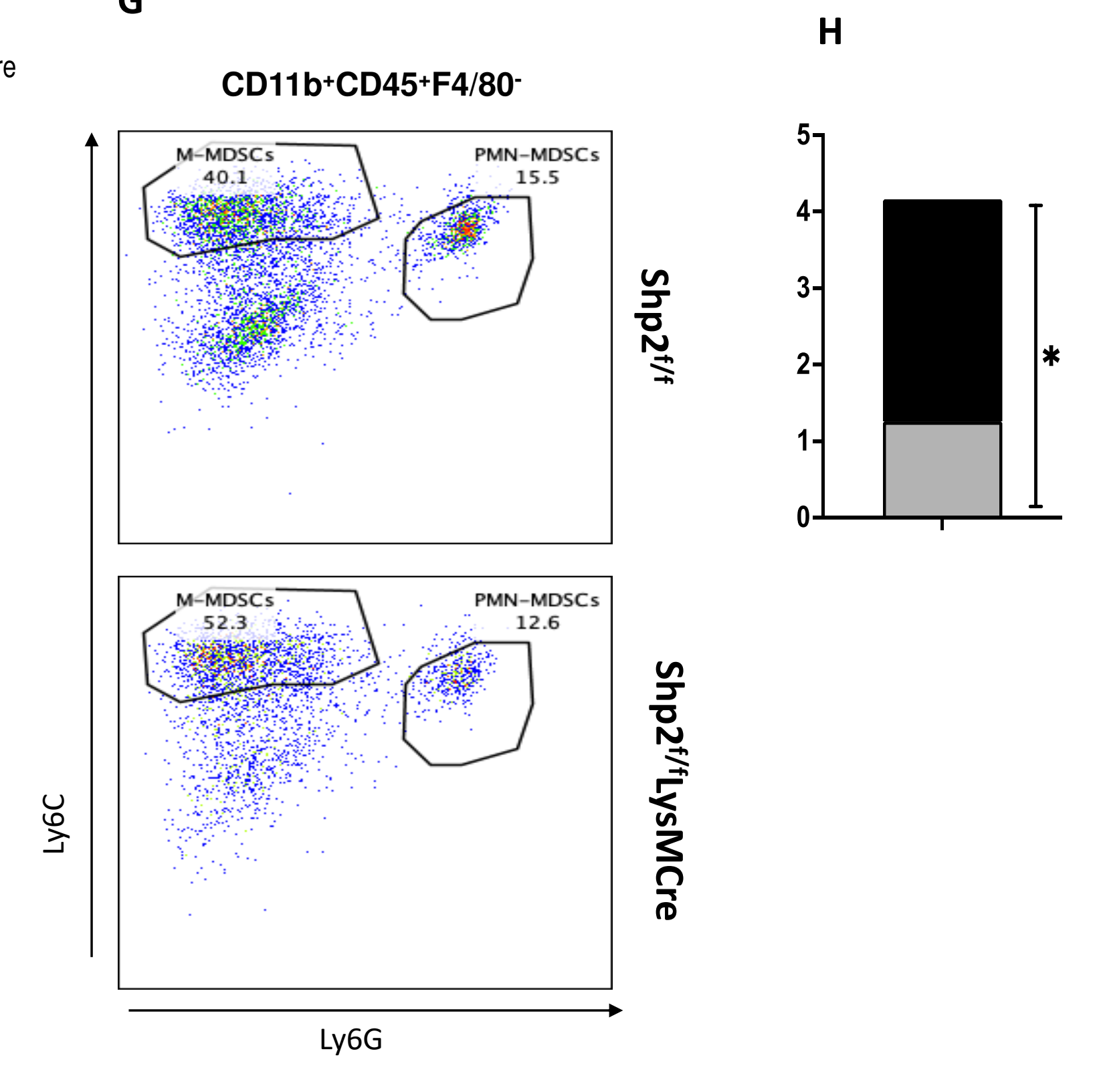

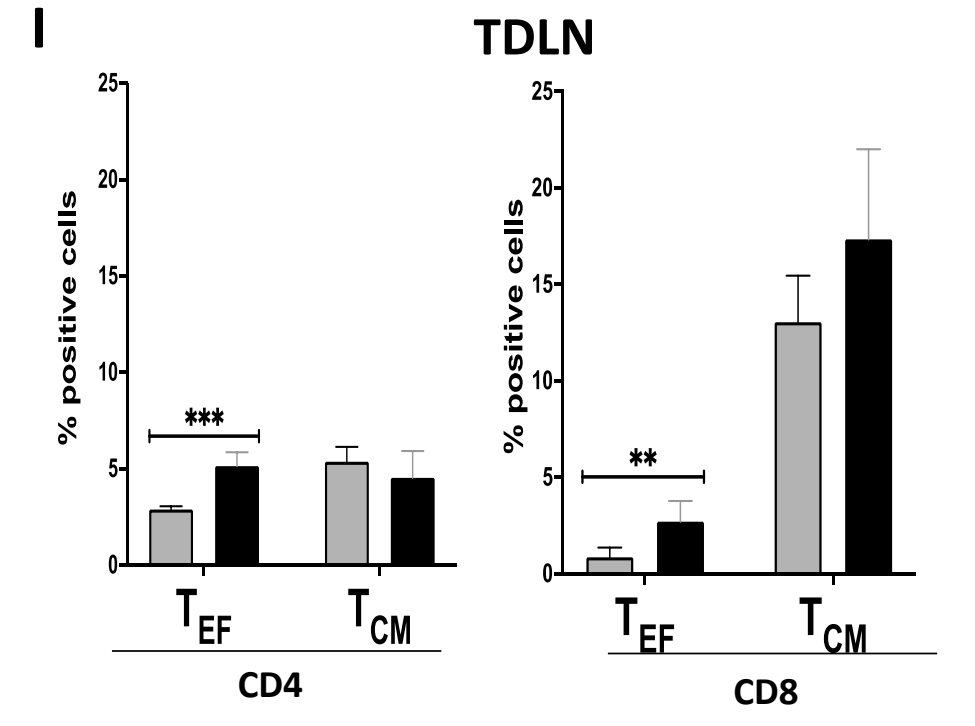
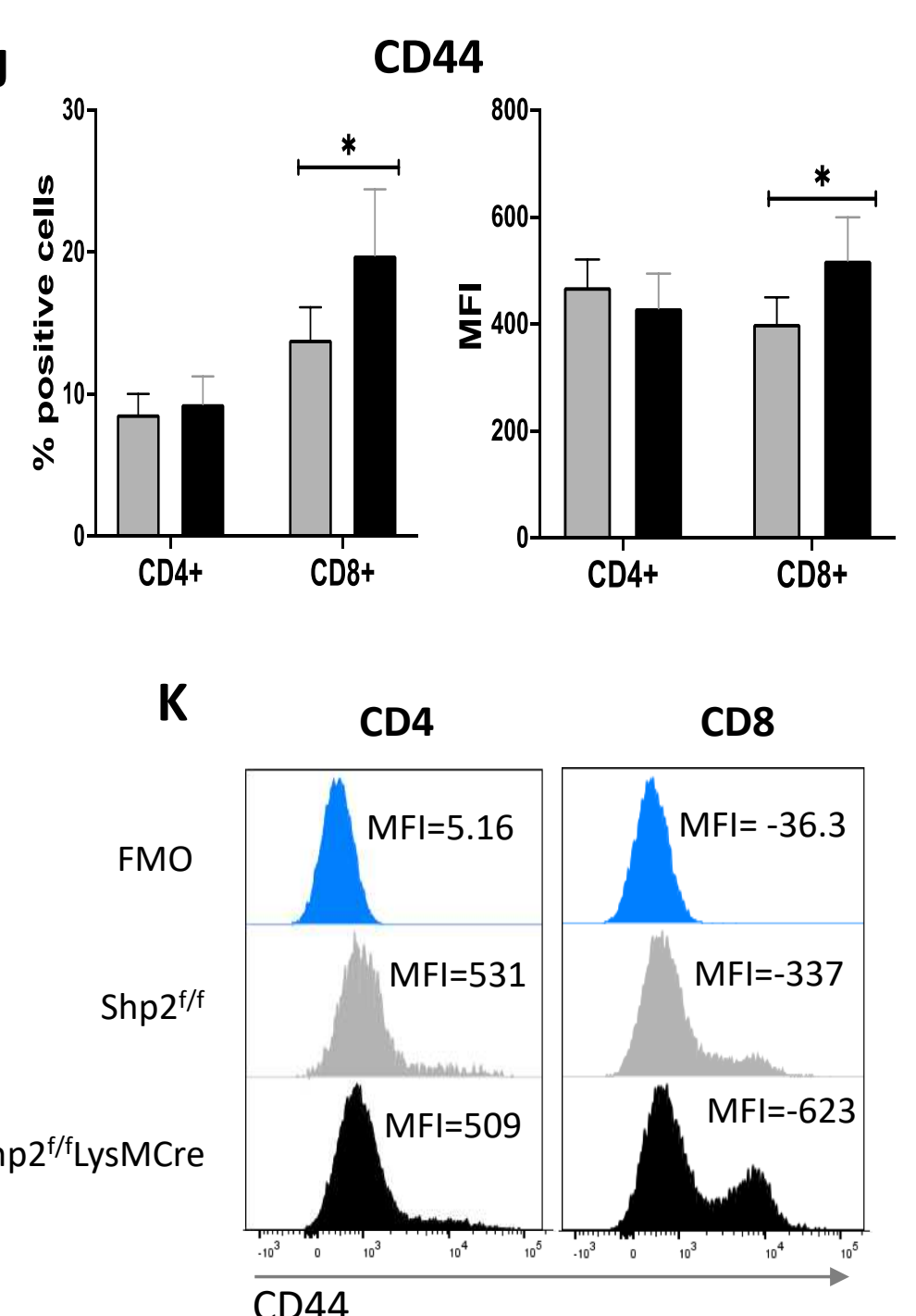

Shp2 ${ }^{f / f}$ LysMCre

$\operatorname{Shp} 2^{f / f}$
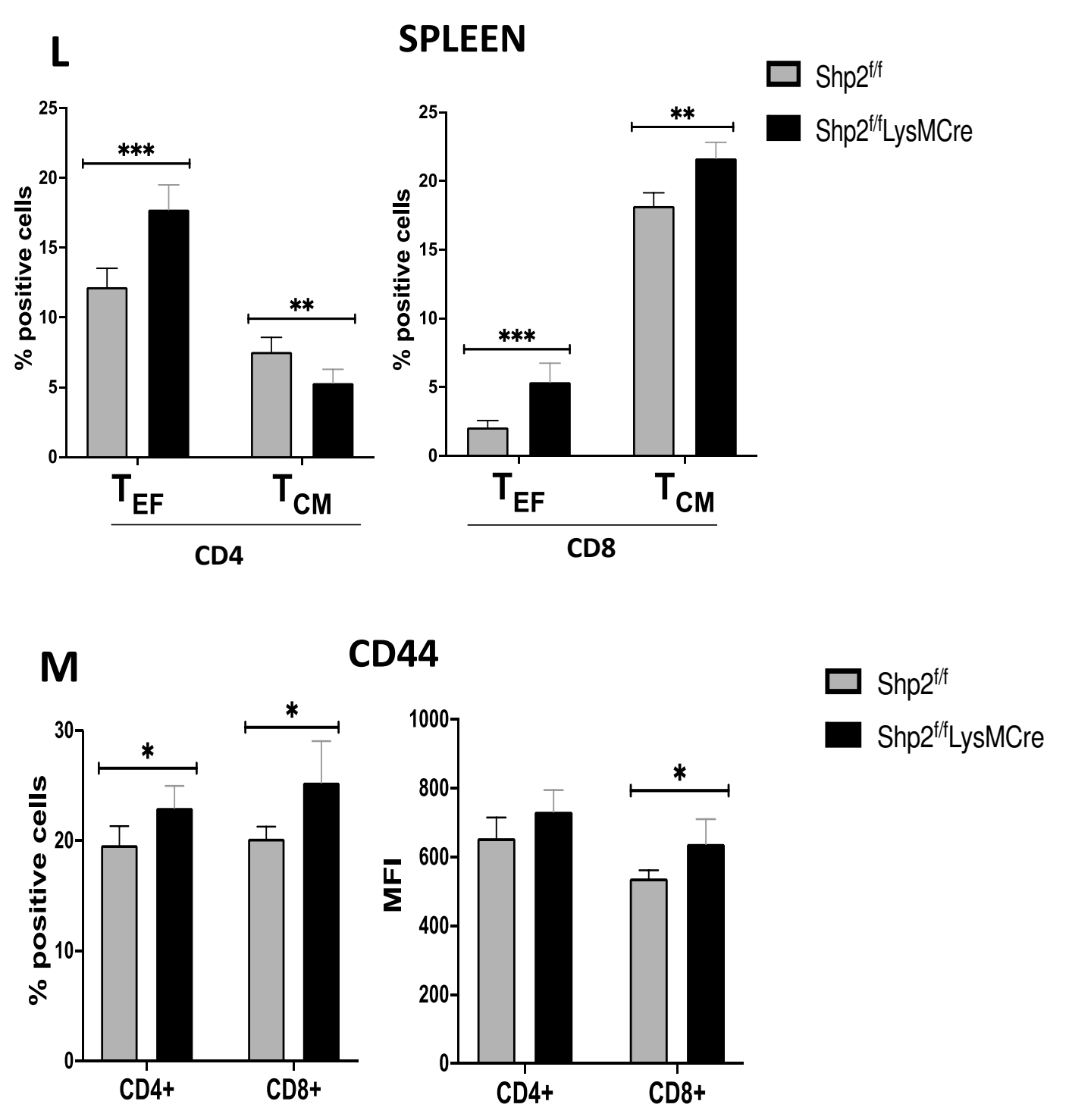

Shp2 2f/LySMCre 

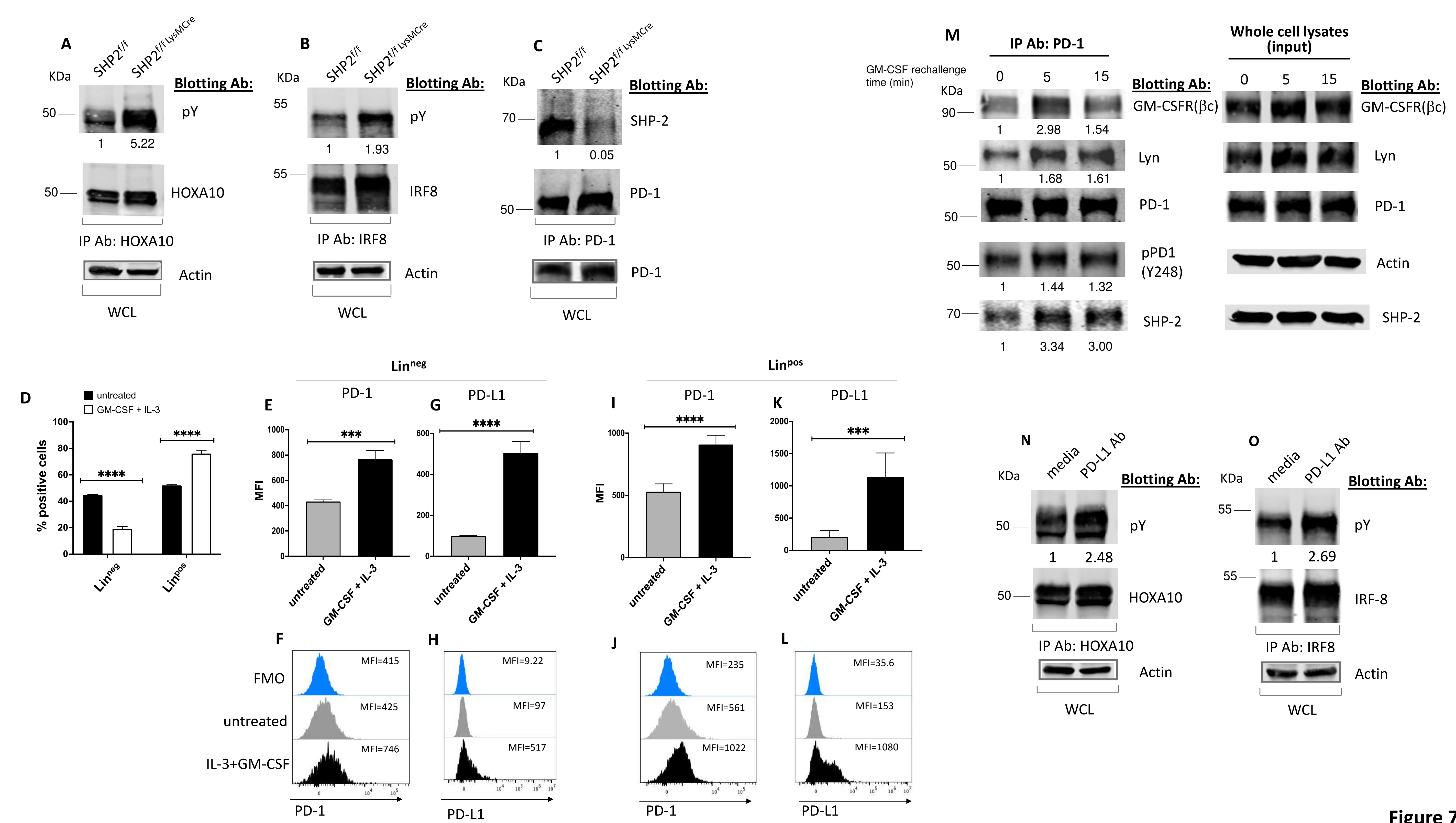

Figure 7 


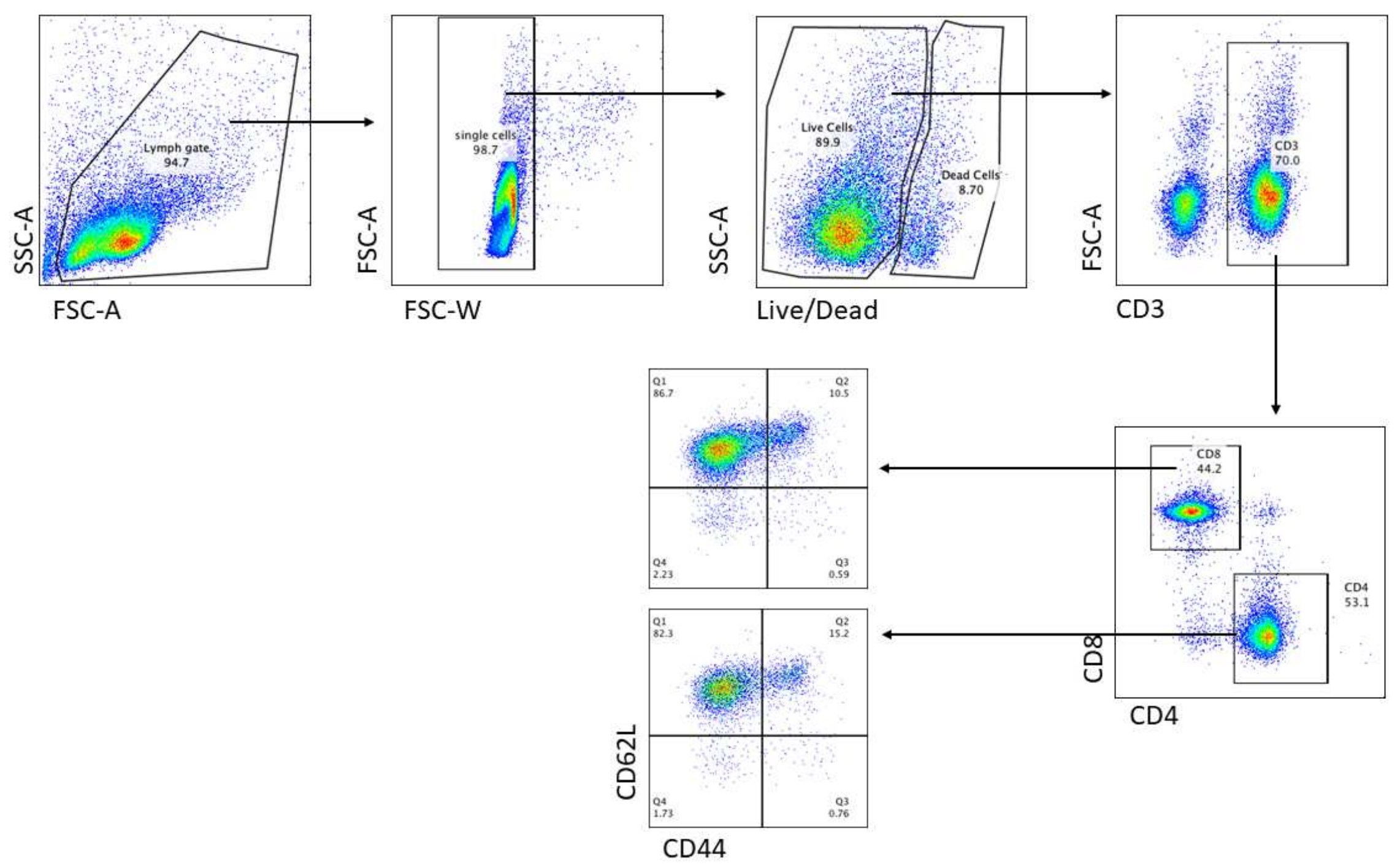

Fig. S1. Gating strategy of activated $\mathrm{CD4}^{+}$and $\mathrm{CD8}^{+} \mathrm{T}$ cells in tumor draining lymph nodes. After excluding doublets and dead cells, $\mathrm{CD}^{+} \mathrm{T}$ cells were divided into $\mathrm{CD} 4^{+}$and $\mathrm{CD} 8^{+} \mathrm{T}$ cells. Within the $\mathrm{CD} 4^{+}$and $\mathrm{CD} 8^{+}$gates, expression of $\mathrm{CD} 44$ and $\mathrm{CD} 62 \mathrm{~L}$ was assessed. 
A

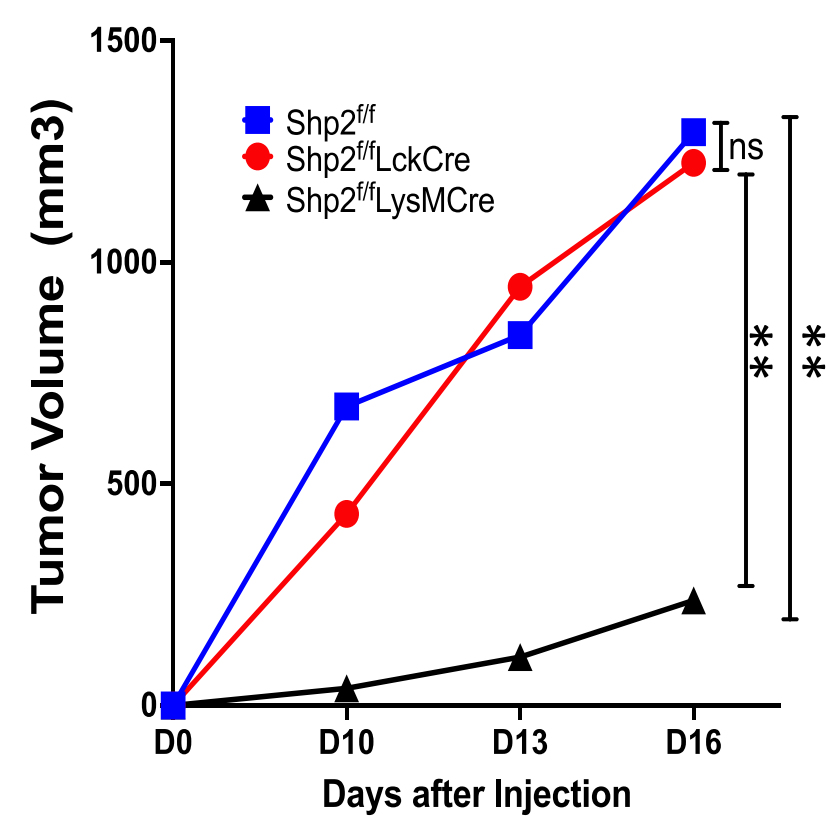

B

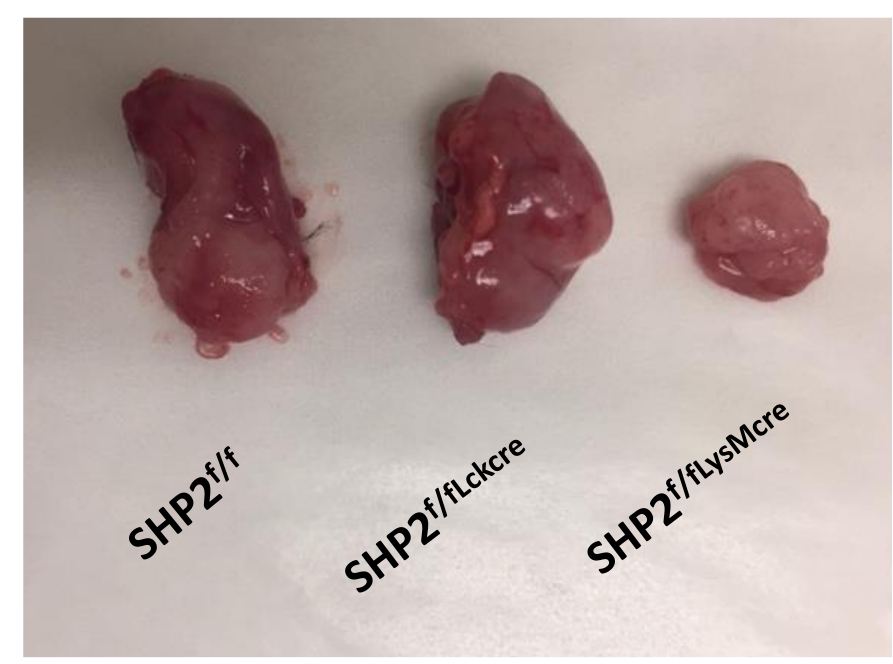

Fig. S2. Myeloid-specific but not T cell-specific SHP-2 depletion diminishes growth of MC17-51 tumor growth. (A) Shp2 ${ }^{\mathrm{f} / \mathrm{f}}$,

Shp2 ${ }^{\mathrm{f} / \mathrm{L}}$ Lckcre, and Shp2 $2^{\mathrm{f} / \mathrm{L}}$ LysMCre mice were inoculated with MC17-51 fibrosarcoma and tumor volume was monitored. Data shown are means of means of four to six mice per group from one representative of two experiments. (B) An image of the tumors isolated from each of the three strains at termination is shown. $(* * \mathrm{p}<0.01)$. 

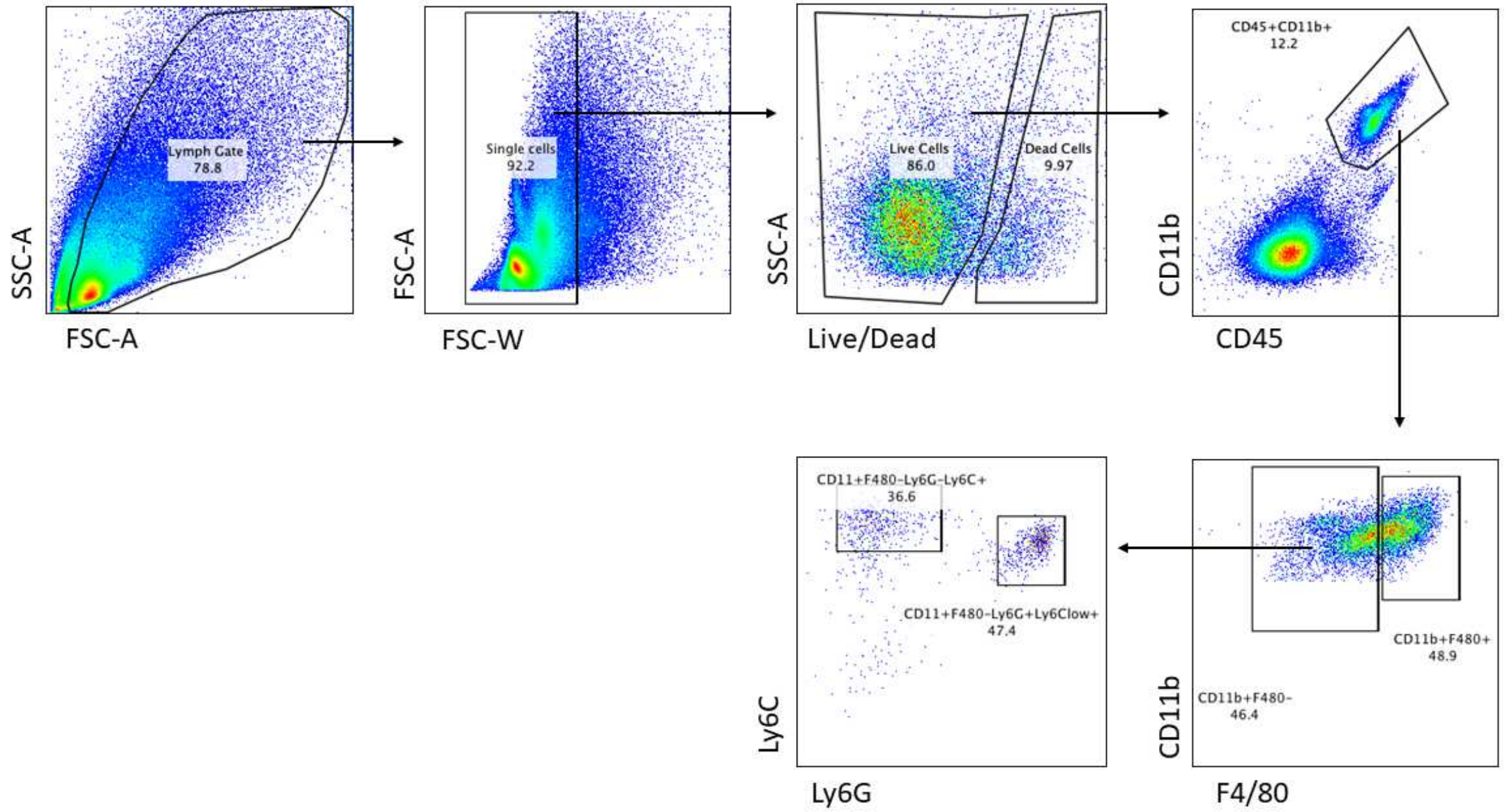

Fig. S3: Gating strategy of myeloid subsets in the spleen and tumor site. After excluding doublets and dead cells, myeloid cells were identified as $\mathrm{CD}^{2} 5^{+} \mathrm{CD} 11 \mathrm{~b}^{+}$. Within the myeloid cells, macrophages are identified as $\mathrm{CD} 11 \mathrm{~b}^{+} \mathrm{F} 4 / 80^{+}$, M-MDSCs as CD11 $\mathrm{b}^{+} \mathrm{F} 4 / 80^{+} \mathrm{Ly}^{6} \mathrm{G}^{-}$ Ly6C ${ }^{+}$and G-MDSCs as CD11b+F4/80+Ly6G+ $\mathrm{Ly}^{+} 6 \mathrm{C}^{\text {low }}$. 
A

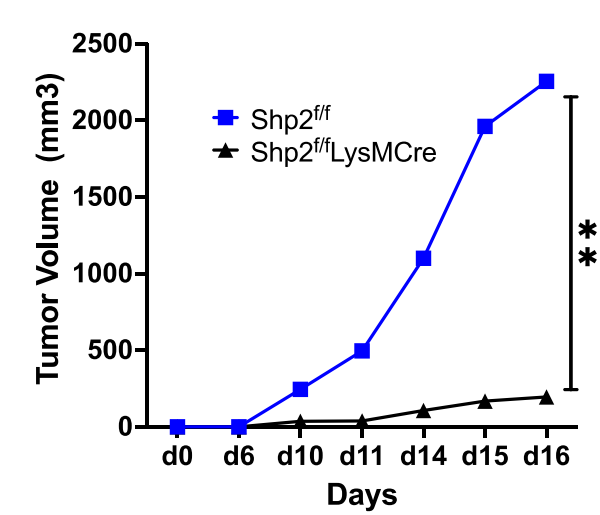

B

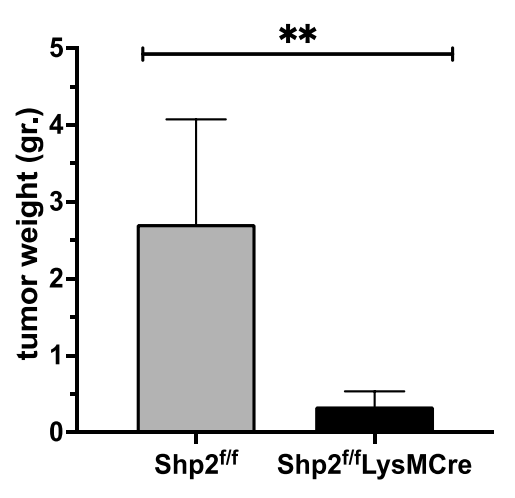

C

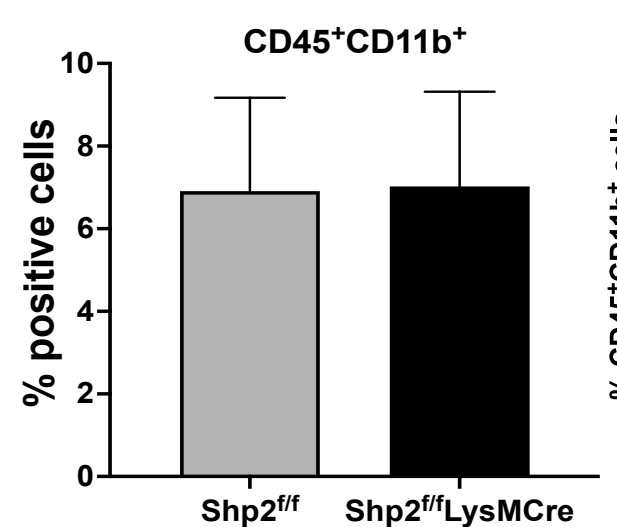

D

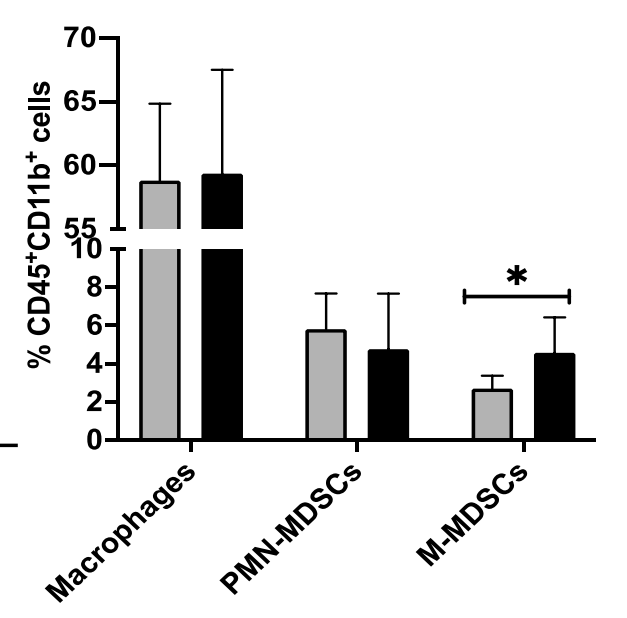

E

$\square \operatorname{Shp2}^{\text {fff }}$

- Shp2 $2^{f / t}$ LysMCre

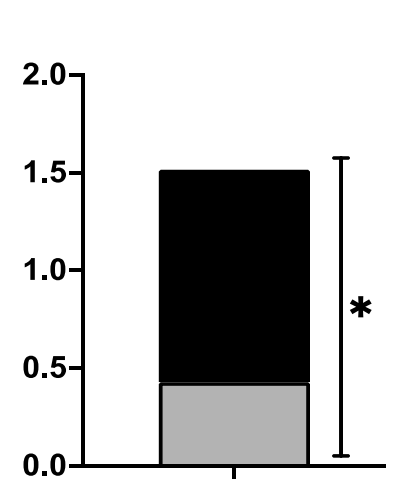

Fig. S4. Myeloid-specific SHP-2 deletion diminishes growth of B16-F10 tumor and induces increase of M-MDSC. (A-B) B16-F10 melanoma cells were inoculated in Shp2 ${ }^{\mathrm{f} / \mathrm{f}}$ and Shp2 ${ }^{\mathrm{f} / \mathrm{L}} \mathrm{LysMCre}$ mice as described in Methods. Tumor volume was monitored for 16 days (A), and tumor weight was measured at termination (B). (C-E) $\mathrm{CD}_{4} 5^{+} \mathrm{CD} 11 \mathrm{~b}^{+}$myeloid cells (C), CD11b $\mathrm{F} 4 / 80^{+}$macrophages, $\mathrm{CD} 11 \mathrm{~b}^{+} \mathrm{F} 4 / 80^{+} \mathrm{Ly} 6 \mathrm{G}^{+} \mathrm{Ly} 6 \mathrm{C}^{\text {low }}$ PMN-MDSCs and CD11b ${ }^{+} \mathrm{F} 4 / 80^{+}$Ly6G-Ly6C ${ }^{+} \mathrm{M}-\mathrm{MDSC}$ (D) in tumors were assessed. The ratio of MMDSC:PMN-MDSC in the tumor site in Shp2 ${ }^{\mathrm{f} / \mathrm{f}}$ and Shp2 $2^{\mathrm{f} / \mathrm{L}}$ LysMCre tumor-bearing mice was calculated (E). Representative plots and mean percentages \pm SD in Shp2 ${ }^{\mathrm{f} / \mathrm{f}}$ mice (grey bar) and Shp2 ${ }^{\mathrm{f} / \mathrm{L}}$ LysMCre (black bars) are shown. Results from one representative of four independent experiment with four to six mice per group are shown $\left({ }^{*} \mathrm{p}<0.05,{ }^{*} \mathrm{p}<0.01\right)$. 


\section{PD-1}

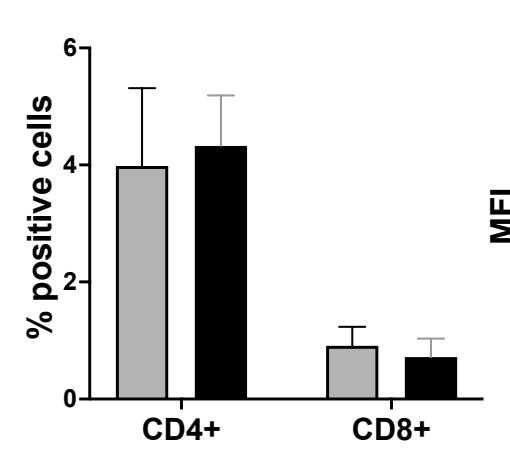

ICOS

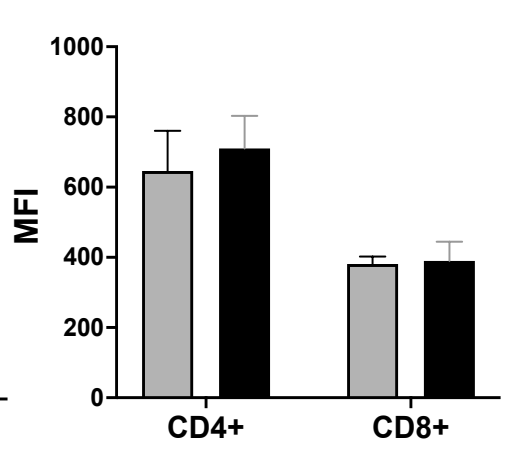

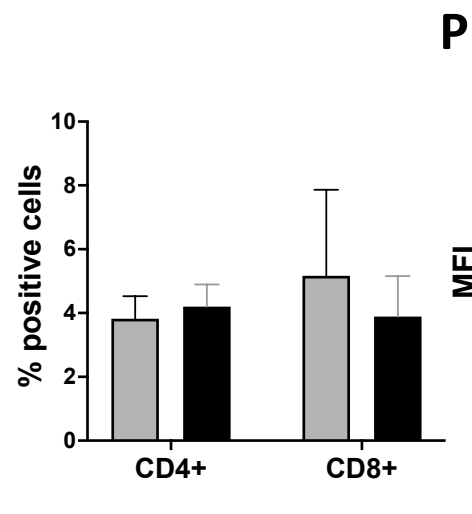

CTLA-4
PD-L1

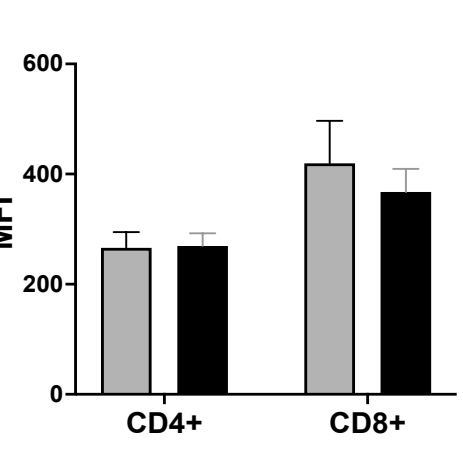

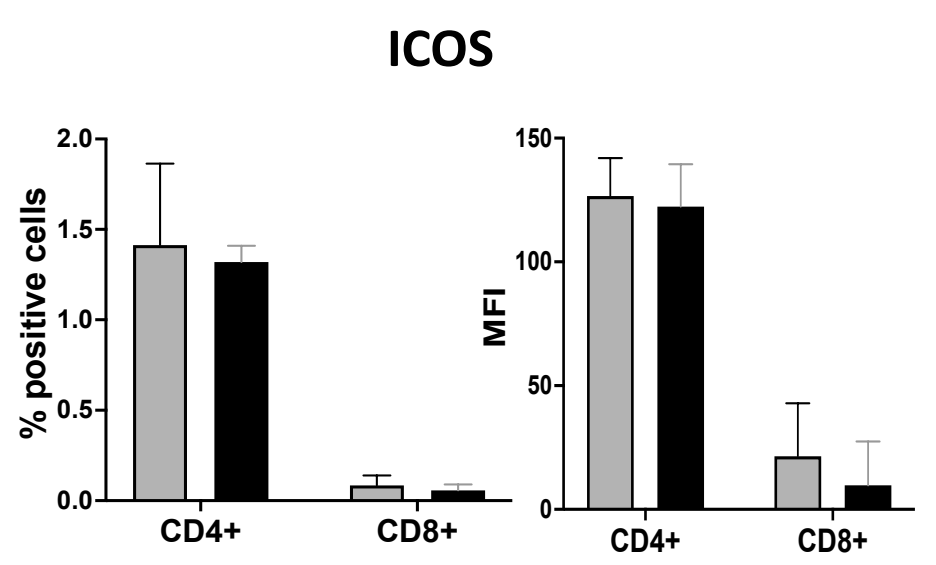
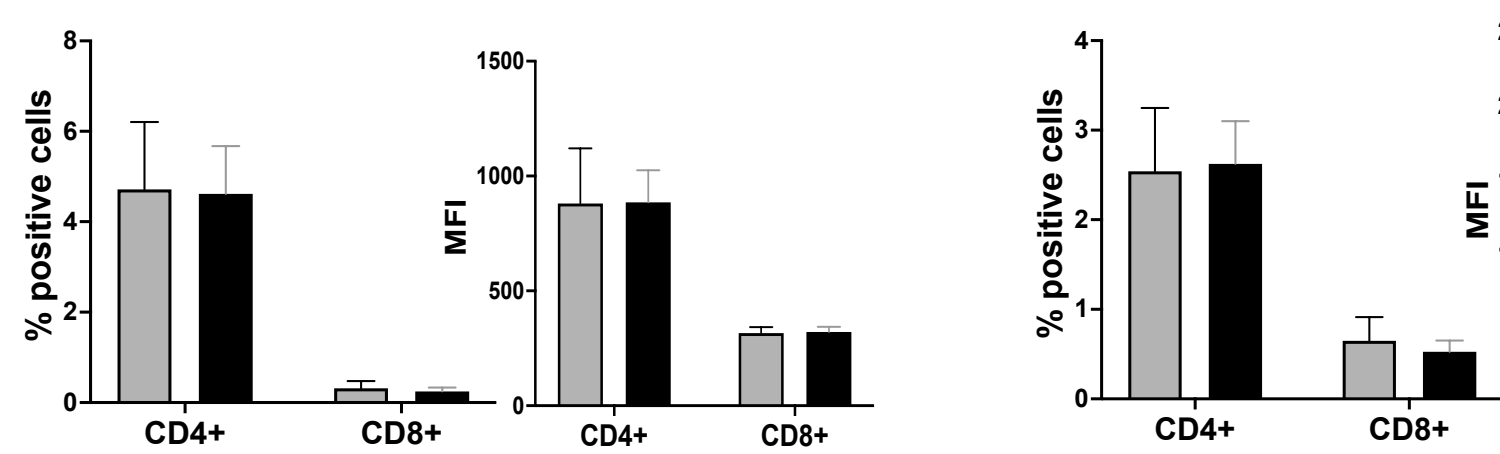

TIGIT

Fig. S5. Myeloid-specific SHP-2 deletion does not affect the expression of co-inhibitory receptors in $\mathrm{CD4}^{+}$or $\mathrm{CD8}^{+} \mathrm{T}^{\mathrm{Cells}}$. Expression of the indicated markers was assessed by flow cytometry in T cells isolated from TDLN of Shp2 ${ }^{\mathrm{f} / \mathrm{f}}$ and Shp2 $2^{\mathrm{f} / \mathrm{L}}$ LysMCre mice bearing MC1751 tumors. Representative plots and mean percentages \pm SD in Shp2 $2^{\mathrm{f} / \mathrm{f}}$ mice (grey bar) and Shp2 $2^{\mathrm{f} / \mathrm{L}}$ LysMCre (black bars) are shown. Results from one representative of four independent experiment with four to six mice per group are shown. 


\section{Treg}

A

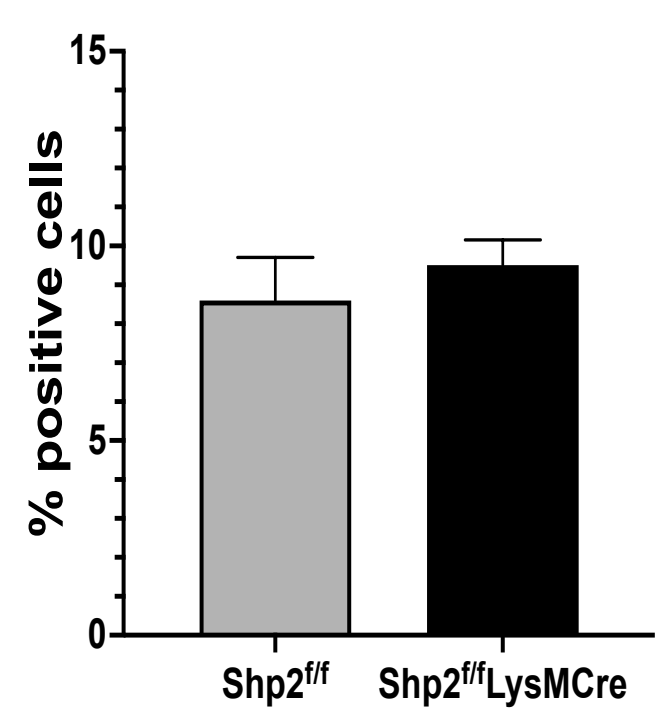

B

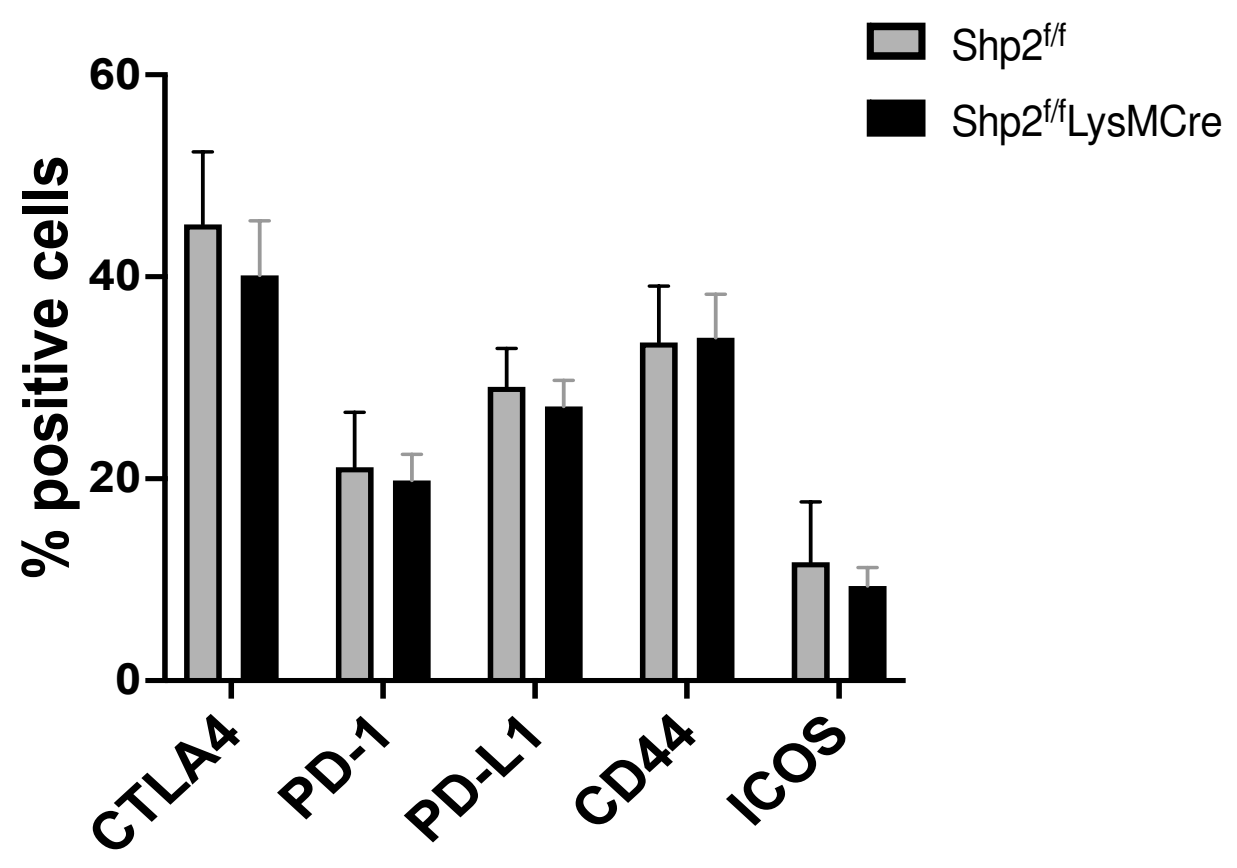

Fig. S6. Myeloid-specific SHP-2 deletion does not affect the expansion or activation of Treg cells. Tregs were identified by flow cytometry in TDLN of Shp2 ${ }^{\mathrm{f} / \mathrm{f}}$ and Shp2 ${ }^{\mathrm{f} / \mathrm{L}}$ LysMCre mice bearing MC17-51 tumors, (A). The expansion of the indicated markers on Treg cells was examined (B). Representative of one from five independent experiments with four to 6 mice per group. 


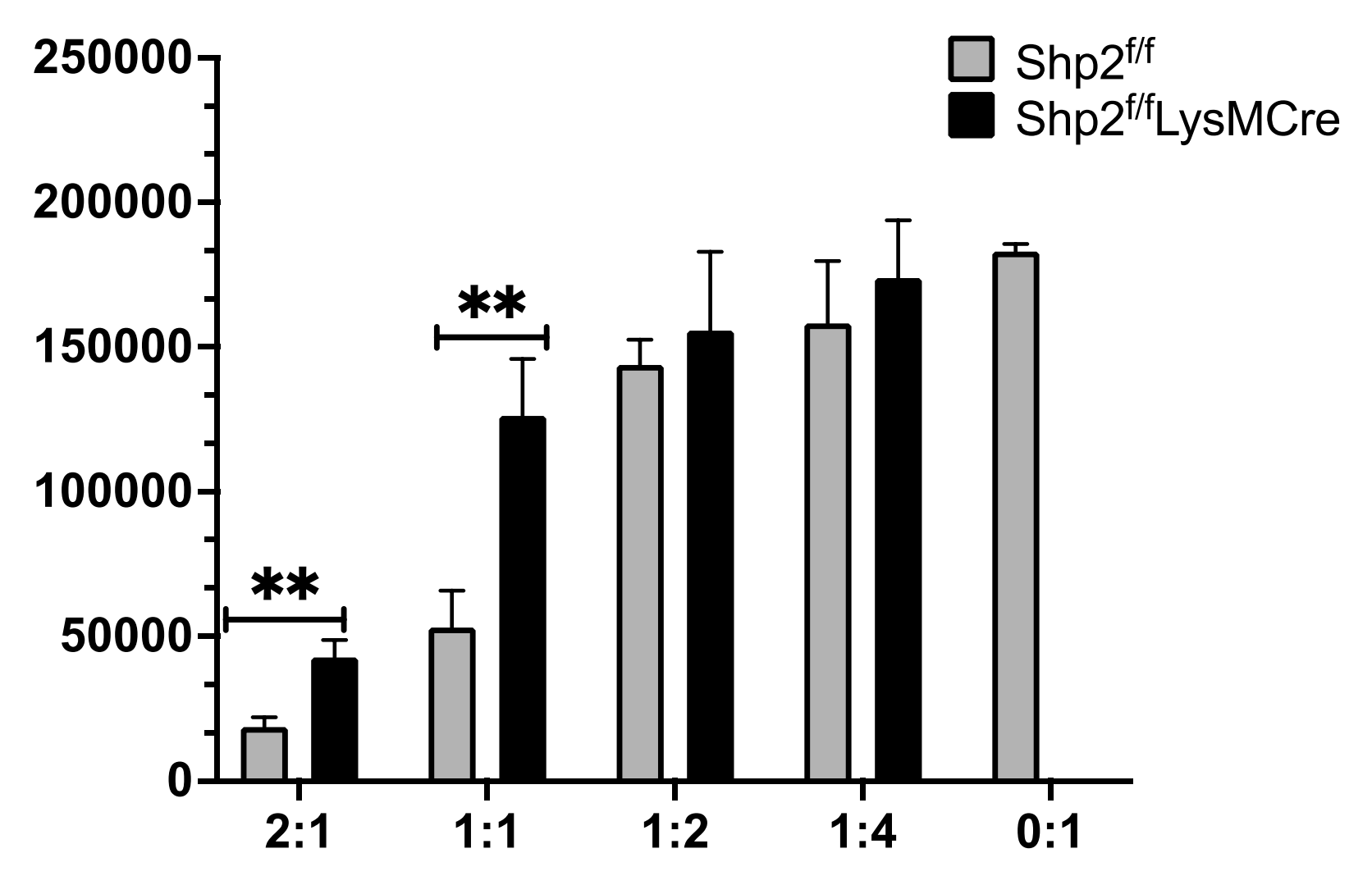

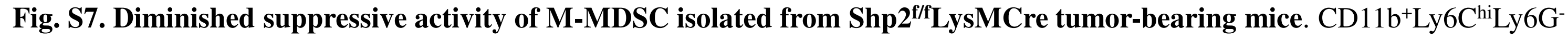
monocytic (M-MDSC) cells were isolated from Shp2 ${ }^{\mathrm{f} / \mathrm{f}}$ and Shp2 ${ }^{\mathrm{f} / \mathrm{L}} \mathrm{LysMCre}$ tumor-bearing mice and cultured at various ratios with OTI splenocytes $\left(1 \times 10^{5}\right.$ cells/well $)$ stimulated with $\mathrm{OVA}_{257-264}$. Mean $\pm \mathrm{SEM}$ of cpm values are shown. Results are representative of two separate experiments with $\mathrm{n}=7$ mice per group. 

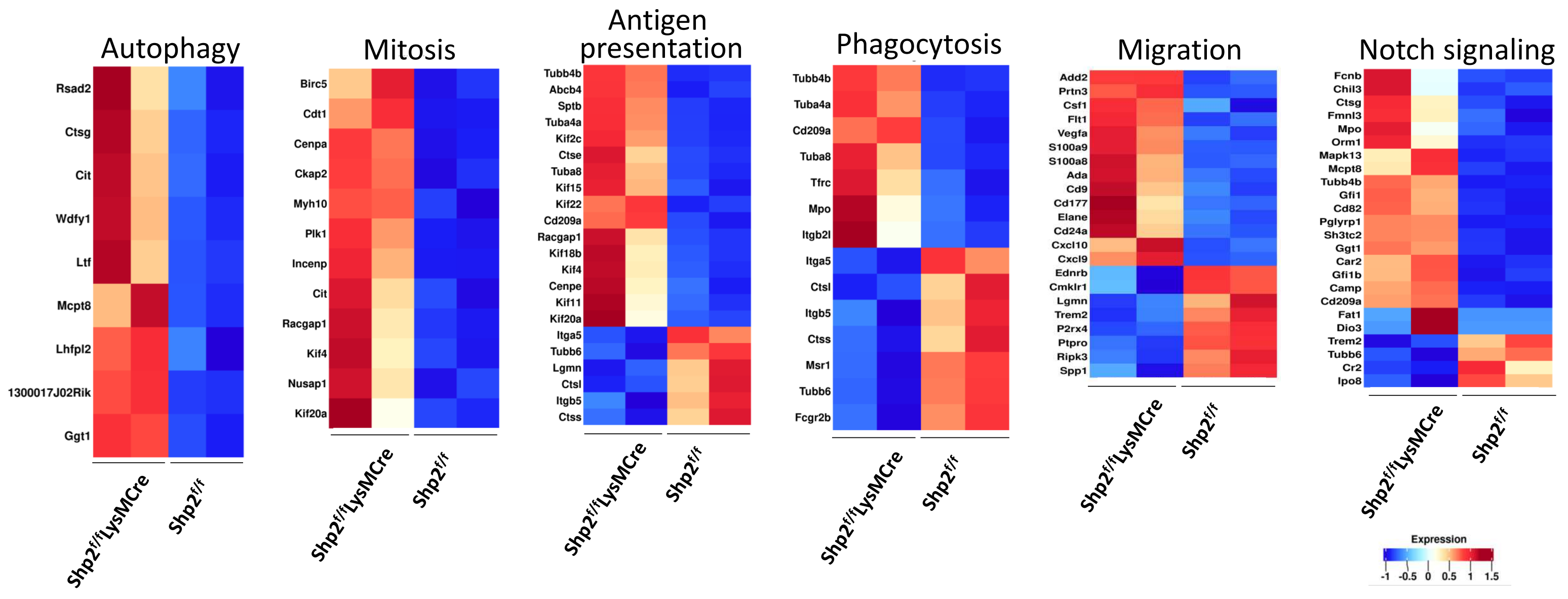

Fig. S8. Pathway enrichment analysis in PMN-MDSC. Pathway enrichment analysis was performed as described in Materials and Methods. Relative to those isolated from control tumor-bearing hosts, PMN-MDSC from Shp2 ${ }^{\mathrm{fl} / \mathrm{fl}}$ LysMCre tumor-bearing mice were enriched for processes involved in autophagy, mitosis, antigen presenting function, phagosome formation, leukocyte migration and Notch signaling. 


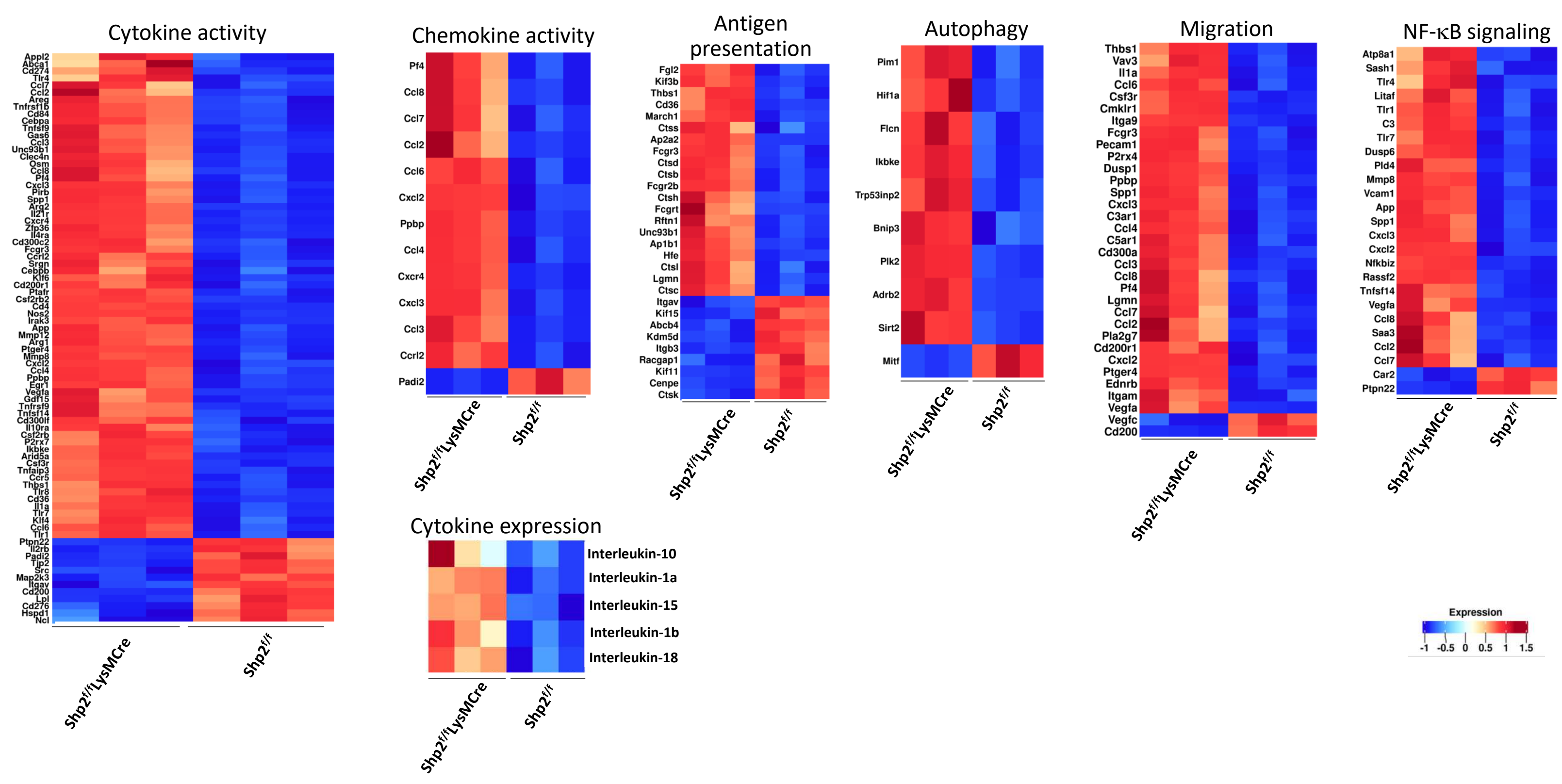

Fig. S9. Pathway enrichment analysis in TAMs. Pathway enrichment analysis was performed as described in Materials and Methods. SHP-2 deficient TAM display upregulation of gene programs related to cytokine and chemokine activity, cytokine expression, antigen presentation, autophagy, migration and $\mathrm{NF}-\mathrm{\kappa B}$ signaling. 
A

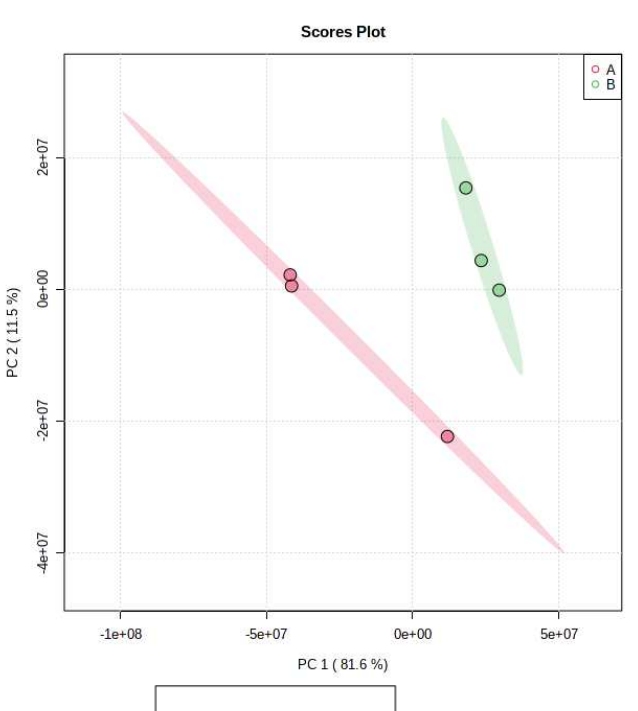

B

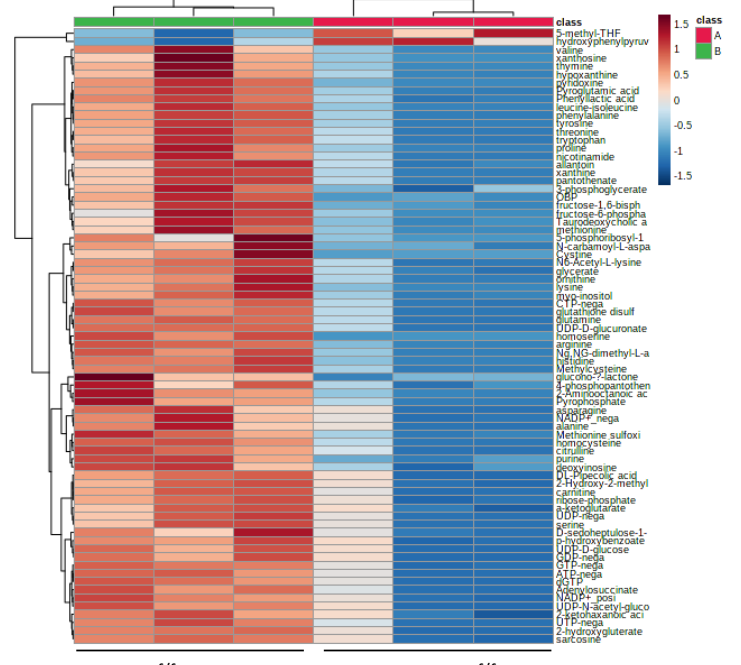

Shp2 $2^{\mathrm{f} / \mathrm{L} \text { LyMCre }}$

$\mathrm{Shp2}^{\mathrm{f} / \mathrm{f}}$

$={ }_{S H P 2} 2^{f / f}$
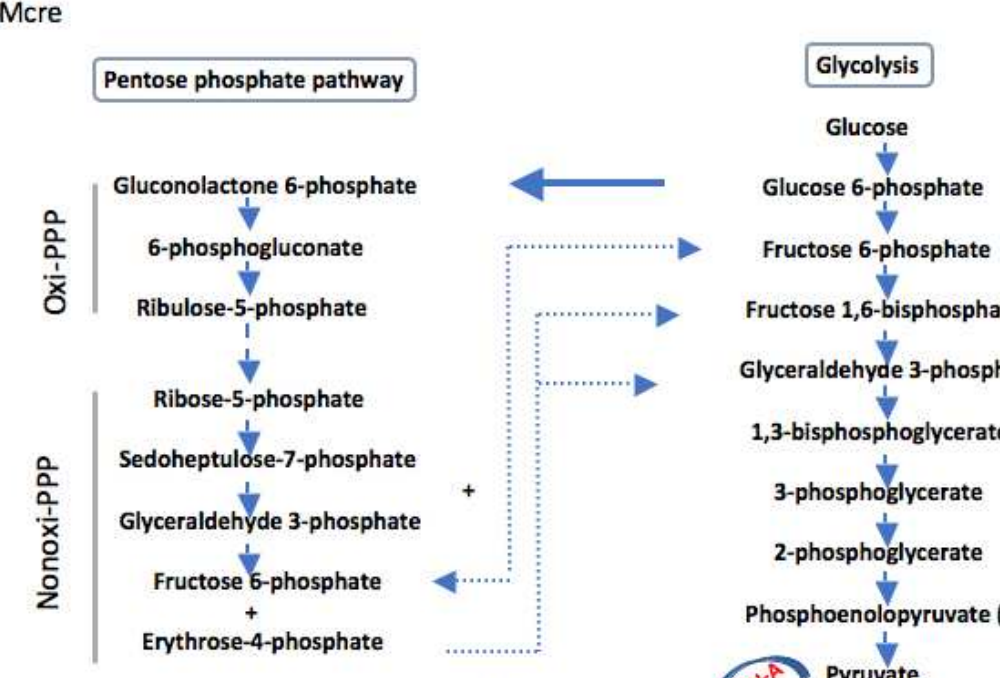

Fructose 1,6-bisphosphate

Glyceraldehyde 3-phosphate

1,3-bisphosphoglycerate

3-phosphoglycerate

2-phosphoglycerate

Phosphoenolopyruvate (PEP)

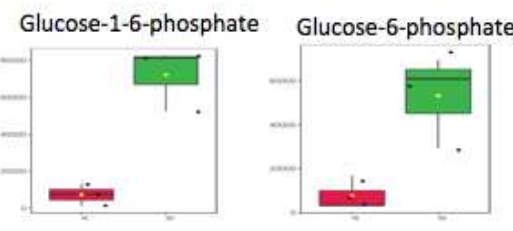

A-Ketoglutarate

A-Ketoglutarate

Succinte
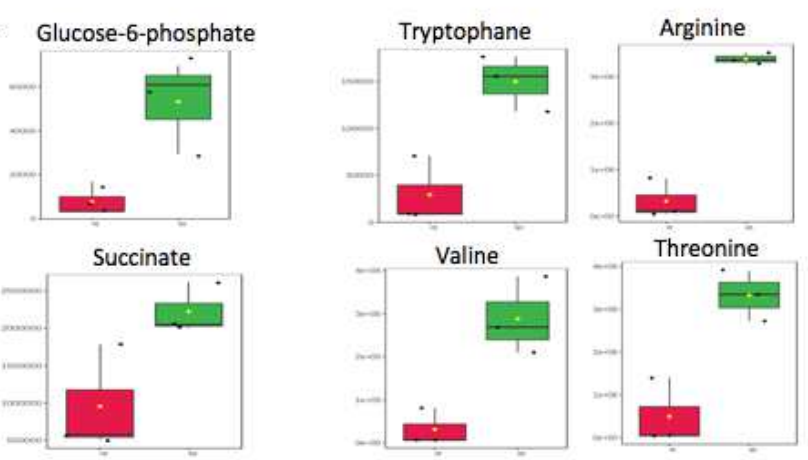

Valin
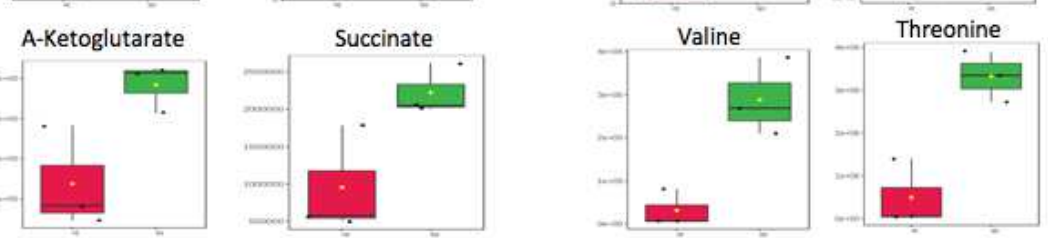

$\div$
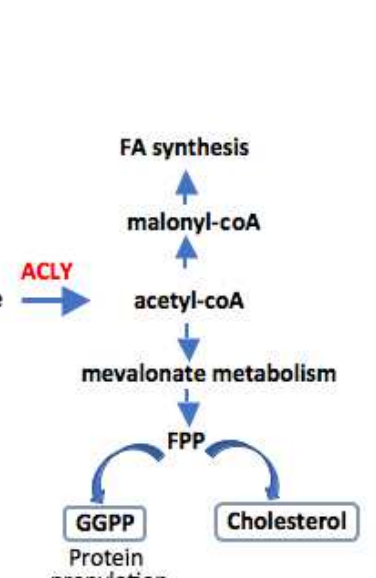

FA synthesis
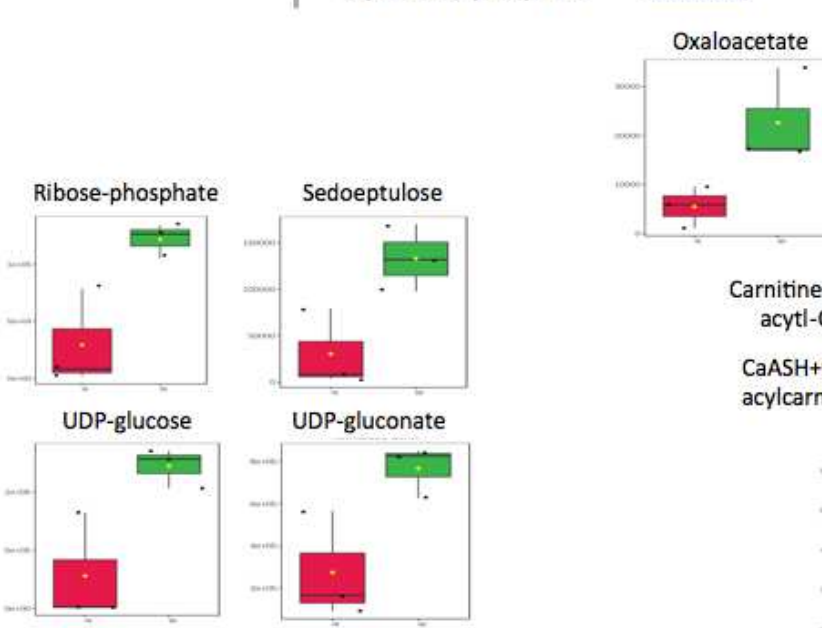

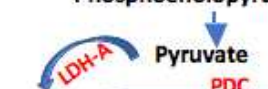
Lactate $\quad P D C$ cytoso

$$
\text { Oxaloacetate }
$$

Aconitate

aKetoglutarate

Malate TCACycle

Carnitine+fatty
acytl-CoA

CaASH+fatty Fumarate mitohondrion
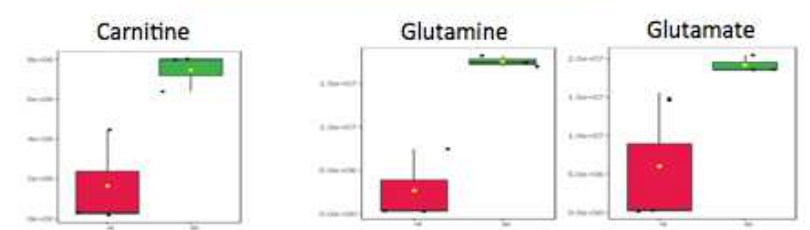

Protein
prenylation
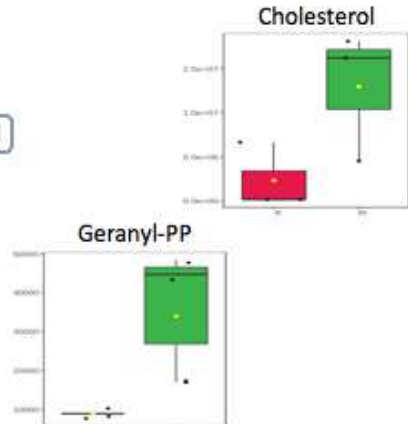

Fig. S10. Phagocytes generated from Shp2 ${ }^{\mathrm{f} / \mathrm{f}}$ LysMcre mice have enhanced metabolic activity. Lin ${ }^{\text {neg }}$ bone marrow cells from Shp2 ${ }^{\mathrm{f} / \mathrm{f}}$ and Shp2 ${ }^{\mathrm{f} / \mathrm{L}}$ LysMCre mice were cultured with G-CSF and GM-CSF for 48 hours and metabolite analysis was performed. (A) PCA (principal component analysis) showed distinct distribution of each group of samples, consistent with a different metabolic profile. (B) Unsupervised hierarchical clustering of top 75 metabolites is shown (log2FC $\geq 1$ ). (C)

Individual graphs of relative peak intensity of representative intermediate metabolites of glycolysis, PPP, TCA cycle in Shp2 ${ }^{\mathrm{f} / \mathrm{f}} \mathrm{LysMCre}$ and Shp2 $2^{\mathrm{f} / \mathrm{f}}$ mice. Results from one representative of two separate experiments are shown. 
TAMs

GO: Biological Process Pathways Enriched

Top 500 Up-regulated genes

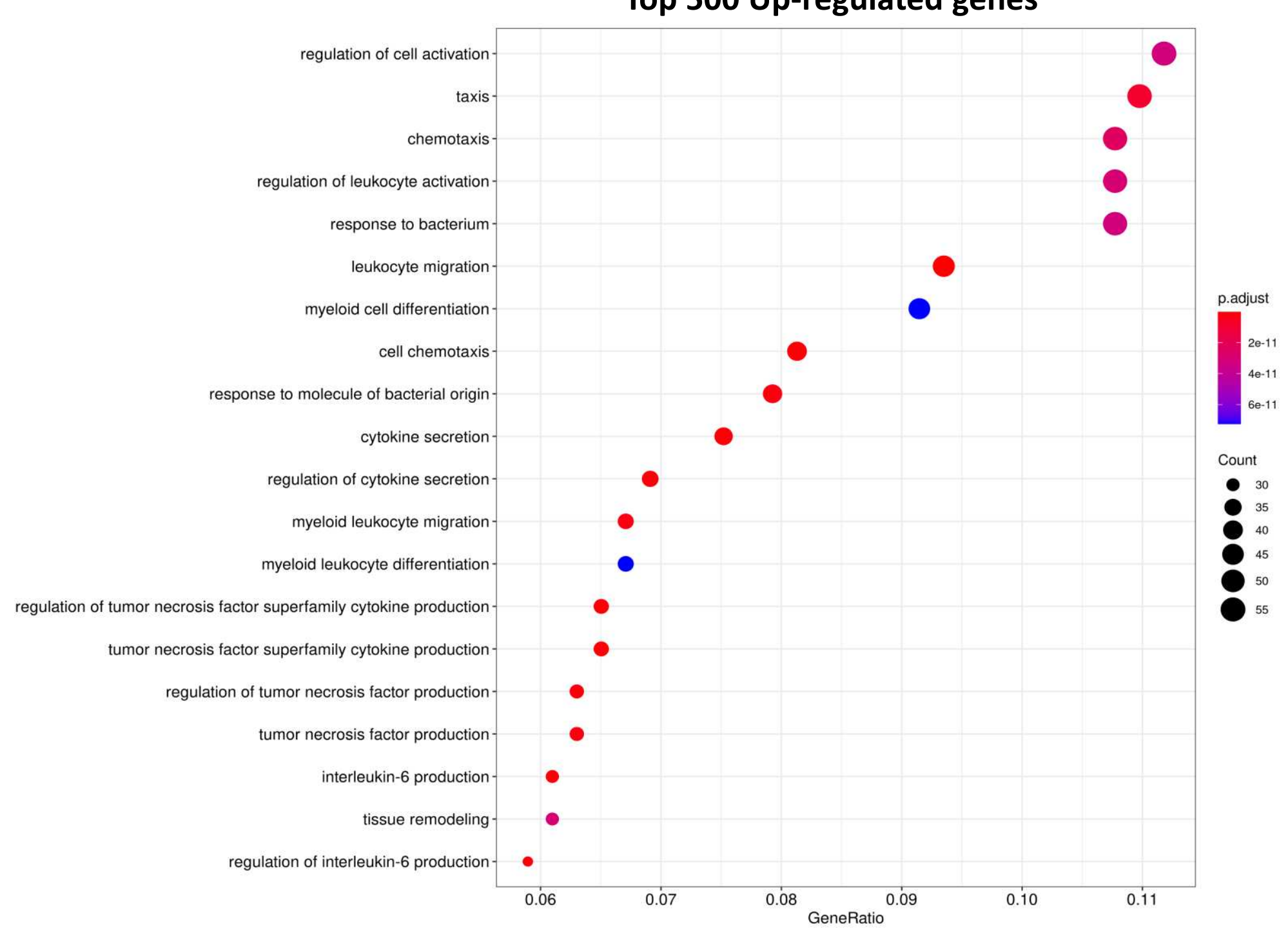

Fig. S11. Pathway enrichment analysis using Gene Ontology Biological Process Pathways gene set datasets showing top biological processed upregulated in TAMs from Shp2 $2^{\mathrm{f} / \mathrm{L}} \mathrm{LysMCre}$ mice. 
A

GM-CSF+IL-3 culture

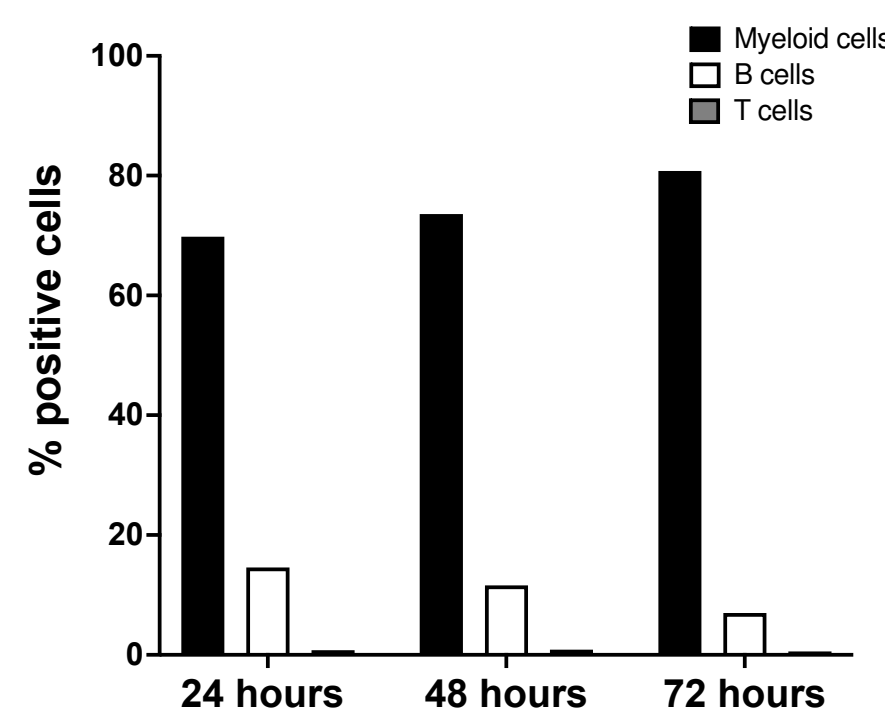

B

\section{PD-1}

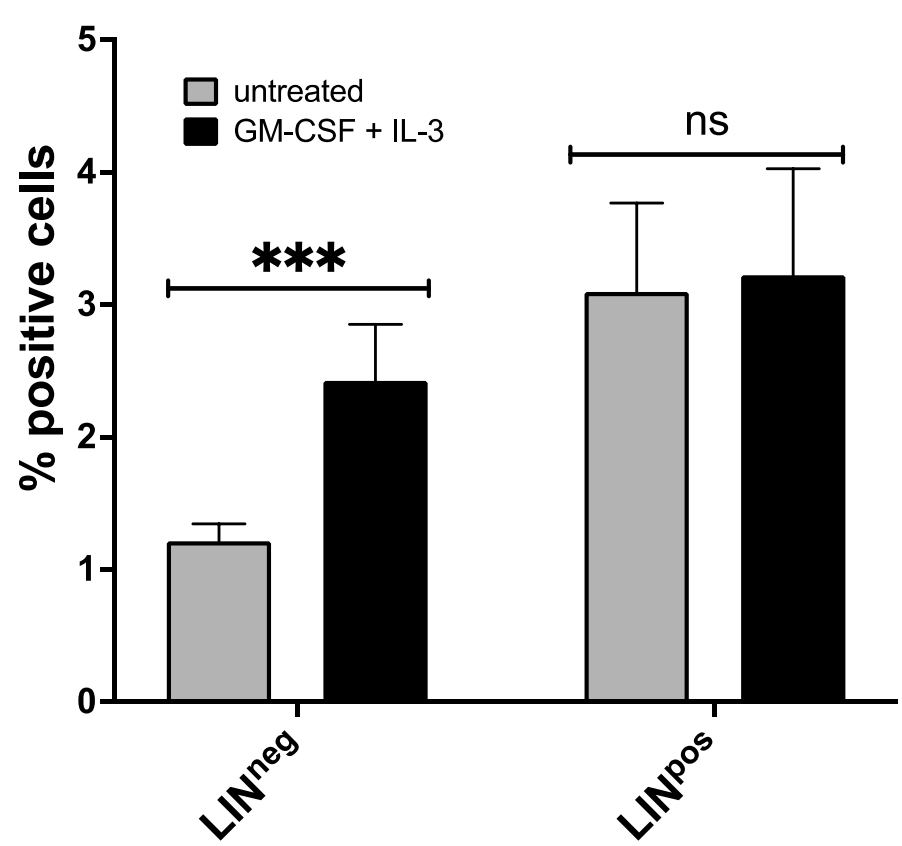

C

\section{PD-L1}

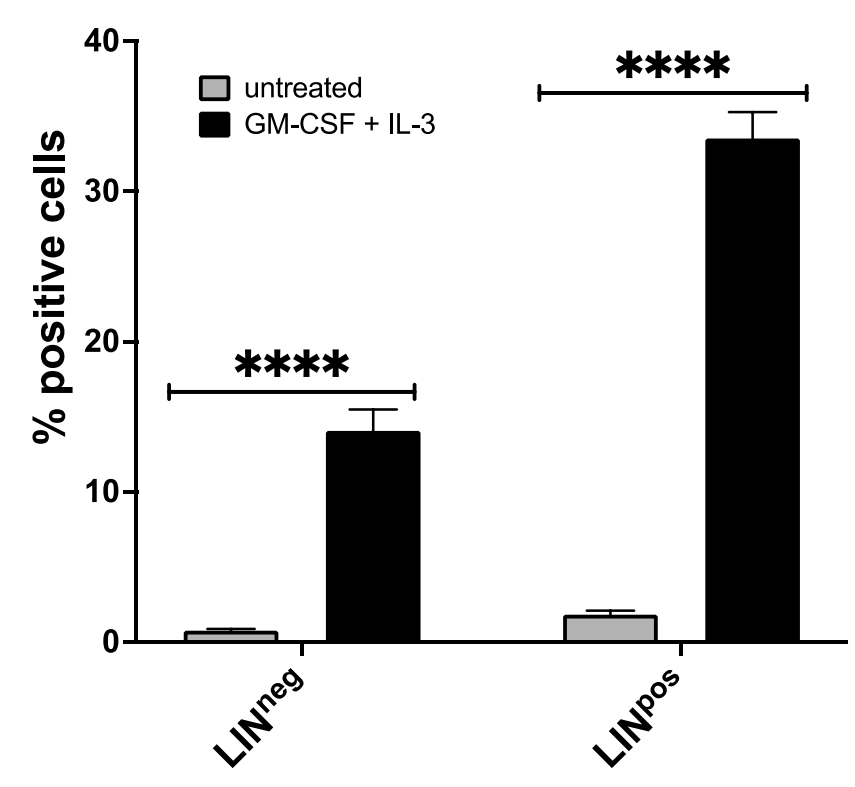

Fig. S12. Culture of bone marrow cells with GM-CSF+IL-3 induces upregulation of PD-1 and PD-L1 in Lin ${ }^{\text {neg }}$ and Lin ${ }^{\text {pos }}$ myelocytes. (A) Lin ${ }^{\text {neg }}$ bone marrow cells from WT C57BL/6 mice were culture with GM-CSF + IL-3 as described in Materials and Methods and the frequency of the indicated cell populations was assessed by flow cytometry. (B-C) Expression of PD-1 (B) and PDL-1 (C) in Lin ${ }^{\text {neg }}$ and Lin ${ }^{\text {pos }}$ subsets was examined by flow cytometry. $(* \mathrm{p}<0.05, * * p<$ $0.01, * * * \mathrm{p}<0.005, * * * * \mathrm{p}<0.001)$ 


\section{Supplementary Files}

This is a list of supplementary files associated with this preprint. Click to download.

- SupplementaryTable3.csv

- SupplementaryTable4.pdf

- SupplementaryTables1and2.xlsx

- SupplementraryDataset1GSEAMDSC.xIsx

- SupplementraryDataset2GSEATAMs.xIsx 